\begin{abstract}
Title of Dissertation: COLLECTIVE ENTITY RESOLUTION

IN RELATIONAL DATA

Indrajit Bhattacharya, Doctor of Philosophy, 2006

Dissertation directed by: Dr. Lise Getoor

Department of Computer Science
\end{abstract}

Many databases contain imprecise references to real-world entities. For example, a social-network database records names of people. But different people can go by the same name and there may be different observed names referring to the same person. The goal of entity resolution is to determine the mapping from database references to discovered real-world entities.

Traditional entity resolution approaches consider approximate matches between attributes of individual references, but this does not always work well. In many domains, such as social networks and academic circles, the underlying entities exhibit strong ties to each other, and as a result, their references often co-occur in the data. In this dissertation, I focus on the use of such co-occurrence relationships for jointly resolving entities. I refer to this problem as 'collective entity resolution'. First, I propose a relational clustering algorithm for iteratively discovering entities by clustering references taking into account the clusters of co-occurring references. Next, I propose a probabilistic generative model for collective resolution that finds hidden group structures among the entities and uses the latent groups as evidence 
for entity resolution. One of my contributions is an efficient unsupervised inference algorithm for this model using Gibbs Sampling techniques that discovers the most likely number of entities. Both of these approaches improve performance over attribute-only baselines in multiple real world and synthetic datasets. I also perform a theoretical analysis of how the structural properties of the data affect collective entity resolution and verify the predicted trends experimentally. In addition, I motivate the problem of query-time entity resolution. I propose an adaptive algorithm that uses collective resolution for answering queries by recursively exploring and resolving related references. This enables resolution at query-time, while preserving the performance benefits of collective resolution. Finally, as an application of entity resolution in the domain of natural language processing, I study the sense disambiguation problem and propose models for collective sense disambiguation using multiple languages that outperform other unsupervised approaches. 


\title{
COLLECTIVE ENTITY RESOLUTION IN RELATIONAL DATA
}

\author{
by \\ Indrajit Bhattacharya \\ Dissertation submitted to the Faculty of the Graduate School of the \\ University of Maryland, College Park in partial fulfillment \\ of the requirements for the degree of \\ Doctor of Philosophy \\ 2006
}

Advisory Committee:

Dr. Lise Getoor, Chair/Advisor

Dr. Carol Espy-Wilson, Dean's Representative

Dr. Amol Deshpande

Dr. Philip Resnik

Dr. Marie desJardins 
(c) Copyright by

Indrajit Bhattacharya

2006 
Dedication

To my parents. 


\section{Acknowledgments}

First and foremost, I would like to sincerely thank my advisor Lise Getoor for her help and support throughout my PhD experience. She gave me the opportunity to work on research problems that are relevant, challenging and interesting. But, more importantly, she introduced me to the world of research and has been a tutor in all the different aspects of it — from picking a problem to writing a paper. I have also learnt from her the importance of hard work and perseverance in the making of a successful researcher. My pursuit of a PhD has not always been smooth-sailing, and I would not have made it through, if it had not been for her patience and her support. The door to her office has been open for me whenever I needed to talk.

I would like to thank my other committee members, Philip Resnik, Amol Deshpande, Marie desJardins and Carol Espy-Wilson, for taking the time to review my dissertation and for their help and suggestions for improving it. Philip and Amol, in particular, have always been available for advice. I am thankful for their active help during the job-hunting process and for counseling regarding career options in general. I am also thankful for the opportunity to work with Yoshua Bengio during the early years of my PhD. For the KDD Entity Resolution Challenge in the summer of '05 and for the research on query-based entity resolution that it inspired, I worked in collaboration with my friend and fellow graduate student Louis Licamele. It has been a pleasure working with him. I could always count on his optimism when things looked bleak.

Among faculty members in the department, who have not been directly associated with my dissertation, I will always remember and be thankful for the many 
hours that Bobby Bhattacharjee spent with me discussing research, career options, and sometimes cricket to take a break from all those other things in life. I have also benefited immensely from my interactions with Hanan Samet and Amitabh Varshney. Among graduate students I have collaborated with, Srinivsan Parthasarathy introduced me to many interesting problems outside the domain of my dissertation. He was the main inspiration for our work on peer-to-peer systems, for which we also had Srinivas Kashyap in our team.

I have been fortunate to be a part of an excellent research group. I would like to sincerely thank all the LINQS members - Rezarta Islamaj, Prithviraj Sen, Mustafa Bilgic, Louis Licamele, Galileo Namata, Vivek Sehgal, Wontaek Tseo, John Park, Hyunmo Kang and Elena Zheleva - for providing a wonderful working atmosphere, from discussing research to reflecting on life over a cup of coffee. I would like to specially thank the fellow residents of 3228 A.V. Williams Building - Rezarta, Mustafa, Louis, Galileo, Vivek and also Nargess Memarsadeghi. It would have been lonely in there without them.

I have come to know several excellent people in the department during the course of my PhD. I will be always be thankful to Rajiv Gandhi for being a mentor during the early years. Among others, Gutemberg Guerra-Filho, Vijay Gopalakrishnan, Srinivasan Parthasarathy, Arun Vasan, Arunesh Mishra and Gaurav Aggarwal have always been good friends. When I needed company in A.V. Williams during weekends, I could count on Kaushik Mitra and Sandeep Manocha.

Several staff members in the department have been exceptionally helpful. Brenda Chick and Brad Plecs were always available for ready assistance, and Fatima 
Bangura and Felicia Chelliah will always be among the people I remember from my $\mathrm{PhD}$ experience.

As I have learnt over the last six years, there is much to the $\mathrm{PhD}$ experience beyond research, and the quality of life outside had a direct impact on the quality of research happening within the walls of the department. I have been fortunate to have several wonderful room-mates. Amit Roy-Chowdhury has been a proverbial friend, philosopher and guide. I owe a lot to Ayush Gupta and Supratik Datta, who have been my room-mates for the longest time. Beyond room-mates, Kaushik Chakraborty, Sharmistha Acharya and Suddhasattwa Ghosh have been friends through the highs and the lows.

Finally, there are those who can never be thanked enough, and it is useless to try. It would have been impossible to be through it all without the support of my parents. 


\section{Table of Contents}

List of Tables $\quad$ ix

List of Figures $\quad$ X

1 Introduction 1

1.1 Data Integration and Entity Resolution . . . . . . . . . . . . . . . . . 1

1.2 Collective Entity Resolution Using Relationships . . . . . . . . . . . . 4

1.3 Collective Relational Clustering . . . . . . . . . . . . . . . 6

1.4 Probabilistic Model for Collective Entity Resolution . . . . . . . . . . 8

1.5 Entity Resolution for Queries . . . . . . . . . . . . . . . . . 10

1.6 Applying Entity Resolution for Word Sense Disambiguation . . . . . 12

1.7 Terminology . . . . . . . . . . . . . . . . . 14

1.8 Specific Contributions and Organization of the Dissertation . . . . . . 16

2 Relational Clustering for Collective Entity Resolution 19

2.1 Motivating Example for Entity Resolution Using Relationships . . . . 19

2.2 Entity Resolution Using Relationships: Problem Formulation . . . . . 23

2.3 Entity Resolution Approaches . . . . . . . . . . . . . . . . 25

2.3.1 Attribute-based Entity Resolution . . . . . . . . . . . . 26

2.3.2 Naive Relational Entity Resolution . . . . . . . . . . . . . . . 26

2.3.3 Collective Relational Entity Resolution . . . . . . . . . . . . . 28

2.4 Neighborhood Similarity Measures for Collective Resolution . . . . . 31

2.4.1 Common Neighbors . . . . . . . . . . . . . . . . . . . . 31

2.4.2 Jaccard Coefficient . . . . . . . . . . . . . . . 32

2.4.3 Adamic/Adar Similarity . . . . . . . . . . . . . . . 33

2.4.4 Adar Similarity with Ambiguity Estimate . . . . . . . . . . . 34

2.4.5 Higher-Order Neighborhoods . . . . . . . . . . . . . . . 36

2.4.6 Negative Constraints From Relationships . . . . . . . . . . . . 37

2.5 Relational Clustering Algorithm . . . . . . . . . . . . . . . 38

2.5.1 Blocking to Find Potential Resolution Candidates . . . . . . . 38

2.5.2 Relational Bootstrapping . . . . . . . . . . . . . . . 40

2.5.3 Merging Clusters and Updating Similarities . . . . . . . . . 43

2.5.4 Complexity Analysis . . . . . . . . . . . . . . . . . . 44

2.6 Experimental Evaluation . . . . . . . . . . . . . . . 46

2.6.1 Evaluation on Bibliographic Data . . . . . . . . . . . . 46

2.6.1.1 Datasets . . . . . . . . . . . . . . 47

2.6.1.2 Evaluation ................ . . 49

2.6.1.3 Experimental Details . . . . . . . . . . . 50

2.6.1.4 Results .................. . 51

2.6.1.5 Execution Time . . . . . . . . . . . . . 58

2.6.2 Experiments on Synthetic Data ............ . 60

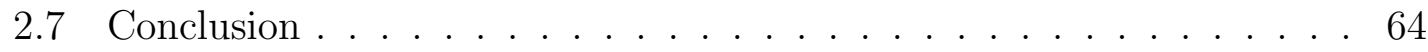


3 A Latent Dirichlet Model for Unsupervised Entity Resolution 66

3.1 A Motivating Example . . . . . . . . . . . . . . . 66

3.2 LDA Model for Authors . . . . . . . . . . . . . . . . . . 70

3.3 LDA Model for Author Resolution . . . . . . . . . . . . . . . . . . 72

3.4 Inference using Gibbs Sampling . . . . . . . . . . . . . . . . 74

3.5 Modeling Author Attributes . . . . . . . . . . . . . . . 76

3.6 Noise Model . . . . . . . . . . . . . . . . . . . . . . . . 77

3.7 Determining Number of Entities . . . . . . . . . . . . . . . . 78

3.7.1 Basic Inference With Gibbs Sampling . . . . . . . . . . . . . . 78

3.7.2 Relation to the Dirichlet Process . . . . . . . . . . . . . . . 79

3.7.3 Block Assignment for Entity Resolution . . . . . . . . . . . . 81

3.8 Determining Model Parameters . . . . . . . . . . . . . . . . 86

3.8.1 Number of Groups . . . . . . . . . . . . . . . . . 87

3.8.2 Hyper-parameters . . . . . . . . . . . . . . . . . . 87

3.8.3 Noise Model Parameters . . . . . . . . . . . . . . . . . . . . 88

3.9 Algorithm Refinements . . . . . . . . . . . . . . . . . . . 89

3.9.1 Bootstrapping Author Labels . . . . . . . . . . . . . . 89

3.9.2 Group Evidence for Author Self Loops . . . . . . . . . . . . . 89

3.10 Experimental Evaluation . . . . . . . . . . . . . . . 90

3.10 .1 Results on Citation Data . . . . . . . . . . . . . . . . . . 90

3.10.2 Properties of Collaborative Graphs . . . . . . . . . . . . . . . 95

3.10.3 Comparison With Collective Relational Clustering . . . . . . . 98

3.11 Conclusions . . . . . . . . . . . . . . . . . . . . . . . . 99

4 Entity Resolution for Queries 101

4.1 Motivativation for Entity Resolution Queries . . . . . . . . . . . . . 101

4.2 Entity Resolution Queries: Formulation . . . . . . . . . . . . . . . . 104

4.3 Performance Dependencies in Relational Clustering . . . . . . . . . . 105

4.3.1 Performance Analysis of Attribute-based Resolution . . . . . . 107

4.3.2 Characterizing Relations . . . . . . . . . . . . . . . 108

4.4 Two-Stage Query Processing . . . . . . . . . . . . . . . . . . . . . 112

4.5 Adaptive Query Expansion . . . . . . . . . . . . . . . . 117

4.6 Experimental Results . . . . . . . . . . . . . . . . . . 119

4.6.1 Experiments on Real Data . . . . . . . . . . . . . . . . 119

4.6.2 Experiments using Synthetic Data. . . . . . . . . . . . 127

4.7 Conclusions . . . . . . . . . . . . . . . . . . . . . 129

5 Word Sense Disambiguation Using Bilingual Probabilistic Models 131

5.1 Word Sense Disambiguation: Introduction and Related Work . . . . . 131

5.2 Probabilistic Models for Parallel Corpora . . . . . . . . . . . . 135

5.2.1 Notation . . . . . . . . . . . . . . . . . . . 135

5.2 .2 The Sense Model . . . . . . . . . . . . . . . . . . 136

5.2 .3 The Concept Model . . . . . . . . . . . . . . 137

5.3 Constructing the Senses and Concepts . . . . . . . . . . . 138

5.3.1 Building the Sense Model . . . . . . . . . . . . . . 139 
5.3.2 Building the Concept Model . . . . . . . . . . . . . . . . . . 140

5.4 Learning the Model Parameters . . . . . . . . . . . . . . . . . . . . . 142

5.4.1 EM for the Sense Model . . . . . . . . . . . . . . . . . . . . . 142

5.4 .2 EM for the Concept Model . . . . . . . . . . . . . . . . . 143

5.4.3 Initialization of Model Probabilities . . . . . . . . . . . . 143

5.5 Experimental Evaluation . . . . . . . . . . . . . . 144

5.5.1 Evaluation with Senseval Data . . . . . . . . . . . . . 145

5.5.2 Semantic Grouping of Spanish Senses . . . . . . . . . . . . . . 147

5.6 Model Analysis . . . . . . . . . . . . . . . . . . . . 149

6 Related Work 152

6.1 Approximate Matching . . . . . . . . . . . . . . . . . . . 152

6.2 Theoretical Bounds for Cleaning . . . . . . . . . . . . . . . 153

6.3 Efficiency Issues . . . . . . . . . . . . . . . . . . . . . . . . . . . . . . . . . . . . . . . . . . . . . .

6.4 Probabilistic Models for Entity Resolution . . . . . . . . . . . . . . 155

6.5 Non-probabilistic Relational Approaches . . . . . . . . . . . . . . 157

6.6 Group and Topic Modeling . . . . . . . . . . . . . . . . . 159

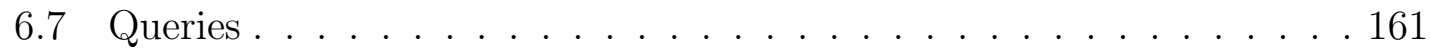

6.8 Data Cleaning Tools . . . . . . . . . . . . . . . . . . 161

6.9 Application Domains . . . . . . . . . . . . . . . . . . . . 162

6.10 Evaluation Metrics . . . . . . . . . . . . . . . 162

7 Conclusions and Future Directions 164

A Synthetic Data Generation 169

$\begin{array}{ll}\text { Bibliography } & 175\end{array}$ 


\section{List of Tables}

2.1 Performance of different algorithms on real datasets . . . . . . . . . 51

2.2 Performance of neighborhood sim. measures on real datasets . . . . . 56

2.3 Execution times of different algorithms . . . . . . . . . . . . . 59

3.1 Performance of baselines using SoftTF-IDF . . . . . . . . . . . . 93

3.2 Performance of LDA-ER on real datasets . . . . . . . . . . . . . . . . 94

3.3 Performance of LDA-ER over varying number of groups . . . . . . . . 95

3.4 Comparison of LDA-ER with relational clustering . . . . . . . . . . . 98

4.1 Resolution accuracy for queries using different algorithms . . . . . . . 123

4.2 Resolution accuracy using different adaptive expansion strategies . . . 125

4.3 Comparison between unconstrained and adaptive expansion . . . . 126

5.1 Comparison with a baseline WSD model . . . . . . . . . . 146

5.2 Examples of Spanish concepts and senses . . . . . . . . . . . . . . 148 


\section{List of Figures}

2.1 References in the bibliographic example . . . . . . . . . . . . . 20

2.2 Example of (a) reference graph and (b) entity graph . . . . . . . . . 22

2.3 Abstract representation of (a) reference graph and (b) entity graph . 24

2.4 The relational clustering algorithm . . . . . . . . . . . . . 39

2.5 Resolution performance over varying $\alpha$ on real datasets . . . . . . . . 54

2.6 Comparison of different relational similarity measures . . . . . . . . 57

2.7 Execution time of $\mathrm{CR}$ with increasing references . . . . . . . . 60

2.8 Comparison of performance on synthetically generated data . . . . . 61

3.1 Illustration of author entities and collaboration groups . . . . . . 68

3.2 Plate representation for (a) author model and (b) reference model . . 71

3.3 Comparison of LDA-ER with baselines on synthetic data . . . . . . 96

4.1 Illustration of (a) identifying relation and (b) ambiguous relation . . . 109

4.2 Construction of relevant set for a query . . . . . . . . . . . . . 114

4.3 Growth of relevant set size and query processing time . . . . . . . 120

4.4 Effect of identifying and ambiguous relations . . . . . . . . . 127

4.5 Effect of using increasing levels of co-occurrence . . . . . . . . . . 128

5.1 Graphical representations of Sense and Concept models . . . . . . . 136

5.2 Examples of Sense and Concept Models . . . . . . . . . . . . . 139

5.3 Comparison with Senseval2 WSD Systems . . . . . . . . . 147

A.1 The synthetic data generation algorithm . . . . . . . . . . . 171 


\section{Chapter 1}

\section{Introduction}

\subsection{Data Integration and Entity Resolution}

The phenomenal expansion of the world wide web, improved acquisition technology and increasing affordability of storage media have all contributed to an explosive growth in the volume of publicly accessible data in digital form. This immense volume of data, which was unimaginable a decade ago, brings with it new possibilities for automated reasoning and knowledge discovery. As an illustration of how large volumes of data can help, there have recent news reports of doctors using web search technology for diagnosing complex symptoms in patients [84]. In this study, doctors searched using patient symptoms without knowing the right diagnosis and then selected the most relevant diagnosis from the top three results. This returned the correct diagnosis for more than $50 \%$ of the patients studied. One of the biggest hurdles for analyzing available data is that information is most often dispersed over several data sources. It is often possible to make use of all the available data most effectively if it is acquired (or indexed) and integrated into a central repository. There are several information repositories, such as CiteSeer for computer science publications, that automatically acquire data from different information sources us- 
ing improved crawling technologies. However integration of the acquired data into a consistent and coherent form is a more challenging problem. In the medical diagnosis example, the doctors had to manually find the most relevant match for each query, but completely manual curation is impossible in all but the smallest databases. As a result, alongside automated acquisition, there has been an increasing dependence on automated techniques for integrating the data and for maintaining quality of information. While we have seen a surge in research interest in this area over the last decade, the problems are expectedly quite daunting. Accuracy being critical in many applications, there is need for further research in this area.

Entity resolution is an important component of data integration that comes up frequently. In many databases, records refer to real-world entities, and as such databases grow, there can many different records that refer to the same entity. For example, a social network database can have different records with names 'J. Doe', 'Jonathan Doe' and 'Jon Doe' that refer to the same person. In the absence of keys such as social security numbers, this duplication issue $[52,78]$ leads to several different problems, such as redundant records, data inconsistencies, incorrectness of computed statistics, and many others. From a knowledge discovery perspective, mining data that has unresolved duplicates is very likely to yield patterns that are both inaccurate and incomplete. This issue also comes up when integrating data from different heterogeneous sources without shared keys and sometimes different schemas as well [28]. Broadly, I call such database records references to real world entities, and the entity resolution problem involves (a) finding the underlying entities in the domain and (b) tagging the references in the database with the entities 
to which they correspond. Most often, these two problems cannot be solved independently and need to be addressed at the same time.

In addition to databases, entity resolution is a common problem that comes in different guises (and is given different names) in other computer science domains. Examples include computer vision, where we need to figure out when features in two different images refer to the same underlying object (the correspondence problem); natural language processing where we would like to determine which noun phrases refer to the same underlying entity (co-reference resolution); data fusion or conflation in the geospatial domain, where spatial entities from different maps or images need to be matched. The problem goes by different names even inside the database community. Deduplication $[52,78]$ refers to problem of determining which records or tuples within the same database or relational table correspond to the same real world object. For data integration, approximate joins [28] are used for consolidating information from multiple sources.

Entity resolution is a difficult problem and cannot be solved using exact matches on tuple attributes, due to two main reasons that we discuss in greater detail in Section 2.1. First, there is the identification problem, when different representations arising from recording errors or abbreviations refer to the same entity. In the earlier example, figuring out that 'Jon Doe' and 'Jonathan Doe' are the same person is an instance of this problem. Failure in identification affects recall. The second issue is disambiguation. It is possible that two records with name 'J. Doe', the same address and the same age refer to two brothers and not to the same person. This affects precision. 
As I discuss in more detail in the chapter on related work (Chapter 6), the study of entity resolution goes back a long way to Newcombe et al. [83] who introduced the record linkage problem and Fellegi and Sunter [44] who formalized it. Early approaches to entity resolution prescribed fuzzy attribute matches and many sophisticated techniques have since been developed. However, approaches based solely on attributes still cannot satisfactorily deal with the problem of false attribute matches and, in general, it may be hard to improve precision and recall at the same time using just attributes of individual records. The problem is compounded by the lack of readily available training data. Preparing ground truth by manually tagging pairs of references as matches or non-matches can be very painstaking, and typically, labeled samples are sparse for most domains, if available at all. To get around this issue, the focus has often been on developing unsupervised approaches for resolving entities.

\subsection{Collective Entity Resolution Using Relationships}

In many domains, there may be additional information that we can use for resolving entities. The underlying entities often exhibit strong relational ties to certain other entities. For instance, in social networks, people interact frequently with their close friends, while in academic circles, researchers collaborate frequently with their close associates. When such ties exist between entities, co-occurrences between the references to these entities can be observed in the data. Names of colleagues co-occur as author names in publications and names of friends are often found to co-occur in 
emails. In the social network example, we may have records showing that 'J. Doe' communicates most with 'D. Smith' while 'Jon Doe' has frequent communications with 'Don Smith'. The goal is to make use of such relationships between references to improve entity resolution performance. One way is to use the attributes of related records as well when computing fuzzy matches. To compare the 'J. Doe' and 'Jon Doe' references, we may also compare the names of their associates. While this may lead to an improvement, it will not always work. For example, we do not want to merge two person records simply because their friends have similar names.

The correct evidence to use for the 'J. Doe' and 'Jon Doe' references is whether their best friends are in fact the same entity. This implies that in order to decide about one reference, we need to know about the entities for some other references. However, the problem is that we do not know the entities for these related records either. So how can we use these relations then? I use the idea of collective entity resolution, where the entities for related references need to be determined jointly rather than independently.

Collective entity resolution using relations is a challenging problem for two main reasons. This problem is an instance of collective clustering, where cluster membership for any reference depends on cluster memberships of all related references. Collective classification has been studied extensively in the machine learning literature in recent years $[25,85,62,48,64,99]$. But little work has been done for collective clustering using relationships. In order for distance or similarity based approaches to work for this problem, the relationships need to be incorporated into the similarity / distance measure. On the other hand, for model-based clustering to be 
used, we need to come up with representations for relationships between the underlying entities or clusters. Aside from the modeling issues, the other huge challenge is in terms of computation. The different clusters or entities cannot be determined independently, but instead the space of joint cluster assignments for related references needs to be explored. In contrast to attribute-based resolution, the database cannot be cleaned with a single-pass approach anymore. It is necessary to resort to iterative approaches, where each resolution that we make potentially provides new evidence for determining the entities for other related references. Efficiency in handling these dependencies is a key concern in designing algorithms for this problem. The challenges for collective clustering are daunting, but there is the promise that resolution accuracy can be significantly improved over traditional techniques.

In this dissertation, I first address the problem of collective entity resolution using relationships and propose two novel approaches for solving it. The first is a greedy agglomerative clustering approach called collective relational clustering $[8,7,9,11,10]$ and the second is a probabilistic generative model which I call $L D A-E R[12,10]$.

\subsection{Collective Relational Clustering}

In essence, collective relational clustering is a hierarchical clustering algorithm, where I start from an initial cluster assignment and then proceed by merging pairs of clusters. This is similar to greedy agglomerative clustering with a key difference. The similarity measure for cluster pairs accounts for relationships between different 
references, and as a result of this, each merge operation affects similarities for related cluster pairs. In the social network example, suppose 'J. Doe' is known to be friends with 'D. Smith' and 'K. Zabrinsky', while 'Jon Doe' has 'Don Smith' and 'Kate Zabrinsky' as friends. Then, if the relational clustering algorithm assigns the two Zabrinsky references to the same cluster in some iteration, that increases the similarity for the two 'Smith's and for the two 'Doe's, since they are now found to be friends with the same person. The merge operations continue until the similarity between the closest cluster pair drops below some threshold. The cluster similarity measure combines attribute similarity of the clusters with their relational similarity. The relational similarity is determined by the neighborhood of each cluster, and I explore different ways for measuring shared neighborhood between clusters. In parallel, I address the computational challenge arising from the dependencies by efficiently finding the most similar cluster pair and updating similarities in each iteration using novel indexing mechanisms.

The relational clustering algorithm has many attractive features. First, it is simple to understand and the incremental evidence is easy to interpret. It is easily customizable for specific domains by picking the best attribute similarity measures for that domain. Due to the monotonicity of the process where clusters always merge and also to the efficient implementation, it is quite fast. At the same time it works very well in practice. However, it has a few short-comings as well. First, as with traditional agglomerative clustering approaches, a similarity threshold needs to be specified as a termination condition. Secondly, clusters are only merged in this algorithm. Two clusters once merged can never split back to account for evidence 
that might be found in some later iteration. This speeds up the algorithm but also restricts the space of joint cluster assignments that it can explore. Also, different merge sequences can lead to different resolution results. The second approach that I propose is designed to deal with these specific issues.

\subsection{Probabilistic Model for Collective Entity Resolution}

The second approach that I propose for collective entity resolution using relationships is a non-parametric probabilistic model. It is a probabilistic generative model that describes how references for related entities might co-occur in the data. It represents relationships between underlying entities using the novel idea of groups of entities. References to entities that are members of the same group are more likely to co-occur in the data. In the social network example, this captures the notion of circles of friends. If the person entities 'Jonathan Doe', 'Donald Smith' and 'Katherine Zabrinsky' are members of the same group of friends, then they are expected to participate in conversations more frequently. This is similar to the idea of Latent Dirichlet Allocation or LDA that is used for topic mixtures in document modeling - hence the name $L D A-E R$. The critical difference with LDA is that the entities are not observed directly. Instead, only the references to entities, for example the names of the people, are observed in the data. The entities need to be determined using the additional group evidence that is discovered from the co-occurrences.

The next challenge for the $L D A-E R$ model is the design of tractable inference algorithms. As in the LDA model, exactly inferring the groups and entities for the 
observed references is intractable for $L D A$ - $E R$. I propose an approximate inference strategy based on Gibbs Sampling that infers the most likely group and entity for each reference. Additionally, it automatically discovers the most likely number of entities for the references without requiring any user specified threshold to be specified. This overcomes the first short-coming of the relational clustering approach, namely that of threshold selection. In order to address the second issue - that of clusters only being allowed to merge - I propose a sampling strategy for inference, where a reference may be assigned to a new entity at any iteration of the algorithm depending of current evidence. I also improve the computational complexity of the inference algorithm by proposing an improved merge-split sampling strategy where entities make random decisions to merge or split depending on available evidence. In addition to non-parametric resolution of the references, as an interesting byproduct, the model returns hidden group structures among the underlying entities that provide additional structural insight about the domain. However, these benefits of $L D A$-ER come at a price - added computational overhead. Since it does not take the greedy route of merging the closest clusters, but instead looks at all the entities, each iteration is more expensive. Additionally, it is not possible to set a worst-case upper-bound for the number of iterations, as with a greedy approach.

In summary, both of the proposed approaches for collective entity resolution have their advantages and disadvantages. For domains where quick results are necessary and termination thresholds can somehow be determined, the relational clustering approach should be preferable. In other cases, where specifying a termination threshold or an approximate number of entities apriori is difficult, $L D A-E R$ should 
be more useful. Also, in domains such as collaboration and social networks, where discovery of hidden group structures is relevant, $L D A-E R$ may be the preferred approach.

\subsection{Entity Resolution for Queries}

The resolution approaches that I propose in the first part of this dissertation are collective in nature - they resolve the references for an entire database as a whole. This works well for offline cleaning, but is not very useful for processing queries. Users query the web and different online databases everyday, and expect to get answers that are entity resolved, either directly or indirectly. For example, we may query the CiteSeer database of computer science publications looking for books by 'S Russell'. This query would be easy to answer if all author names in CiteSeer were correctly mapped to their entities. But, unfortunately, this is not the case. Going by CiteSeer records, Stuart Russell and Peter Norvig have written more than 100 different books together [86]. Alternatively, in our social network example, we may be searching different social network communities for a person named 'Jon Doe'. In this case, each online community may individually have records that are clean. But query results that return records from all of them together may have unresolved entities. Additionally, in both cases, it is not sufficient to simply return

records that match the query name, 'S. Russell' or 'Jon Doe' exactly. We need to retrieve records with similar names as well, but, more importantly, partition the records that are returned according to their entities. 
In this thesis, I motivate the problem of query-time entity resolution and apply collective resolution techniques for the problem of answering queries [13]. The biggest issue with this approach is the dependency structure of collective resolution. In order to reason about the query records, it is necessary to reason about their related records, which in turn require reasoning about their related records and so on. I first formally analyze how accuracies for resolving different entities depend on each other and on different structural characteristics of the data as a result of collective resolution. Then I propose a two stage strategy for localizing collective resolution. First, the relevant records necessary for answering the query are extracted by a recursive expansion process and then collective resolution is performed on the extracted records only. Using formal analysis, I show that the recursive expansion process can be terminated at reasonably small depths for accurately answering any query; the returns fall off exponentially as neighbors that are further away are considered. However, the problem with this unconstrained expansion process is that it may return too many records even at small depths that are impossible to resolve at query time. I address this issue using an adaptive strategy that only considers the most informative of the related records for answering any query. This significantly reduces the number of records that need to be investigated at query time, but, most importantly, does not compromise on the resolution accuracy for the query. In summary, the adaptive expansion strategy enables query-time resolution while preserving the performance benefits of collective resolution. 


\subsection{Applying Entity Resolution for Word Sense Disambiguation}

As an application of entity resolution in the domain of natural language processing, I consider the problem of word sense disambiguation and investigate how collective entity resolution using relationships can be useful for this problem [14]. Words in natural language documents are often ambiguous in terms of their senses. The identification and disambiguation issues that I mentioned in the context of entity resolution are well studied in the area of linguistics as well. Identification is necessary for the problem of synonymy, where different words can be used to refer to the same sense. On the other hand, we also have polysemous words that can correspond to multiple senses. Sense disambiguation deals with this second aspect of the problem. Consider the word 'bank' in English. According to the WordNet sense hierarchy, this word has 10 possible senses - financial institution, shore and reserve/stockpile being the three most common ones. Given two different occurrences of the word 'bank' in a natural language corpus, we need to decide whether they refer to the same sense or to different senses. This version of the problem is usually referred to as sense discrimination [95]. For the sense disambiguation problem, sense definitions are used from available sense hierarchies such as WordNet and then each occurrence of an ambiguous word needs to be tagged with one of its possible senses.

Traditional approaches to sense disambiguation make use of the context around a word. For example, the occurrence of 'breeze', 'sand' or 'water' is very likely to suggest the shore sense of bank and 'transaction' would suggest the financial institution sense. This is very similar to using attributes of a specific reference 
to determine the corresponding entity. More recent approaches make use of cooccurrence relationships in the form of translations. It is known that translations can help disambiguate senses $[22,33,34,55,91]$. For example, when 'bank' is translated in Spanish as 'orilla', it most likely to mean shore. Following Diab and Resnik [38], I make use of parallel corpora where the same document is available in multiple languages, for example in English and French as in the Canadian Hansards. Then aligned translation threads spanning documents in multiple languages serve as co-occurrence relations that we can use for resolving senses. As for entity resolution, I explore the problem of collective sense disambiguation, where senses are resolved for multiple languages simultaneously.

Despite the striking similarities with the entity resolution problem, the word sense disambiguation problem has certain interesting features that set it apart. First, we can make use of the sense definitions available for English words from the WordNet hierarchy. Effectively, these provide us with the domain entities for English words and we do not need to discover them. While this simplifies part of the problem, certain other aspects make it more challenging. Each translation thread spans words from multiple languages, each of which has its own defined senses. In essence, we can imagine this as an instance of multi-type entity resolution, where the co-occurrence relations connect entities of multiple types, each of which can be ambiguous. The other interesting aspect is the availability of the WordNet ontology for English (and more recently some other languages as well). On one hand, this provides sense definitions for words. On the other, it opens up possibilities for resolution approaches that can make use of the information-rich hierarchy, for instance 
in defining similarity measures between senses [89]. I propose two different probabilistic generative models for collective sense disambiguation from translations. The approach that I propose makes use of the WordNet structure to resolve senses in English and additionally to construct a semantic hierarchy for any secondary language for which translations are available.

\subsection{Terminology}

Before moving on to the main chapters of the dissertation, I review the terminology that I have established in this introductory chapter and will be using in the rest of the dissertation:

- Entity: An entity is a real world object, such as a person, place, organization, event, etc. that is easily recognized by a human being. Entities can also be abstract, such as a sense in the context of linguistics. The entities may be known for some domains. In others, they need to be discovered.

- Reference: A reference or a record is an observation or a mention of an entity, such as names of persons or places. A tuple in a census database is an example of a reference. In many cases, references need to be extracted from textual documents. The mapping from references to entities is often uncertain.

- Attribute: An attribute is an observed property of an individual reference, for example the recorded name, address or phone number of a person reference in a social network database. Attributes of a reference are mostly derived 
from corresponding attributes of the underlying entities. However, in many applications we do not have attributes which serve as identifiers for entities and this leads to uncertainty in the mapping from references to entities.

- Relationship: When multiple references are observed together, or in the same context, that forms a co-occurrence relationship between those references. For example, we have names of different people occurring in the same email or names of researchers occurring as co-author names in publications. When viewing the data as a graph, we alternatively use the term hyper-edge to refer to a co-occurrence relationship that connects many reference nodes. Co-occurrences usually happen as a result of ties or links between the underlying entities, such as a friendships between people. I sometimes use the term relationship to refer to these ties between entities as well. But, unless explicitly mentioned, relationship will be used to mean a co-occurrence relationship between multiple references.

- Group: A group is a collection of entities that have close ties between themselves. For example, we can have a group of friends or a group of colleagues who inter-act frequently. Entities can belong to multiple groups at the same time. Groups are only observed indirectly through co-occurrences that mostly happen between references to entities that belong to the same group. The observed co-occurrence relations in the data provide evidence for discovering the group structures among the entities, and the group evidence in turn helps in improved resolution of the references. 


\subsection{Specific Contributions and Organization of the Dissertation}

The specific contributions of this dissertation are as follows:

1. In this dissertation, I define the problem of collective entity resolution using relationships between references. I introduce two different approaches for unsupervised collective entity resolution. The relational clustering algorithm combines attributes with relationships in a novel way to measure similarities between clusters. The probabilistic $L D A-E R$ model uses group structures among underlying entities to resolve references. I propose novel inference algorithms for this model using sampling approaches.

2. I perform extensive experiments on multiple real and synthetic datasets and compare against various baselines to demonstrate that collective entity resolution significantly improves performance over traditional approaches that make use of attributes of references. Using synthetically generated data, I also explore structural and other properties of datasets to investigate characteristics that favor or adversely affect collective resolution.

3. I motivate the problem of query-time entity resolution where entities are resolved on the fly for answering queries over unresolved databases. I formally analyze the dependencies arising from collective resolution and show the validity of a limited-depth recursive expansion process for answering queries. I propose adaptive algorithms that identify the most informative related references for resolving queries collectively. This enables query-time resolution 
while preserving the performance benefits of collective resolution.

4. As an application of entity resolution in the domain of computational linguistics, I investigate the problem of word sense disambiguation in natural language documents. Using aligned translation threads from parallel texts, I focus on collective sense resolution in multiple languages. I propose two probabilistic models for word sense disambiguation using translations that outperform existing unsupervised approaches for this problem.

There is a large body of related work on entity resolution, as I discuss in Chapter 6. But this dissertation stands out in more ways than one. Though entity resolution problem has been around for many years, my relational clustering approach [8] is one of the first to make use of relationships for collective or joint resolution. Since then, the use of relationships and even collective solutions for this problem have gained in popularity, and both probabilistic and non-probabilistic approaches have been proposed by other researchers. Therefore, it is important to appreciate the contributions and the novelty of this dissertation in the light of this related research. The probabilistic model that I propose is one of the very few generative models for noisy and uncertain co-occurrence relations, and unlike most other models, my learning algorithm is completely unsupervised. LDA-ER is also unique in that it uses a group variable to model relationships between entities, thereby avoiding expensive pair-wise relationship variables. The other approach based on relational clustering is unique in that it poses collective relational entity resolution as a distance/similarity-based clustering problem. The problem of collective clustering 
has previously received little attention in the literature, and my proposed approach of using similarity-measures that accommodate collective decisions is one of the first solutions to be proposed. Unlike most other work on entity resolution, efficiency is a key concern for all my approaches. This finally culminates in the formulation of the query-time entity resolution problem. Looking beyond entity resolution, clustering at query time in the presence of relationships has not been studied in the literature to the best of my knowledge. Motivating this problem and proposing a working solution for it also counts as a significant contribution of this dissertation.

The rest of the dissertation is organized as follows. The next two chapters, Chapter 2 and Chapter 3, discuss the two approaches to collective entity resolution. In Chapter 2, I first motivate the entity resolution problem using a bibliographic example and formulate the problem. I also discuss different approaches based on attributes and relationships that may be used to address the entity resolution problem before going into the details of the relational clustering algorithm. Next, in Chapter 3, I describe and evaluate the probabilistic approach to collective entity resolution. Then I move on to the problem of entity resolution for queries in Chapter 4, where I first motivate the problem and then discuss, analyze and evaluate algorithms for query-time entity resolution. Chapter 5 discusses the word sense disambiguation problem. I review related work in entity resolution in Chapter 6 and then finally discuss potential future directions and conclude in Chapter 7. 


\section{Chapter 2}

\section{Relational Clustering for Collective Entity Resolution}

In this chapter, I propose the first solution to the collective entity resolution problem, which is based on a novel unsupervised relational clustering algorithm. Before describing the proposed approach, I first present a more realistic motivating example for entity resolution using the relations between references in Section 2.1 and formalize the relational entity resolution problem in Section 2.2. I explore and compare different approaches for entity resolution and formulate collective relational entity resolution as a clustering problem in Section 2.3. I propose novel relational similarity measures for collective relational clustering in Section 2.4. I discuss the clustering algorithm in further detail in Section 2.5. In Section 2.6, I describe experimental results using the different similarity measures on multiple real-world datasets. I also present detailed experiments on synthetically generated data to identify data characteristics that indicate when collective resolution should be favored over the more naive approaches and finally conclude in Section 2.7.

\subsection{Motivating Example for Entity Resolution Using Relationships \\ I consider as our motivating example the problem of resolving the authors in a database of academic publications similar to DBLP, CiteSeer or PubMed. Consider}




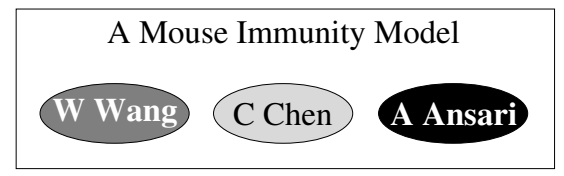

Paper 1

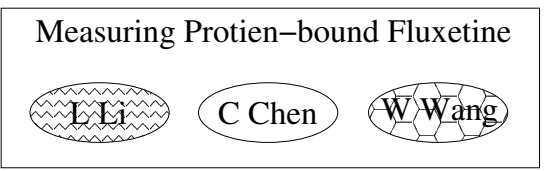

Paper 3

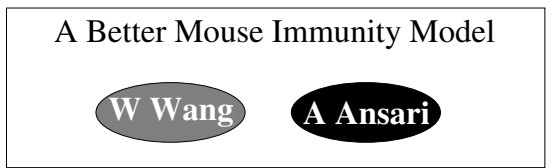

Paper 2

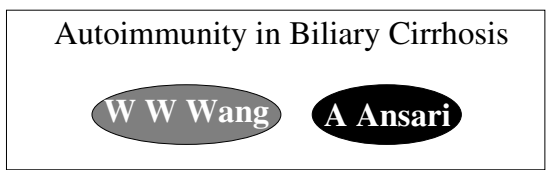

Paper 4

Figure 2.1: The references in different papers in the bibliographic example. References to different entities are shaded differently.

the following set of four papers, which I will use as a running example:

1. W. Wang, C. Chen, A. Ansari, "A mouse immunity model"

2. W. Wang, A. Ansari, "A better mouse immunity model"

3. L. Li, C. Chen, W. Wang, "Measuring protein-bound fluxetine"

4. W. W. Wang, A. Ansari, "Autoimmunity in biliary cirrhosis"

Now imagine that we would like to find out, given these four papers, which of these author names refer to the same author entities. This involves determining whether paper 1 and paper 2 are written by the same author named Wang, or whether they are different authors. We need to make similar decisions about the Wang from paper 3 and the Wang from paper 4, and all pairwise combinations. We need to answer similar questions about the other author names Ansari and Chen as well.

In this example, it turns out there are six underlying author entities, Wang 1 and Wang2, Chen 1 and Chen2, Ansari and Li. The three references with the 
name 'A. Ansari' correspond to author Ansari and the reference with name 'L. Li' to author $L i$. However, the two references with name 'C. Chen' map to two different authors Chen 1 and Chen2. Similarly, the four references with name 'W. Wang' or 'W. W. Wang' map to two different authors. The 'Wang' references from the first, second, and fourth papers correspond to author Wang 1 , while that from the third paper maps to a different author Wang2. This is shown pictorially in Figure 2.1, where references which correspond to the same authors are shaded identically.

There are two different subproblems that are of interest in solving the entity resolution problem. One is figuring out for any author entity the set of different name references which may be used to refer to the author. I refer to this as the identification problem. For example, for a real-world entity with the name 'Wei Wei Wang', her name may come up as 'Wei Wang', 'Wei W. Wang', 'W. W. Wang', 'Wang, W. W.' and so on. There may also be errors in the data entry process, so that the name may be incorrectly recorded as 'W. Wong' or 'We Wang' etc.

In addition to the reconciliation of different looking names which refer to the same underlying entity, a second aspect of entity resolution problem is distinguishing references that have very similar and sometimes exactly the same name and yet refer to different underlying entities. I refer to this as the disambiguation problem. An example of this is determining that the 'W. Wang' of paper 1 is distinct from the 'W. Wang' of paper 3. The extent of the disambiguation problem depends on the domain. The problem can be exacerbated by the use of abbreviations; many databases (for example PubMed) store only abbreviated names. 

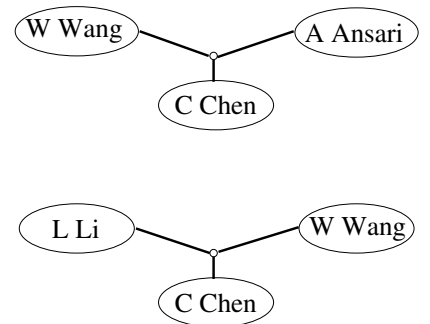

(a)
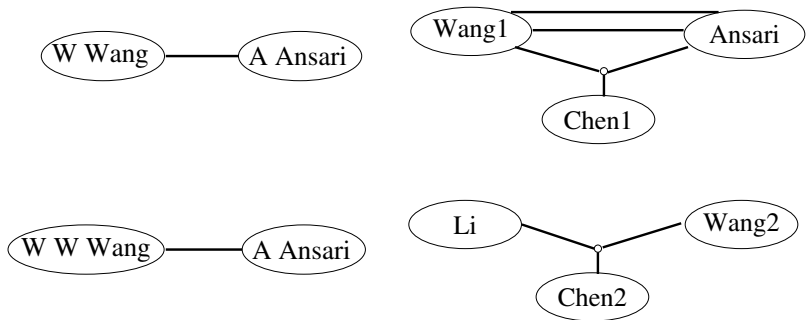

(b)

Figure 2.2: (a) The reference graph and (b) the entity graph for the author resolution example.

The aim is to make use of the relationships that hold among the observed references to resolve them better, and to solve both the identification and disambiguation problem at the same time. As in the case of the census example, we can represent the relationships as a graph where the vertices represent the author references and the hyper-edges represent the co-authorship relations that hold between them in the dataset. Figure 2.2(a) shows the reference graph for the bibliographic example, where the nodes are the references and hyper-edges in the graph indicate which references co-occur. Given this graph representation for the data, the goal is to take the hyper-edges into account to better partition the references into entities. Now, in addition to the similarity of the attributes of the references, I consider their relationships as well. In terms of the graph representation, two references that have similar attributes are more likely to be the same entity if their hyper-edges connect to the same entities as well. To see how this can help, observe in Figure 2.1(a) that the Wang references in papers 1, 2 and 4 collaborate with Ansari's who correspond to the same author. This makes it more likely that they are the same entity. In contrast, the 'Wang' from paper 3 collaborates with different authors, which suggests 
that it does not refer to the same person as the other cases.

But it seems that we are stuck with a 'chicken-and-egg' problem. The identity of a reference depends on those of its collaborators, and the identity of the collaborators depends on the identity of the reference itself. So where do we begin? Intuitively, we start with the resolutions that we are most confident about. For instance, two references with the name 'A. Ansari' are more likely to be the same because 'Ansari' is a common name, in contrast to references with common names such as 'Chen', 'Li' or 'Wang'. This then provides additional evidence for merging other references. In the example after consolidating the 'Ansari's, the 'Wang' references from paper 1, 2 and 4 have a common co-author, which provides provides evidence for consolidating them. The entity resolution algorithm incrementally constructs the entity graph by considering as evidence the entity relationships that it has already discovered in earlier iterations. Figure 2.2(b) shows the resulting entity graph for the example after all the references have been correctly resolved.

\subsection{Entity Resolution Using Relationships: Problem Formulation}

In this section, I describe the notation I use for describing the relational entity resolution problem. In the entity resolution problem, we are given a set of references $\mathcal{R}=\left\{r_{i}\right\}$, where each reference $r$ has attributes $r . A_{1}, r . A_{2}, \ldots, r . A_{k}$. The references correspond to some set of unknown entities $\mathcal{E}=\left\{e_{i}\right\}$. I introduce the notation $r$. $E$ to refer to the entity to which reference $r$ corresponds. The problem is to recover the hidden set of entities $\mathcal{E}=\left\{e_{i}\right\}$ and the entity labels $r$. $E$ for individual references 

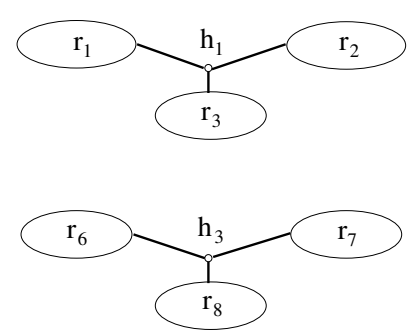

(a)
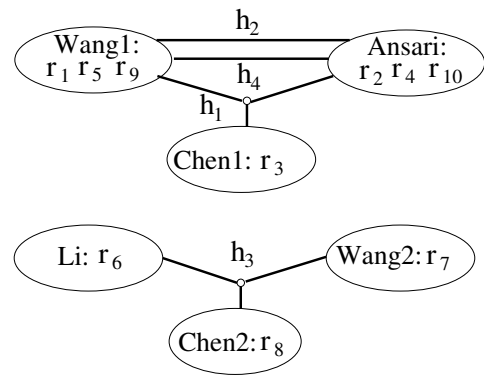

(b)

Figure 2.3: (a) A more abstract representation of the reference graph for the author resolution example; the r's are references and the h's are hyper-edges. (b) An abstract representation for the entity graph for the author resolution example; the nodes are the entities, the set of references they correspond to are listed, and the h's are hyper-edges.

given the observed attributes of the references. In addition to the attributes, I assume that the references are not observed independently, but that they co-occur. I describe the co-occurrence with a set of hyper-edges $\mathcal{H}=\left\{h_{i}\right\}$. Each hyper-edge $h$ may have attributes as well, which I denote $h . A_{1}, h . A_{2}, \ldots, h . A_{l}$, and I use $h . R$ to denote the set of references that it connects. A reference $r$ can belong to zero or more hyper-edges and I use $r . H$ to denote the set of hyper-edges in which $r$ participates. In this paper, I only discuss entity resolution when each reference is associated with zero or one hyper-edge, but in other domains it is possible for multiple hyper-edges to share references. For example, if we have paper, author and venue references, then a paper reference may be connected to multiple author references and also to a venue reference.

Let us now illustrate how the running example is represented in this notation. Figure 2.3(a) shows the references and hyper-edges. Each observed author name corresponds to a reference, so there are ten references $r_{1}$ through $r_{10}$. In this case, 
the names are the only attributes of the references, so for example $r_{1} . A$ is "W. Wang", $r_{2} . A$ is "C. Chen" and $r_{3} . A$ is "A. Ansari". The set of true entities $\mathcal{E}$ is $\{$ Ansari, Wang1,Wang2, Chen 1, Chen $2, L i\}$ as shown in Figure 2.3(b). References $r_{1}, r_{5}$ and $r_{9}$ correspond to $W$ ang 1 , so that $r_{1} . E=r_{5} . E=r_{9} . E=W a n g 1$. Similarly, $r_{2} \cdot E=r_{4} \cdot E=r_{10} \cdot E=$ Ansari and $r_{3} \cdot E=$ Chen 1 and so on. There are also the hyper-edges $\mathcal{H}=\left\{h_{1}, h_{2}, h_{3}, h_{4}\right\}$, one for each paper. The attributes of the hyperedges in this domain are the paper titles; for example, $h_{1} \cdot A_{1}=$ "A Mouse Immunity Model". The references $r_{1}$ through $r_{3}$ are associated with hyper-edge $h_{1}$, since they are the observed author references in the first paper. This is represented as $h_{1} . R=\left\{r_{1}, r_{2}, r_{3}\right\}$. Also, this is the only hyper-edge that each of these references participate in. So $r_{1} \cdot H=r_{2} \cdot H=r_{3} \cdot H=\left\{h_{1}\right\}$. I similarly represent the hyper-edge associations of the other references.

\subsection{Entity Resolution Approaches}

In this section, I compare and contrast existing entity resolution approaches. I distinguish between attribute-based, naive relational and collective relational entity resolution. While the attribute-based approach considers only the attributes of the references to be matched, the naive relational approach considers attribute similarities for related references as well. In contrast, the collective relational approach resolves related references jointly. I consider each approach in detail one by one. 


\subsubsection{Attribute-based Entity Resolution}

This is the traditional approach $[44,30]$ where similarity $\operatorname{sim}_{A}\left(r_{i}, r_{j}\right)$ is computed for each pair of references $r_{i}, r_{j}$ based on their attributes and only those pairs that have similarity above some threshold are considered co-referent. I use the ab-

breviation A to refer to the attribute-based approach. Additionally, transitive closure may be taken over the pair-wise decisions. I denote this approach as $\mathbf{A}^{*}$.

Several sophisticated similarity measures have been developed for names, and popular TF-IDF schemes may be used for other textual attributes like keywords. The measure that works best for each attribute can be used. Finally, a weighted combination of the similarities over the different attributes for each reference can be taken for the combined attribute similarity between two references. In our example, the approach A may allow us to decide that the 'W. Wang' references $\left(r_{1}, r_{5}\right)$ are co-referent. I may also decide using A that 'W. Wang' and 'W.W. Wang' $\left(r_{1}, r_{9}\right)$ are co-referent, but not as confidently. However, as I have already discussed, attributes are often insufficient for entity resolution, particularly for the disambiguation aspect of the problem. In our example, $\mathbf{A}$ is almost certain to mark the two 'W. Wang' references $\left(r_{1}, r_{7}\right)$ as co-referent, which is incorrect.

\subsubsection{Naive Relational Entity Resolution}

The simplest way to use relationships to resolve entities is to treat related references as additional attributes for matching. For instance, to determine if two author references in two different papers are co-referent, we can compare the names 
of their co-authors. In our running example, the naive relational decision about the references 'W. Wang' and 'W. W. Wang', would consider that both have co-authors with the name 'A. Ansari'. I refer to this approach as NR. As before, transitive closure can be taken over the pair-wise decisions for NR. I refer to the transitive closure as $\mathbf{N R}^{*}$.

A similar idea has been used in the context of matching in dimensional hierarchies [3]. I generalize the idea for unordered relationships and define naive relational similarity $\operatorname{sim}_{N R}\left(h_{i}, h_{j}\right)$ between two hyper-edges $h_{i}$ and $h_{j}$ as the best pair-wise attribute match between their references. Since the references in any hyper-edge are not ordered, each reference $r \in h_{i}$ can be matched to any reference $r^{\prime} \in h_{j}$. So for each reference $r \in h_{i}$ I find the best match to $h_{j}$ :

$$
\operatorname{sim}_{A}\left(r, h_{j}\right)=\max _{r^{\prime} \in h_{j}} \operatorname{sim}_{A}\left(r, r^{\prime}\right)
$$

For symmetry, I also compute the best match to hyper-edge $h_{i}$ for each reference in $h_{j}$ and then take the average over all of the references in the two hyper-edges to get $\operatorname{sim}_{N R}\left(h_{i}, h_{j}\right)$. I then use this similarity measure between two hyper-edges to find the naive relational similarity $\operatorname{sim}_{N R}\left(r_{i}, r_{j}\right)$ between two references $r_{i}$ and $r_{j}$ by matching their hyper-edges. When each reference belongs to just one hyper-edge, $\operatorname{sim}_{N R}\left(r_{i}, r_{j}\right)$ can be computed simply as $\operatorname{sim}_{N R}\left(r_{i} . H, r_{j} . H\right)$. Otherwise, I need to make pair-wise comparisons between their hyper-edges. Finally, I take a simple linear combination of the attribute match $\operatorname{sim}_{A}\left(r_{i}, r_{j}\right)$ and the naive relational match $\operatorname{sim}_{N R}\left(r_{i}, r_{j}\right)$ to get combined similarity for two references $r_{i}$ and $r_{j}$ :

$$
\operatorname{sim}\left(r_{i}, r_{j}\right)=(1-\alpha) \times \operatorname{sim}_{A}\left(r_{i}, r_{j}\right)+\alpha \times \operatorname{sim}_{N R}\left(r_{i}, r_{j}\right), \quad 0 \leq \alpha \leq 1
$$


While the naive relational approach improves significantly on the attributebased approach, it can be misled in domains where most names are frequent and hyper-edges are dense. In our example, the two 'W. Wang' references, $r_{1}$ and $r_{7}$ are not co-referent, though they have co-authors with matching names 'C. Chen'. Since I only match the strings, naive relational similarity returns a high match value. This may incorrectly lead to the decision that $r_{1}$ and $r_{7}$ are co-referent.

\subsubsection{Collective Relational Entity Resolution}

The problem with the naive relational approach is that it does not reason about the identities of the related references. For the two 'Wang' references in the earlier example, the two 'C. Chen' co-authors match regardless of whether they refer to Chen1 or Chen2. The correct evidence to use here is that the 'Chen's are not co-referent. In such a setting, in order to resolve the 'W. Wang' references, it is necessary to resolve the ' $\mathrm{C}$ Chen' references as well, and not just consider their name similarity. This is the goal of collective relational entity resolution $\mathbf{C R}$, where resolutions are not made independently, but instead one resolution decision affects other resolutions via hyper-edges. I now motivate entity resolution as a clustering problem and propose a relational clustering algorithm for collective relational entity resolution.

Given any similarity measure between pairs of references, entity resolution can be posed as a clustering problem where the goal is to cluster the references so that only those that correspond to the same entity are assigned to the same cluster. A 
greedy agglomerative clustering algorithm is often used, where at any stage of the process, the current set $\mathcal{C}=\left\{c_{i}\right\}$ of entity clusters reflects the current belief about the mapping of the references to entities. I use $r . C$ to denote the current cluster label for a reference; references that have the same cluster label correspond to the same entity. So far, I have discussed similarity measures for attributes; for a clustering algorithm, I need to define similarities between clusters of references. The goal is to use clustering for collective entity resolution. I now look at how we can define similarity measures between clusters for this purpose.

I define the similarity of two clusters $c_{i}$ and $c_{j}$ as:

$$
\operatorname{sim}\left(c_{i}, c_{j}\right)=(1-\alpha) \times \operatorname{sim}_{A}\left(c_{i}, c_{j}\right)+\alpha \times \operatorname{sim}_{R}\left(c_{i}, c_{j}\right), \quad 0 \leq \alpha \leq 1
$$

where $\operatorname{sim}_{A}()$ is the similarity of the attributes and $\operatorname{sim}_{R}()$ is the relational similarity between the references in the two entity clusters. On analyzing Eq. (2.2), we can see that it reduces to attribute-based similarity for $\alpha=0$. Also, the relational aspect of the similarity measures distinguishes it from the naive relational similarity measure from Eq. (2.1). While naive relational similarity measures the attribute similarity of the related references, here I consider the labels of related clusters that represent entities. This similarity is dynamic in nature, which is one of most important and interesting aspects of the collective approach. In contrast to attribute-based and naive relational resolution, where the similarity between two references is fixed, for collective resolution, the similarity of two references depends on the current cluster labels of the related references and therefore changes as the labels are updated. In our example, the similarity of the two references 'W. Wang' and 'W. W. Wang' 
increase once the Ansari references are given the same cluster label.

As I have mentioned earlier, similarity measures for attributes have been studied in great detail. The focus is on measuring relational similarity between two clusters of references. The references in each cluster $c$ are connected to other references via hyper-edges. For collective entity resolution, relational similarity considers the cluster labels of all these connected references. Recall that each reference $r$ is associated with one or more hyper-edges in $\mathcal{H}$. Therefore, the set of hyper-edges $c . H$ that I need to consider for an entity cluster $c$ is defined as

$$
c . H=\bigcup_{r \in \mathcal{R} \wedge r . C=c}\{h \mid h \in \mathcal{H} \wedge r \in h . R\}
$$

These hyper-edges connect $c$ to other clusters. The relational similarity for two clusters $c_{1}$ and $c_{2}$ needs to compare this connectivity pattern to other clusters for $c_{1}$ and $c_{2}$.

For any cluster $c$, the set of other clusters to which $c$ is connected via its hyper-edge set $c . H$ form the neighborhood $\operatorname{Nbr}(c)$ of cluster $c$ :

$$
N b r(c)=\bigcup_{h \in c . H, r \in h . R}\left\{c_{j} \mid c_{j}=r . C\right\}
$$

This defines the neighborhood as a set of related clusters, but the neighborhood can also be defined as a bag or multi-set, in which the multiplicity of the different neighboring clusters is preserved. I will use $\mathrm{Nbr}_{B}\left(c_{i}\right)$ to denote the bag of neighboring clusters. In our example in Figure 2.3(b), the neighborhood of the cluster for Wang 1 consists of the clusters for Ansari and Chen 1 ; alternatively it is the bag of clusters $\{$ Ansari, Ansari, Ansari, Chen 1$\}$. Note that I do not constrain the defini- 
tion of the neighborhood of a cluster to exclude the cluster itself. In Section 2.4.6, I discuss how such constraints can be handled when required by the domain.

For the relational similarity between two clusters, I look for commonness in their neighborhoods. This can be done in many different ways, as I explore in the following section.

\subsection{Neighborhood Similarity Measures for Collective Resolution}

We have seen how the neighborhood of a cluster of references can be represented as a set (or alternatively as a multi-set) of cluster labels and that we can compute relational similarity between two clusters by considering the similarity of their neighborhoods. Many different metrics have been proposed and evaluated in the literature for measuring commonness between sets; for example Liben-nowell and Kleinberg [68] study their use for prediction tasks in social networks. Here I adapt and modify some of these measures and study their applicability for entity resolution.

\subsubsection{Common Neighbors}

This is the simplest approach for measuring commonness between sets and counts the number of elements that occur in both. For two clusters $c_{i}$ and $c_{j}$, their common neighbor score is defined as

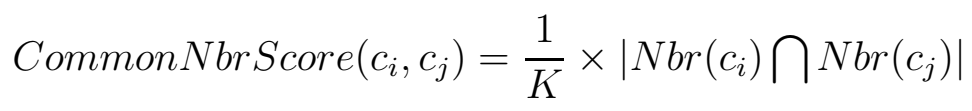


where $K$ is a large enough constant such that the measure is less than 1 for all pairs of clusters. For two references 'John Smith' and 'J. Smith', where attribute similarity is not very informative, this score measures the overlap in their connected entities. The greater the number of common entities, the higher the possibility that the two references refer to the same entity as well.

This definition ignores the frequency of connectivity to a neighbor. Suppose 'John Smith' has collaborated with the entity 'J. Brown' several times, while 'J. Smith' has done so only once. To investigate if this information is relevant for entity resolution, I also define a common neighbor score with frequencies that takes into account multiple occurrences of common clusters in the neighborhoods:

$$
\text { CommonNbrScore }+\operatorname{Fr}\left(c_{i}, c_{j}\right)=\frac{1}{K^{\prime}} \times\left|N b r_{B}\left(c_{i}\right) \bigcap N b r_{B}\left(c_{j}\right)\right|
$$

\subsubsection{Jaccard Coefficient}

The main shortcoming of the common neighbor score is the normalizing constant $K$ which is the same over all pairs of clusters. Consider the situation where we have two 'John Smith' clusters, $c_{1}$ and $c_{2}$, both of which have the same number of

neighbors in common with the 'J. Smith' cluster $c_{3}$. Then they are equally similar to $c_{3}$ in terms of the common neighbor score. Suppose that all of $c_{1}$ 's neighbors are shared with $c_{3}$, while $c_{2}$ has a very large neighborhood and only a small fraction of it is shared with $c_{3}$. When entities have large neighborhoods, finding shared neighbors by chance becomes more likely. In this case, we may want the similarity between $c_{1}$ and $c_{3}$ to be greater than the similarity between $c_{2}$ and $c_{3}$. We can get around this 
issue by taking into account the size of neighborhood. This gives us the Jaccard coefficient for two clusters:

$$
\operatorname{JaccardCoeff}\left(c_{i}, c_{j}\right)=\frac{\left|\operatorname{Nbr}\left(c_{i}\right) \cap N b r\left(c_{j}\right)\right|}{\left|\operatorname{Nbr}\left(c_{i}\right) \cup N b r\left(c_{j}\right)\right|}
$$

As before, we may consider neighbor counts to define the Jaccard coefficient with frequencies, JaccardCoeff $+\operatorname{Fr}\left(c_{i}, c_{j}\right)$, by using $N b r_{B}\left(c_{i}\right)$ and $N b r_{B}\left(c_{j}\right)$ in the definition.

\subsubsection{Adamic/Adar Similarity}

Both the common neighborhood measure and Jaccard coefficient consider all cluster labels in the neighborhood as equally important and significant for determining co-reference. However this is not always desirable. If a cluster is frequently linked with many different clusters, then its presence in a shared neighborhood is not as significant as a cluster which is less frequent. This is similar to the idea behind 'inverse document frequency' in the commonly used TF-IDF scheme in information retrieval. Adamic and Adar [1] use this idea for predicting friendship from web-page features. They proposed a similarity measure between two web-pages $X$ and $Y$ that individually considers the significance of each element that they share and assigns weights to them accordingly. This has come to be called the Adar / Adamic score:

$$
\operatorname{similarity}(X, Y)=\sum_{\text {shared feature } z} \frac{1}{\log (\text { frequency }(z))}
$$

Liben-nowell and Kleinberg [68] adapted this idea for the task of link prediction in social networks considering node neighborhoods, where they used the size of a 
node's neighborhood for measuring frequency or commonness. I generalize this idea to propose a class of Adar/Adamic measures for entity resolution. If the 'uniqueness' of a cluster label $c$ (or a shared feature, in general) is denoted as $u(c)$, then I define the Adar similarity score of two clusters $c_{i}$ and $c_{j}$ as

$$
\operatorname{Adar}\left(c_{i}, c_{j}\right)=\frac{\sum_{c \in N b r\left(c_{i}\right) \cap N b r\left(c_{j}\right)} u(c)}{\sum_{c \in N b r\left(c_{i}\right) \cup N b r\left(c_{j}\right)} u(c)}
$$

where the denominator normalizes the score. Now the Jaccard coefficient can be viewed as a special case of the Adar score when all nodes are equally unique. Also, observe that without the normalization Eq. (2.6) reduces to the similarity score of Liben-nowell and Kleinberg [68] for

$$
u(c)=\frac{1}{\log (|N b r(c)|)}
$$

I refer to Adar score that uses this definition of uniqueness as the AdarNbr score. As before, I evaluate two versions, AdarNbr that considers the set of neighbors and AdarNbr+Fr that takes into account the multiplicity of the neighbors.

\subsubsection{Adar Similarity with Ambiguity Estimate}

While using the neighborhood size of a cluster to measure its uniqueness has been shown to work well in link prediction applications, it may not be appropriate for entity resolution. For entity resolution applications, we do not directly know the neighbors for each entity from the data. The true neighborhood size for any entity cluster is known only after the entity graph has been correctly reconstructed. So using the neighborhood size as a measure of uniqueness at any intermediate 
stage of the resolution algorithm is incorrect, and is an overestimate of the actual neighborhood size.

As an alternative, we can use a definition of uniqueness which incorporates a notion of the ambiguity of the names found in the shared neighborhood. To understand what this means, consider two references with name 'A. Aho'. Since 'Aho' can be considered as an 'uncommon' name, they are very likely to be the same person. In contrast, two other references with a common name such as ' $\mathrm{L}$. Li' are less likely to be the same person. So I define the ambiguity $A m b(r . N a m e)$ of a reference name as the probability that multiple entities share that particular name.

Intuitively, clusters which share neighbors with uncommon names are more likely to refer to the same entity and should be considered more similar. I define the uniqueness of a cluster $c$ as inversely proportional to the average ambiguity of its references:

$$
u(c)=\frac{1}{A v g_{r \in c}(A m b(r . N a m e))}
$$

In general, this approach is not specific to names and can be used with any attribute of the references. I refer to an Adar similarity score which uses this definition of uniqueness as AdarName when applied to the set of neighbors and AdarName+Fr to refer to the measure applied to the bag of neighbors.

The Adar-Name measure is defined in terms of the ambiguity of a reference's name. There are a number of ways to estimate the ambiguity of a name. One scheme that works quite well in our domains is to estimate the probability that two randomly picked references with Name $=n$ correspond to different entities. For a 
reference attribute $A_{1}$, denoted $R . A_{1}$, a naive estimate for the ambiguity of a value of $n$ for the attribute is:

$$
A m b\left(r . A_{1}\right)=\frac{\left|\sigma_{R . A_{1}=r . A_{1}}(R)\right|}{|R|},
$$

where $\left|\sigma_{R . A_{1}=r . A_{1}}(R)\right|$ denotes the number of references with value $r . A_{1}$ for $A_{1}$. This estimate is clearly not good since the number of references with a certain attribute value does not always match the number of different entity labels for that attribute. We can do much better if we have an additional attribute $A_{2}$. Given $A_{2}$, the ambiguity for value of $A_{1}$ can be estimated as

$$
A m b\left(r . A_{1} \mid r . A_{2}\right)=\frac{\left|\delta\left(\pi_{R . A_{2}}\left(\sigma_{R \cdot A_{1}=r \cdot A_{1}}(R)\right)\right)\right|}{|R|}
$$

where $\left|\delta\left(\pi_{R . A_{2}}\left(\sigma_{R . A_{1}=r . A_{1}}(R)\right)\right)\right|$ is the number of distinct values observed for $A_{2}$ in references with $R \cdot A_{1}=r \cdot A_{1}$. For example, we can estimate the ambiguity of a last name by counting the number of different first names observed for it. This provides a better estimate of the ambiguity of any value of an attribute $A_{1}$, when $A_{2}$ is not correlated with $A_{1}$. When multiple such uncorrelated attributes $A_{i}$ are available for references, this approach can be generalized to obtain better ambiguity estimates.

\subsubsection{Higher-Order Neighborhoods}

Analysis of the commonness of neighborhoods can be viewed as an investigation of paths of length two between two clusters. I also investigate whether higher-order neighborhoods play a role in detecting co-reference. In addition to the neighborhood similarity measures described, I also evaluate measures which take 
into account collaboration paths of length three. As the clusters change, it becomes computationally infeasible to recompute all paths between all cluster pairs. Instead, I calculate the second order neighborhood $N b r^{2}(c)$ for a cluster $c$ by recursively taking the set union (alternatively, multi-set union) of the neighborhoods of all neighboring clusters: $N b r^{2}(c)=\bigcup_{c^{\prime} \in N b r(c)} N b r\left(c^{\prime}\right)$. For paths of length three to be present between two clusters $c_{i}$ and $c_{j}$, there must be intersections between the $\operatorname{Nbr}\left(c_{i}\right)$ and $\mathrm{Nbr}^{2}\left(c_{j}\right)$, or vice versa. Then, to find the similarity over paths of length 3 or less for $c_{i}$ and $c_{j}$, I take the average of the similarities over length-2 paths and length-3 paths:

$$
\begin{aligned}
\operatorname{Path} 3 \operatorname{Sim}\left(c_{i}, c_{j}\right)= & \frac{1}{3}\left[\operatorname{Jaccard}\left(\operatorname{Nbr}\left(c_{i}\right), \operatorname{Nbr}\left(c_{j}\right)\right)+\operatorname{Jaccard}\left(\operatorname{Nbr}^{2}\left(c_{i}\right), \operatorname{Nbr}\left(c_{j}\right)\right)\right. \\
& \left.+\operatorname{Jaccard}\left(\operatorname{Nbr}\left(c_{i}\right), N b r^{2}\left(c_{j}\right)\right)\right]
\end{aligned}
$$

\subsubsection{Negative Constraints From Relationships}

The common relational structure I have considered so far can be seen as positive evidence for inferring that two author references refer to the same underlying author entity. Additionally, there may be negative constraints as well for entity resolution arising from relationships. For example, in many relational domains, two references appearing in the same hyper-edge cannot refer to the same entity. As a real bibliographic example, consider a paper with co-authors 'M. Faloutsos', 'P. Faloutsos' and 'C. Faloutsos'. Despite the similarity of the uncommon last name, in reality these references correspond to distinct author entities. So, for bibliographic domains, we can add a constraint that two references which co-occur cannot refer 
to the same entity. In domains other than citation data, there may be different relational constraints. In general, we can have a set of negative relational constraints that clusters need to satisfy. I take these into account by setting the similarity between two cluster pairs in Eq. (2.2) to zero if merging them violates any of the relational constraints.

\subsection{Relational Clustering Algorithm}

Given the similarity measure for a pair of reference clusters, I use a greedy agglomerative clustering algorithm that finds the closest cluster pair at each step and merges them. High level pseudo-code for the algorithm is provided in Figure 2.4. In this section, I discuss several important implementation and performance issues regarding relational clustering algorithms for entity resolution.

\subsubsection{Blocking to Find Potential Resolution Candidates}

Unless the datasets are small, it is impractical to consider all possible pairs as potential candidates for merging. Apart from the scaling issue, most pairs checked by an $O\left(n^{2}\right)$ approach will be rejected since usually only about $1 \%$ of all pairs are true matches. Blocking techniques $[52,79,72]$ are usually employed to rule out pairs which are certain to be non-matches. The goal is to separate references into possibly overlapping buckets and only pairs of references within each bucket are considered as potential matches. The relational clustering algorithm uses the blocking method as a black-box and any method that can quickly identify potential matches minimizing 
1.

2.

3.

4.

5.

6.

7.

8.

9.

10.

11.

12.

13.

14.
Find similar references using blocking

Initialize clusters using bootstrapping

For clusters $c_{i}, c_{j}$ such that $\operatorname{similar}\left(c_{i}, c_{j}\right)$

Insert $\left\langle\operatorname{sim}\left(c_{i}, c_{j}\right), c_{j}, c_{j}\right\rangle$ into priority queue

While priority queue not empty

Extract $\left\langle\operatorname{sim}\left(c_{i}, c_{j}\right), c_{i}, c_{j}\right\rangle$ from queue

If $\operatorname{sim}\left(c_{i}, c_{j}\right)$ less than threshold, then stop

Merge $c_{i}$ and $c_{j}$ to new cluster $c_{i j}$

Remove entries for $c_{i}$ and $c_{j}$ from queue

For each cluster $c_{k}$ such that $\operatorname{similar}\left(c_{i j}, c_{k}\right)$

Insert $\left\langle\operatorname{sim}\left(c_{i j}, c_{k}\right), c_{i j}, c_{k}\right\rangle$ into queue

For each cluster $c_{n}$ neighbor of $c_{i j}$

For $c_{k}$ such that $\operatorname{similar}\left(c_{k}, c_{n}\right)$

Update $\operatorname{sim}\left(c_{k}, c_{n}\right)$ in queue

Figure 2.4: High-level description of the relational clustering algorithm 
false negatives can be used. I use a variant of an algorithm proposed by McCallum et al. [72] that I briefly describe below.

The algorithm makes a single pass over the list of references and assigns them to buckets using an attribute similarity measure. Each bucket has a representative reference that is the most similar to all references currently in the bucket. For assigning any reference, it is compared to the representative for each bucket. It is assigned to all buckets for which the similarity is above a threshold. If no similar bucket is found, a new bucket is created for this reference. A naive implementation yields a $O(n(b+f))$ algorithm for $n$ references and $b$ buckets and when a reference is assigned to at most $f$ buckets. This can be improved by maintaining an inverted index over buckets. For example, when dealing with names, for each character I maintain the list of buckets storing last names starting with that character. Then the buckets can be looked up in constant time for each reference leading to an $O(n f)$ algorithm.

\subsubsection{Relational Bootstrapping}

Each iteration of the relational clustering algorithm makes use of clustering decisions made in previous iterations. This is achieved by measuring the shared neighborhood for similar clusters, as explained in Subsection 2.3.3. But if we begin with each reference in a distinct cluster, then initially there are no shared neighbors for references that belong to different hyper-edges. So the initial iterations of the algorithm have no relational evidence to depend on. As a result, the relational 
component of the similarity between clusters would be zero and merges would occur based on attribute similarity alone. Many of such initial merges can be inaccurate, particularly for the references with ambiguous attribute values. To get around this, we need to bootstrap the clustering algorithm such that each reference is not assigned to a distinct cluster. Specifically, if we are confident that some reference pair is coreferent, then they should be assigned to the same initial cluster. However, precision is crucial for the bootstrap process, since the algorithm cannot undo any of these initial merge operations. Observe that this bootstrapping is not necessary for approaches that are not collective. For such approaches, the decision for any reference pair is the same irrespective of the decisions for other pairs. So bootstrapping does not have any effect on subsequent decisions. In this subsection, I describe the bootstrapping scheme for relational clustering that makes use of the hyper-edges for improved bootstrap performance. The basic idea is very similar to the naive relational approach described in Subsection 2.3.2, with the difference that I use exact matches instead of similarity for attributes. To determine if any two references should be assigned to the same initial cluster, I check if their attributes match exactly. For references with ambiguous attributes, I also check if the attributes of their related references match. I now discuss this in greater detail.

The bootstrap scheme goes over each reference pair that is potentially coreferent (as determined by blocking) and determines if it is a bootstrap candidate. First, consider the simple bootstrap scheme that looks only at the attributes of two references. It determines which attribute values are ambiguous and which are not using a data-based ambiguity estimate, as described in Subsection 2.4.4. References 
with ambiguous attribute values are assigned to distinct clusters. Any reference pair whose attribute values match and are not ambiguous is considered to be a bootstrap candidate.

The problem with this simple approach is that it assigns all references with ambiguous attributes to distinct clusters leading to poor recall in datasets with high ambiguity. When hyper-edges are available, they can be used as evidence for bootstrapping of ambiguous references. A pair of ambiguous references form a bootstrap candidate if their hyper-edges match. Two hyper-edges $h_{1}$ and $h_{2}$ are said to have a $k$-exact-match if there are at least $k$ pairs of references $\left(r_{i}, r_{j}\right), r_{i} \in h_{1}$. $R$, $r_{j} \in h_{2} . R$ with exactly matching attributes, i.e. $r_{i} . A=r_{j} . A$. Two references $r_{1}$ and $r_{2}$ are bootstrap candidates if any pair of their hyper-edges have a $k$-exact-match. As a bibliographic example, two references with name 'W. Wang' will not be merged during bootstrapping on the basis of the name alone. However, if the first Wang reference has co-authors 'A. Ansari' and 'C. Chen', and the second Johnson has coauthor 'A. Ansari', then they have a 1-exact-match and, depending on a threshold for $k$, they would be merged. The value of $k$ for the hyper-edge test depends on the ambiguity of the domain. A higher value of $k$ should be used for domains with high ambiguity. Also, when matching hyper-edges, references with ambiguous attributes are not considered for matches in high ambiguity domains. For example, 'C. Chen' may not be considered for a co-author match, since it is a common name.

Other attributes of the references, and also of the hyper-edges, when available, can be used to further constrain bootstrap candidates. Two references are considered only if these other attributes do not conflict. In the bibliographic domain, author 
references from two different papers can be merged only if their languages and correspondence addresses match.

After the bootstrap candidates are identified, the initial clusters are created using the union-find approach so that any two references that are bootstrap candidates are assigned to the same initial cluster. In addition to improving accuracy of the relational clustering algorithm, bootstrapping reduces execution time by significantly lowering the initial number of clusters without finding the most similar cluster-pairs or performing expensive similarity computations.

\subsubsection{Merging Clusters and Updating Similarities}

Once the similar clusters have been identified and bootstrapping has been performed, the algorithm iteratively merges the most similar cluster pair and updates similarities until the similarity drops below some specified threshold. This is shown in lines 5-14 of Figure 2.4. The similarity update steps for related clusters in lines 12-14 are the key steps that distinguish collective relational clustering from a tra-

ditional agglomerative clustering algorithm. In order to perform the update steps efficiently, indexes need to maintained for each cluster. In this section, I describe the data structure that I maintain for this purpose.

In addition to its list of references, I maintain three additional lists with each cluster. First, I maintain the list of similar clusters for each cluster. The second list keeps track of all neighboring clusters. Finally, I keep track of all the queue entries that involve this cluster. For a cluster that has a single reference $r$, the similar 
clusters are those that contain references in the same bucket as $r$ after blocking. Also, the neighbors for this cluster are the clusters containing references that share a hyper-edge with $r$. Then, as two clusters merge to form a new cluster, all of these lists can be constructed locally for the new cluster from those of its parents. All of the update operations from lines 9-14 can be performed efficiently using these lists. For example, updates for related clusters are done by first accessing the neighbor list and then traversing the similar list for each of them.

\subsubsection{Complexity Analysis}

Now that I have described each component of the relational clustering algorithm, let us analyze its time complexity. First, I look at how the number of similarity computations required in lines $3-4$ of Figure 2.4 is reduced by the blocking method. I consider the worst case scenario where the bootstrapping approach does not reduce the number of clusters at all. We need to compare every pair of references within each bucket. Suppose we have $n$ references that are assigned to $b$ buckets with each reference being assigned to at most $f$ buckets. Then using an optimistic estimate, we have $n f / b$ references in each bucket, leading to $O\left((n f / b)^{2}\right)$ comparisons per bucket and a total of $O\left(n^{2} f^{2} / b\right)$ comparisons. In all of this discussion, I assume that the number of buckets is proportional to the number of references, i.e., $b$ is $O(n)$. Additionally, assuming that $f$ is a small constant independent of $n$, we have $O(n)$ computations.

Now, let us look at the time taken by each iteration of the algorithm. To 
analyze how many update/insert operations are required, I assume that for each bucket that is affected by a merge operation, all the $O\left((n f / b)^{2}\right)$ computations need to be redone. Then we need to find out how many buckets may be affected by a merge operation. I say that two buckets are connected if any hyper-edge connects two references in the two buckets. Then if any bucket is connected to $k$ other buckets, each merge operation leads to $O\left(k(n f / b)^{2}\right)$ update/insert operations. This is still only $O(k)$ operations when $f$ is a constant independent of $n$ and $b$ is $O(n)$. Using a binary-heap implementation for the priority queue, the extract-max and each insert and update operation take $O(\log q)$ time, where $q$ is the number of entries in the queue. So the total cost of each iteration of the algorithm is $O(k \log q)$.

Next, I count the total number of iterations that the algorithm may require. In the worst case, the algorithm may have to exhaust the priority queue before the similarity falls below the threshold. So we need to consider the number of merge operations that are required to exhaust a queue that has $q$ entries. If the merge tree is perfectly balanced, then the size of each cluster is doubled by each merge operation and as few as $O(\log q)$ merges are required. However, in the worst case, the merge tree may be $q$ deep requiring as many as $O(q)$ merges. With each merge operation requiring $O(k \log q)$ time, the total cost of the iterative process is $O(q k \log q)$.

Finally, in order to put a bound on the initial size $q$ of the priority queue, I again consider the worst case scenario where bootstrapping does not reduce the number of initial clusters. This resulting in $O\left(n^{2} f^{2} / b\right)$ entries in the queue as shown earlier. Since this is again $O(n)$, the total cost of the algorithm can be bounded by $O(n k \log n)$. The one cost that I have not considered so far is that of bootstrapping. 
We can analyze the bootstrapping by considering it as a sequence of cluster merge operations that do not require any updates or inserts to the priority queue. Then the worst case analysis of the number of iterations accounts for the bootstrapping as well.

To see how this compares against the attribute and naive relational baselines, observe that they need to take a decision for each pair of references in a bucket. This leads to a worst case analysis of $O(n)$ using the same assumptions as before. However, each similarity computation is more expensive for the naive relational approach since it requires a pair-wise match to be computed between two hyperedges.

\subsection{Experimental Evaluation}

I evaluated the proposed relational entity resolution algorithm on several realworld and synthetic datasets. I begin with a description of the experiments on real bibliographic datasets.

\subsubsection{Evaluation on Bibliographic Data}

The real-world datasets describe publications in several different scientific research areas. As in our running example, the goal is to use co-author relationships in the papers to help discover the underlying author entities in the domain and map the author references to the discovered author entities. I first describe the datasets in more detail, and then describe the evaluation and results. 


\subsubsection{Datasets}

CiteSeer: The CiteSeer dataset contains 1,504 machine learning documents with 2,892 author references to 1,165 author entities. For this dataset, the only attribute information available is author name. The full last name is always given, and in some cases the author's full first name and middle name are given and other times only the initials are given. The dataset was originally created by Giles et al. [49] and the version which I use includes the author entity ground truth provided by Aron Culotta and Andrew McCallum, University of Massachusetts, Amherst.

arXiv: The arXiv dataset describes high energy physics publications. It was originally used in KDD Cup 2003 ${ }^{1}$. It contains 29,555 papers with 58,515 references to 9,200 authors. The attribute information available for this dataset is also just the author name, with the same variations in form as described above. The author entity ground truth for this data set was provided by David Jensen, University of Massachusetts, Amherst.

BioBase: The third dataset, describing biology publications, is the Elsevier BioBase dataset $^{2}$ which was used in a recent IBM KDD-Challenge competition. It was created by selecting all Elsevier publications on 'Immunology and Infectious Diseases' between years 1998 and 2001. It contains 156,156 publications with 831,991 author references. Unlike arXiv and CiteSeer that have complete as well as initialed author names, in BioBase, all of the first names and middle names are abbreviated. How-

\footnotetext{
${ }^{1}$ http://www.cs.cornell.edu/projects/kddcup/index.html

${ }^{2}$ http://help.sciencedirect.com/robo/projects/sdhelp/about_biobase.htm
} 
ever the BioBase dataset has other attributes which I use for resolution including: keywords, topic classification, language, country of correspondence and affiliation of the corresponding author. There is a wide variety in the data with 20 languages, 136 countries, 1,282 topic classifications and 7,798 keywords. Entity labels are available only for the top 100 author names with the highest number of references. I evaluate entity resolution performance for BioBase over 10,595 references that have these 100 names, although the collective resolution algorithm requires resolving many of the other references as well.

Ground truth was determined for all of these datasets by the owners using a combination of automatic and manual strategies. The process is not completely free from errors and I had to perform additional cleaning for some CiteSeer and arXiv references in the course of the experiments. For BioBase, $97 \%$ of the labels are estimated to be correct.

Despite the common underlying domain, these datasets vary in a number of important ways. The most important difference is in the inherent uncertainty in the name references. I introduce two measures, which I refer to as ambiguity (corresponding to the disambiguation aspect of resolution) and dispersion (corresponding to the identification aspect), to measure the uncertainty in the data. I consider a name (last name and first initial) to be ambiguous if multiple entities share that name. In CiteSeer dataset, only 3 out of 1185 names are ambiguous and the average number of entities per ambiguous name is 2.33 (the maximum is 3). For arXiv, 374 of the 8737 names are ambiguous, and the average number of entities for these ambiguous names is 2.41 (the maximum is 11). For BioBase, the ambiguity is much 
higher - 84 of the 100 names are ambiguous. The number of entities for each name ranges from 1 to 100 with an average of 32. I introduce dispersion as another measure of the inherent difficulty of the entity resolution problem for a domain. The dispersion for an entity is the number of distinct observed names for each entity. For CiteSeer, 202 out of the 1164 entities have multiple recorded names, the average and maximum dispersion are 1.24 and 8 respectively. In contrast, 3083 out of 8967 entities for arXiv are dispersed over multiple names, and the average dispersion is 1.44 and the maximum is 10 . Since I do not have complete ground truth for the BioBase dataset, dispersion cannot be directly measured. Apart from the level of uncertainty, BioBase differs significantly from the other two datasets in terms of its hyper-edge structure. For BioBase, the number of author references per publication ranges from 1 to 100 with the average being 5.3. In comparison, the averages are 1.92 and 1.98 respectively for CiteSeer and arXiv, the range being 1 to 10 for both.

\subsubsection{Evaluation}

I compare attribute-based entity resolution (A), naive relational entity resolution (NR) that uses attributes of related references, and the proposed collective relational entity resolution (CR). For the first two algorithms, I also consider variants which perform transitive closures over the pair-wise decisions ( $\mathbf{A}^{*}$ and $\left.\mathbf{N R}^{*}\right)$.

In order to measure the performance of the algorithms I consider the correctness of the pair-wise co-reference decisions over all references. I evaluate the pair-wise decisions using the F1 measure, which is the harmonic mean of precision 
and recall. For a fair comparison, I consider the best F1 for each of these algorithms over all possible thresholds for determining matches.

\subsubsection{Experimental Details}

For comparing attributes, which is required for all of the algorithms, I use the Soft TF-IDF similarity for names [30, 16] since it has been shown to perform well for name-based entity resolution. Essentially, Soft TF-IDF augments the TF-IDF similarity for matching token sets with approximate token matching using a secondary string similarity measure. Jaro-Winkler is reported to be the best secondary similarity measure for Soft TF-IDF, but for completeness, I also experiment with the Jaro and the Scaled Levenstein measures. Scaled Levenstein belongs to the editdistance family of similarity measures that assigns unit cost to each edit operation and normalizes the result. Jaro and Jaro-Winkler do not belong to the edit-distance family. The measure the number and order of common characters between strings. Jaro-Winkler is a variant of Jaro that also considers the longest common prefix [30]. They are both well suited for short strings such as personal names. In the case of BioBase, where I had other multi-valued attributes to make use of besides names, I used TF-IDF similarity.

Since for CiteSeer and arXiv it is infeasible to consider all pairs as potential duplicates, blocking is employed to extract the potential matches. This approach retains $\sim 99 \%$ of the true duplicates for both CiteSeer and arXiv. I use bootstrapping for the relational clustering algorithm (CR) for all three datasets. I use bootstrap 
Table 2.1: Performance of different algorithms on the CiteSeer, arXiv and BioBase datasets. I report the mean and the standard deviations (within parenthesis) of the F1 scores obtained using Scaled Levenstein, Jaro and Jaro-Winkler as secondary similarity measure within Soft TF-IDF.

\begin{tabular}{|l|l|l|l|}
\hline & CiteSeer & arXiv & BioBase \\
\hline \hline A & $0.980(0.001)$ & $0.974(0.002)$ & $0.568(0)$ \\
A* $^{*}$ & $0.990(0.001)$ & $0.967(0.003)$ & $0.559(0)$ \\
NR & $0.981(0.006)$ & $0.975(0.016)$ & $0.710(0)$ \\
NR* & $0.991(0.002)$ & $0.972(0.039)$ & $0.753(0)$ \\
Bootstrap H-Amb & $0.217(0)$ & $0.119(0)$ & $0.452(0)$ \\
Bootstrap L-Amb & $0.942(0)$ & $0.977(0)$ & $0.317(0)$ \\
CR & $0.995(0)$ & $0.985(0)$ & $0.819(0)$ \\
\hline
\end{tabular}

for low ambiguity domains with $k=1$ for CiteSeer and arXiv and bootstrap for high ambiguity domains with $k=2$ for BioBase. Recall that the relational clustering algorithm (CR) and the naive relational approach (NR and $\mathbf{N R}^{*}$ ) both use a combination weight $\alpha$. I measure performance of both algorithms at 20 different values of $\alpha$ between 0 and 1 and report the best performance for each of them over this range. For estimating ambiguity of references for AdarName, I use last names with first initial as the secondary attribute. This resulted in very good estimates of ambiguity — the ambiguity estimate for a name is strongly correlated (correlation coeff. 0.8) with the number of entities for that name.

\subsubsection{Results}

Table 2.1 gives an overview of the F1 results of the various algorithms on the three datasets. Recall that the collective relational clustering uses bootstrapping to initialize the clusters. In addition to the three entity resolution approaches that I 
have discussed, I also include for comparison the two boot strapping approaches, one for low ambiguity domains (Bootstrap L-Amb) that is used by CR for CiteSeer and arXiv, and the other for high ambiguity data (Bootstrap H-Amb) that is employed for BioBase. For CR, Table 2.1 records the performance for the best neighborhood similarity measure, which is Jaccard for CiteSeer and arXiv, and AdarName for BioBase. As mentioned earlier, there are several possible choices for the secondary string metric used with the Soft TD-IDF similarity for comparing the names. The results above are the averages using three choices - Scaled Levenstein, Jaro and Jaro-Winkler, with the standard deviation shown in parenthesis.

First, note that the standard deviation in Table 2.1 measures sensitivity of entity resolution performance in terms of the similarity measure used for names. We can see that the results are not very sensitive to the secondary string metric choice. In fact, for collective relational entity resolution $(\mathbf{C R})$, the choice is irrelevant and for the BioBase dataset, in which I have additional features besides the names, the choice is also irrelevant. For the cases in which there were some small differences, Scaled Levenstein was most often, but not always, the best.

Second, looking at the first line in Table 2.1, note the differences in performance for attribute-based entity resolution (A) for the three datasets. The attribute-based algorithm performs remarkably well for the CiteSeer database, and its performance on the arXiv dataset is also respectable. This is in keeping with our earlier observation about the 'hardness' of the datasets in terms of ambiguity and dispersion. The CiteSeer dataset has very little ambiguity and arXiv is only moderately more ambiguous. When datasets are not ambiguous, all dispersed en- 
tities can be successfully identified simply by raising the discrimination threshold for determining duplicates. This increases recall without generating false positives. However, this is not possible when there is significant ambiguity in the data, as we see in the case of BioBase. The performance of the bootstrapping algorithms highlight the same trend. For CiteSeer and arXiv, the low ambiguity version (Bootstrap L-Amb) performs almost as well as the attribute baseline. In a higher ambiguity dataset such as BioBase, it performs many incorrect matches. The high ambiguity bootstrap strategy (Bootstrap H-Amb), which is more cautious for ambiguous references, performs poorly for CiteSeer and arXiv due to low recall but improves performance over Bootstrap L-Amb for BioBase by increasing precision.

Next, observe that the naive relational entity resolution algorithm (NR) which uses attributes of related references in its similarity calculations improves performance over A only marginally for CiteSeer and arXiv, while the improvement is quite significant in the case of BioBase. This suggests that while the attributes of related entries can help in disambiguation in domains with high ambiguity, there may not be much improvement for less ambiguous domains.

The table also shows that the effect of transitive closure on entity resolution performance varies over the datasets. While it improves performance for both $\mathbf{A}$ and NR for CiteSeer and arXiv, in the case of BioBase, it helps NR but not $\mathbf{A}$. A possible explanation is that transitive closure helps in domains with low ambiguity, but it may result in false identifications in higher ambiguity domains.

Finally, note that across all three datasets, the collective relational entity resolution algorithm (CR) performs the best. The gains for the less ambiguous 


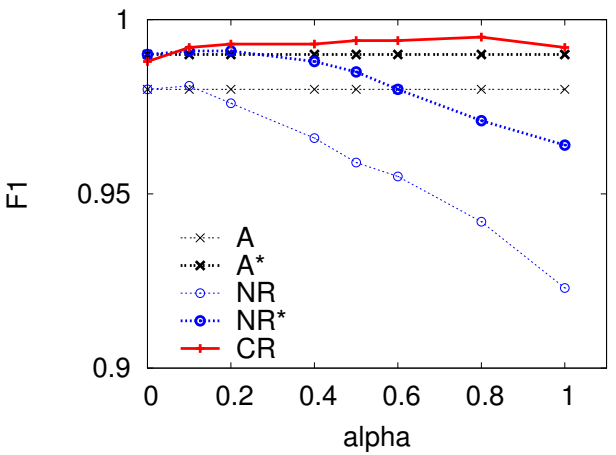

(a)

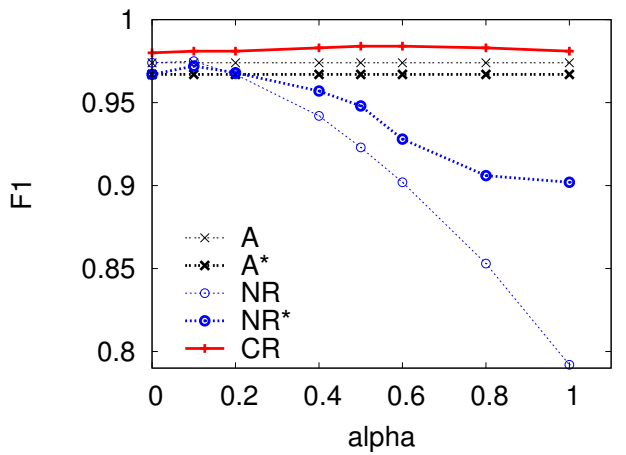

(b)

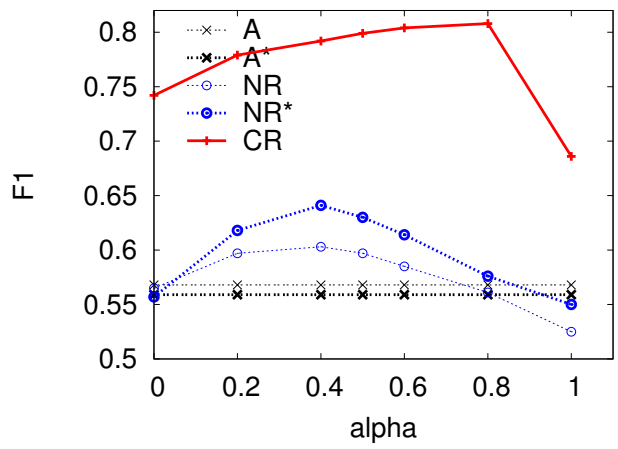

(c)

Figure 2.5: Entity resolution performance at different values of $\alpha$ for (a) CiteSeer, (b) arXiv and (c) BioBase

domains are more modest, while in the most ambiguous domain, the gain is quite significant. In addition, the performance improvements of $\mathbf{C R}$ over $\mathbf{N R}$ highlights the importance over considering the identities of related references rather than just their attributes. Also, since the performance is insensitive to the choice of attribute similarity used, overall $\mathbf{C R}$ is more robust than $\mathbf{A}$ and $\mathbf{N R}$.

Recall that $\mathbf{C R}, \mathbf{N R}$ and $\mathbf{N R}^{*}$ involve a weighting parameter $\alpha$ for combining attribute and relational similarity. As mentioned earlier, the numbers in Table 2.1 
record the best performance over different values of $\alpha$ for each of these algorithms. The best performance is not always achieved for the same value of $\alpha$ for different datasets or for the 100 different reference names in BioBase. In Figure 2.5 we see how the performance of the different algorithms changes over different values of $\alpha$ for the three datasets. For BioBase, I plot the average performance over all 100 reference names for a particular value of $\alpha$. As a reference, I also show the performances of $\mathbf{A}$ and $\mathbf{A}^{*}$ which do not depend on $\alpha$. We can make two interesting observations from the plots. First, the relational clustering algorithm $\mathbf{C R}$ consistently outperforms the naive relational baselines $(\mathbf{N R})$ and $\left(\mathbf{N R}^{*}\right)$ for all values of $\alpha$ for all three datasets. Secondly, for CiteSeer and arXiv, the naive relational approach outperforms the attribute-only baseline only marginally for small values of $\alpha$ and then its performance drops significantly at higher values. It is more stable for BioBase but performs still drops below the attribute-only baseline for high values of $\alpha$. The performance of CR is significantly more stable over varying $\alpha$ for all three datasets. This is another validation of the usefulness of resolving related references instead of considering their similarities.

Now I explore $\mathbf{C R}$ in more depth, by comparing the performance of the algorithm using different graph-based similarity measures. Table 2.2 shows the performance of the collective relational entity resolution with the different proposed measures on the three datasets. There is little difference in performance on the CiteSeer and arXiv datasets. The simplest measure, Common, correctly retrieves almost all duplicates in CiteSeer. Recall that due to the 'blocking' approach, 100\% recall - and therefore an F1 score of 1.0 - is not attainable for these two datasets. 
Table 2.2: Performance (F1) for collective relational entity resolution using different neighborhood similarity measures in the three bibliographic datasets.

\begin{tabular}{|l|l|l|l|}
\hline & CiteSeer & arXiv & BioBase \\
\hline \hline Common & 0.994 & 0.984 & 0.814 \\
Common+Fr & 0.994 & 0.984 & 0.816 \\
Jaccard & 0.994 & 0.985 & 0.818 \\
Jaccard+Fr & 0.995 & 0.985 & 0.818 \\
AdarNbr & 0.994 & 0.984 & 0.815 \\
AdarNbr+Fr & 0.994 & 0.984 & 0.816 \\
AdarName & 0.994 & 0.985 & 0.819 \\
AdarName+Fr & 0.995 & 0.984 & 0.817 \\
Path3Sim & 0.994 & 0.984 & 0.812 \\
\hline
\end{tabular}

There is a bit more of an impact on the BioBase results. The numbers do not provide enough evidence to validate the use of frequencies $(+\mathbf{F r})$ for comparing neighborhoods. It improves performance in some cases and affects it adversely in others. So in the following discussion, I concentrate on the basic similarity measures where the cluster neighborhood is treated as a set, rather than as a multi-set. We can make four observations:

- Jaccard similarity improves performance over Common neighbors. Recall that the difference between the two is in the normalization. This shows the importance of considering the size of the common neighborhood as a fraction of the entire neighborhood.

- AdarNbr performs worse than Jaccard. Recall that Adar similarity considers the importance or uniqueness of each cluster in the shared neighborhood. I pointed out that the 'connectedness' of a shared neighbor is not a reliable indicator in our case, since the graph is consolidated over iterations and new 


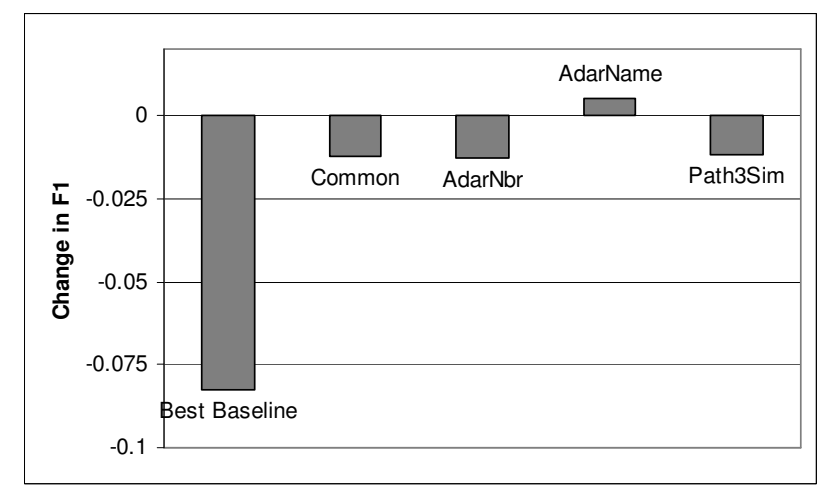

Figure 2.6: Comparison of different relational similarity measures against Jaccard over only the affected instances in BioBase in each case

hyper-edges are added to each cluster. This is validated by the drop in performance as we move to AdarNbr from Jaccard.

- AdarName performs the best over all the graph-based similarity measures. Recall that AdarName attempts to capture the 'uniqueness' of a cluster of references, and this, combined with Adar similarity, works the best of all the neighborhood similarity measures on BioBase.

- Path3Sim has the lowest performance of all the graph-based measures. Recall that Path3Sim explores second order neighborhoods for detecting co-reference. This suggests that in dense collaboration graphs with many ambiguous entities, where distinct entities with similar attributes have common higher order neighbors, going beyond immediate neighborhood can hurt entity resolution performance.

The numbers in Table 2.2 show the average performance of the different measures over all 100 instances in BioBase. However, it is not the case that performance 
is affected for every instance by changing the similarity measure. For example, performance changes in only 22 of the 100 instances when using Jaccard similarity instead of AdarName similarity, as compared to 80 for Jaccard compared to the baseline attribute-based similarity measure. In Figure 2.6, I compare the other measures with Jaccard similarity by measuring the average change in F1-measure over only the affected instances. We see the same trends as discussed above, but difference between the measures become more pronounced.

\subsubsection{Execution Time}

As we have seen, the use of collective relational entity resolution improves entity resolution performance over attribute-based baselines. However it is more expensive computationally. Table 2.3 records the execution times in CPU seconds of the baseline algorithms and $\mathbf{C R}$ on the three datasets. All execution times are reported on a Dell Precision 870 server with $3.2 \mathrm{GHz}$ Intel Xeon processor and 3GB of memory. Let us first consider the execution times for CiteSeer and arXiv. As expected, CR takes more time than the baseline but it is still quite fast. It takes less than 3 secs for the 2,982 references in CiteSeer and less than 5 minutes for the 58,515 references in arXiv. This is around 9 times as long as the baseline for CiteSeer and 17 times for arXiv. Recall that the complexity of neighborhood similarity is linear in the average connectivity between similar names. The average number of neighbors per entity for CiteSeer is 2.15 and for arXiv it is 4.5 . So this difference in the degree of relational connectivity explains the difference in execution times for the two datasets. Also, the available attribute for these two datasets is the author 
Table 2.3: Execution times of different algorithms in CPU seconds

\begin{tabular}{|l|c|c|c|}
\hline & CiteSeer & arXiv & BioBase \\
\hline \hline A & 0.1 & 11.3 & 3.9 \\
NR & 0.1 & 11.5 & 19.1 \\
CR & 2.7 & 299.00 & 45.6 \\
\hline
\end{tabular}

name and the average number of authors per publication is very small (1.9) for both. So very little extra computation is needed for the naive relational approach over the attribute baseline.

Now let us consider BioBase. The time recorded for BioBase in Table 2.3 is not for cleaning the entire dataset. Rather, it is the average time for collectively resolving references with each of the 100 labeled names. I picked each of the 100 names in the BioBase dataset and extracted all the references relevant for resolving references with that name collectively. The time recorded for BioBase in Table 2.3 is the average time taken by different algorithms to resolve these 'relevant references' for each name. The 'relevant references' for each name are found iteratively by including all references that are reachable from the 'base references' that have this name in $k$ steps. The average number of relevant references for each of the 100 instances in 5,510. Table 2.3 shows that the difference in execution time between CR and the baselines is smaller for BioBase. One reason for this is that BioBase has many attributes in addition to author name that the attribute-only baseline also need to take into account. Also, the average number of authors per publication is 5.3 for BioBase as compared to 1.9 for the other two datasets. This makes the naive relational approach significantly more expensive than the attribute-only baseline. 


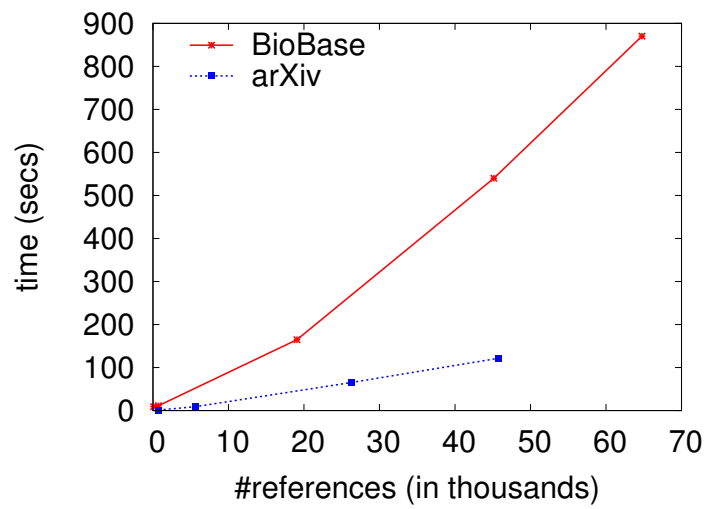

Figure 2.7: Execution time of $\mathrm{CR}$ with increasing number of references

I also used this iterative setup to explore how the collective relational entity resolution algorithm scales with increasing number of references. I created 4 datasets of varying sizes from arXiv and BioBase. Figure 2.7 shows how CR scales linearly with increasing number of references in the dataset. Recall that the complexity of $\mathbf{C R}$ is $O(n k \log n)$ for $n$ input references where $k$ represents the degree of connectivity among the references.

\subsubsection{Experiments on Synthetic Data}

As we saw in the previous section, the benefit of using collective relational entity resolution varied across the different datasets. I attribute this performance difference to the differences in structural properties of the datasets, such as the fraction of references that are ambiguous, the number of neighbors per entity, etc. To better understand how these different structural characteristics affect the performance of collective relational entity resolution, I also experiment with synthetically generated data. I explain the synthetic data generation process in Appendix A. Using this generator, I can control the different structural characteristics, such as 


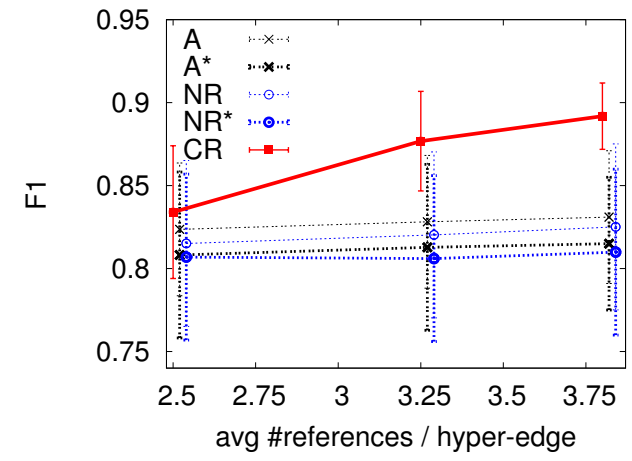

(a)

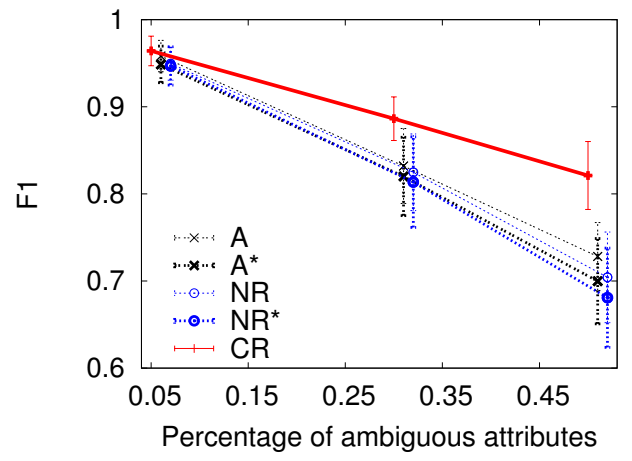

(b)

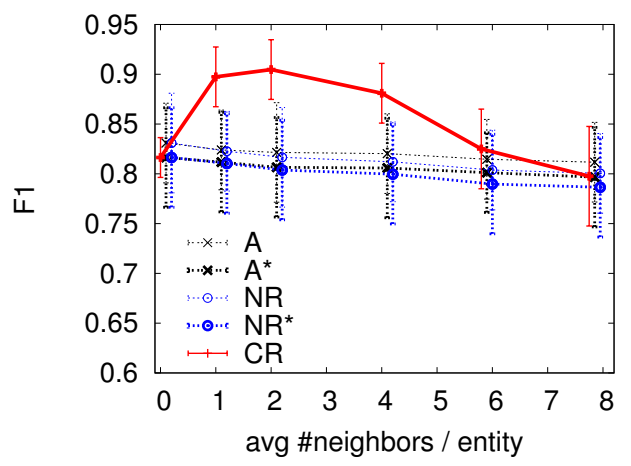

(c)

Figure 2.8: Performance of different entity resolution algorithms on data synthetically generated by varying different structural parameters such as (a) the average size of hyper-edges, (b) the percentage of ambiguous references and (c) the average number of neighbors per entity. Standard deviations are shown using error bars.

the average number of references in each hyper-edge, the percentage of ambiguous references in the data and the density of relationships among the underlying entities. As I explain in Appendix A, the synthetic data generator is not tailored solely to bibliographic data, but can model general relationships between entities, as in social network data or email data.

I performed three sets of experiments on synthetically generated data. In all of the experiments I consider average performance over 200 different runs. In the 
first experiment, I studied the effect of the number of references in each hyper-edge. The objective of this experiment is two-fold. Consider a collaborative graph, where an entity $e$ has many neighbors. If hyper-edges are small in size then two hyperedges involving a references $r_{1}$ and $r_{2}$ corresponding to $e$ may not have any other entities in common. Then it is not possible to identify $r_{1}$ and $r_{2}$ even using relational similarity. Secondly, in ambiguous domains, a single shared neighbor may not be enough to distinguish between two entities. In both cases, collective resolution is expected to benefit from larger hyper-edge sizes. In each run for this experiment, I first created an entity graph by adding 100 entities and 200 binary relationships. Then I created different reference datasets, each with 500 hyper-edges. I varied $p_{c}$ which led to different number of references in the edges. Figure 2.8(a) shows the performance of the different entity resolution algorithms on these datasets. We see that while the performances of the attribute baselines ( $\mathbf{A}$ and $\mathbf{A}^{*}$ ) does not change, the performance of $\mathbf{C R}$ improves with increasing number of references per hyperedge. Interestingly, performance of the naive relational approach $\left(\mathbf{N R}^{*}\right)$ degrades with increasing number of references. This demonstrates the importance of resolving related names instead of considering their attribute similarities only.

In the second experiment, I varied the number of ambiguous references in the data. Collective resolution is particularly useful for ambiguous references. It may be possible to address the problem of identifying dispersed references for any entity by using a smaller similarity threshold with the attribute-only baseline. In contrast, disambiguation cannot be done using attributes but is often possible using relationships. So I expected the collective resolution approach to show larger improvements 
over the baseline for more ambiguous data. I created five sets of datasets, each with 100 entities, but with different ambiguous attribute probability $p_{a}$. Then I added 200 binary relations between these entities and generated 500 hyper-edges with an average of 2 references per hyper-edge. Figure 2.8(b) compares entity resolution performance for the different algorithms on the datasets. As expected, the performance of all algorithms drops with increasing percentage of ambiguous references. However, the performance drop for $\mathbf{C R}$ is significantly slower than those for the attribute and naive relational baselines since the entity relationships helps to make the algorithm more robust. As a result, the gap between $\mathbf{C R}$ and the baselines increases as the percentage of ambiguous references in the data increases.

In the final experiment, I explored the impact of varying the number of relationships between the underlying entities. In the extreme situation, where there are no relationships between entities, clearly no improvement can be obtained using collective resolution. At the other extreme, when all entities are connected to each other, there is no pattern in the relationships that collective resolution can exploit. The objective of this experiment was to explore how increased connectivity among entities affects collective resolution. I first created a set of 100 entities. Then I created different entity graph structures by adding different number of relations between the entities. As before, I generated 500 hyper-edges (with an average of 2 references per hyper-edge) from each of these different entity-graphs and compared performances of the different algorithms for the different datasets. The results are shown in Figure 2.8(c). First note that, as expected, the performances of the attribute baselines ( $\mathbf{A}$ and $\mathbf{A}^{*}$ ) do not change significantly since they do not depend 
on the relationships. The naive relational approaches ( $\mathbf{N R}$ and $\mathbf{N R}^{*}$ ) degrade in performance with higher neighborhood sizes, again highlighting the importance of resolving related references. The performance of $\mathbf{C R}$ increases initially as the number of relationships increases. However it peaks when the average number of neighbors per entity is around 2 and then it starts falling off. In fact, it falls below the attribute-baseline when the neighborhood size increases to 8 . This is an interesting result that shows that increasing number of relationships does not always help collective entity resolution. As more relationships get added between entities, relationship patterns between entities are less informative, and may actually hurt performance. In this experiment, the probability of ambiguous attributes $p_{a}$ was 0.3 . We observe the same trend for other values of $p_{a}$, the only change is the position of the peak. The peak occurs earlier as $p_{a}$ is increased.

\subsection{Conclusion}

In summary, the relational clustering algorithm for collective entity resolution using relations is a promising approach that improves resolution performance over attribute-based and naive relational baselines. It has several nice properties in that it is simple to understand and the incremental evidence in each iteration is easy to interpret. It can be customized for different domains by using the attribute similarity measure that works best. It is also fast, owing to efficient implementation; it can resolve databases with 60,000 references in less than 5 minutes. All of these features make it an attractive tool to use for domains that require fast resolution 
with interpretable evidence.

On the other hand, this approach has certain limitations as well. For the first limitation, recall that the similarity measure in Eqn. 2.2 involves a weighting parameter $\alpha$ for combining attribute and relational similarity. It is not clear how the optimal value for $\alpha$ should be chosen for each case and, for most of the comparisons, I consider the best F1 score over all values of $\alpha$. Figure 2.5 shows the performance for a fixed value of $\alpha$ in contrast to a different optimal value for each case. It demonstrates that there are significant performance improvements using $\mathbf{C R}$ for any value of $\alpha$ over its entire range. Recall that the similarity measure uses only attributes when $\alpha=0$ and only relations when $\alpha=1$. For CiteSeer and arXiv, performance does not vary significantly with $\alpha$. Since BioBase has much higher ambiguity in terms of attributes (many references have exactly the same name and therefore mostly the same countries, and all papers are from the same area), resolution performance improves with increasing $\alpha$.

Secondly, as with any clustering algorithm, determination of the termination threshold is an issue. Note that this comes up for all of the baselines as well, and here I report best accuracy over all thresholds. This is an area of ongoing research. One other issue with the agglomerative clustering approach is that it is greedy in terms of selecting clusters to merge and the final solution would be different for a different merge sequence. Also, this approach is monotonic in that clusters only merge. Two clusters once merged cannot split back later to account for any new evidence that might be discovered. I address these issues with the probabilistic generative model, which I describe in the next chapter. 


\section{Chapter 3}

\section{A Latent Dirichlet Model for Unsupervised Entity Resolution}

In this chapter, I introduce a non-parametric probabilistic approach for solving the collective entity resolution, that addresses some of the limitations of the relational clustering approach. This chapter is organized as follows. First, I present a motivating example in Section 3.1. In Section 3.2, I first adapt the LDA model for document authors and extend it for entity resolution in Section 3.3. The sampling framework for inference is presented in Section 3.4. In Section 3.5 and Section 3.6, I describe how entity attributes are modeled. Section 3.7 describes a novel algorithm for determining the number of entities and in Section 3.8 and Section 3.9 I explore parameter choices and algorithmic improvements . Finally, I present experimental results on real and synthetic data in Section 3.10 and conclude in Section 3.11.

\subsection{A Motivating Example}

In this section, I introduce a concrete bibliographic example to explain the entity resolution problem for authors and motivate the proposed approach. Consider as an example six real paper citations P1 through P6 from CiteSeer:

- P1: "JOSTLE: Partitioning of Unstructured Meshes for Massively Parallel

Machines" C. Walshaw, M. Cross, M. G. Everett, S. Johnson 
- P2: "Partitioning Mapping of Unstructured Meshes to Parallel Machine Topologies", C. Walshaw, M. Cross, M. G. Everett, S. Johnson, K. McManus

- P3: "Dynamic Mesh Partitioning: A Unified Optimisation and Load-Balancing Algorithm", C. Walshaw, M. Cross, M. G. Everett

- P4: "Code Generation for Machines with Multiregister Operations", Alfred V. Aho, Stephen C. Johnson, Jefferey D. Ullman

- P5: "Deterministic Parsing of Ambiguous Grammars", A. V. Aho, S. C. Johnson, J. D. Ullman

- P6: "Compilers: Principles, Techniques, and Tools", A. Aho, R. Sethi, J. Ullman

Each of the 6 papers has its own author references. For instance, the first paper P1 has four references 'C. Walshaw', 'M. Cross', 'M. G. Everett' and 'S. Johnson'. In all we have 21 references in the 6 papers. The goal is to find out how many different author entities these references correspond to and which reference maps to which entity. Ground truth tells us that all of the Aho's map to the same author entity, as do the Everret's and the Ullman's. The interesting case here is that of Johnson. The four Johnson references correspond to two Johnson entities: those in papers P4 and P5 correspond to Stephen C. Johnson from Bell Labs, while those in papers P1 and P2 map to Steve P. Johnson from University of Greenwich, London. However, going by just the names of the references it is not clear why 'Stephen C. Johnson' is not 'S. Johnson', when 'Alfred V. Aho' is the same as 'A. Aho'. The 

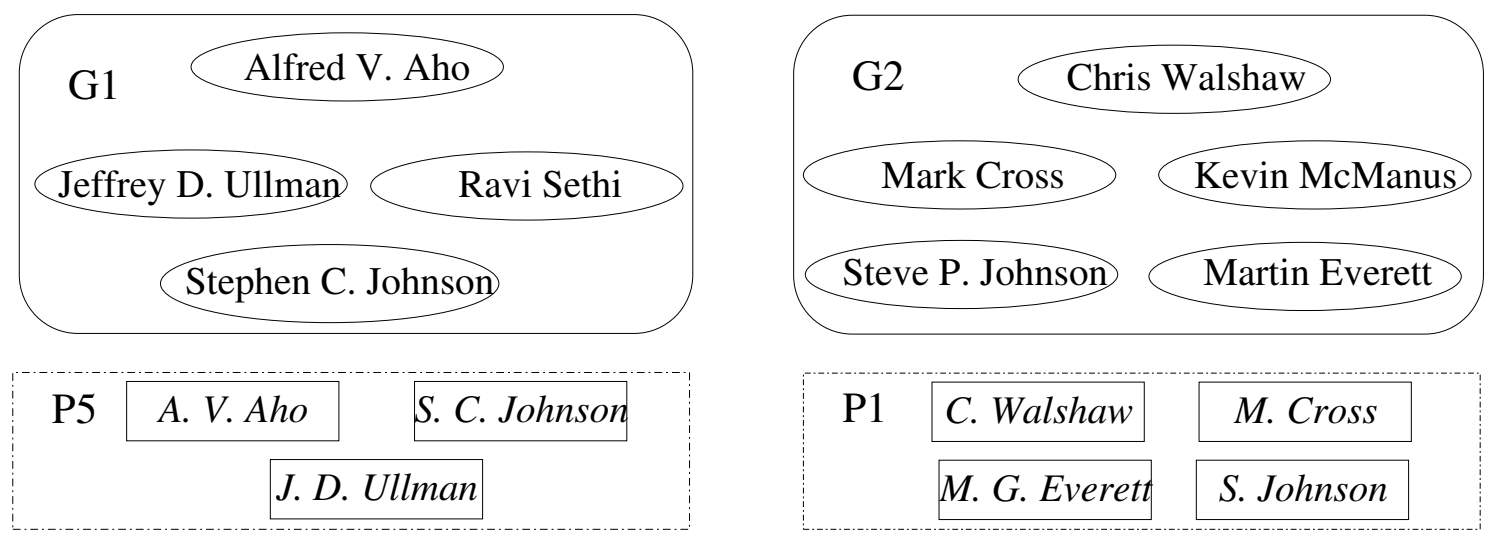

Figure 3.1: Author entities in two different collaboration groups and two generated papers. The ovals are the entities belonging to groups shown as encapsulating rectangles. Dotted rectangles represent papers with author references shown as smaller solid rectangles. Each paper is generated by the group above it.

goal will be to make use of the collaboration relationships to make these contrasting inferences simultaneously. We would like to be able to infer from the collaborations that there are two different collaboration groups in this example and authors are more likely to publish with other authors from the same group. As illustrated in Fig. 3.1, the first group G1 has Aho, Ullman and Sethi as member authors. The other group G2 has Walshaw, Cross, Everett and McManus. Stephen C. Johnson is associated with the first collaboration group, while S Johnson from papers P1 and P2 is a different person since he is associated with the second collaboration group.

In order to make these inferences, the model introduces an entity label and a group label for each reference, both of which are hidden and need to be inferred. The inference procedure is collective in that they cannot be made independently for each reference - their relationships to other references need to be considered as well. Also, the group and the entity labels are inter-dependent. The entity labels for the two Johnson's depend on their group labels, as we just saw. Also, the group 
labels depend on the entity labels in turn. Sethi from paper P6 and Johnson from paper P5 belong to the same group since they are tied by the identical entity labels for the Aho's and Ullman's in the two papers. These two hidden variables are the key distinctions of the model in comparison to some other recent ones that have been proposed. Most other approaches introduce a decision variable for each potential duplicate pair to infer whether or not they correspond to the same entity, while I introduce two variables for each reference in the data. As data sizes grow, I believe that this distinction has a significant impact.

It is interesting to note the role of papers $\mathrm{P} 3$ and $\mathrm{P} 6$ in this collective inference for the Johnson's though none of them contain a Johnson reference. They help to reinforce our belief that there are two distinct tightly knit groups or communities where member authors collaborate strongly with each other. Observe that frequent collaborations between Walshaw and Aho, and Everett and Ullman for example would have the opposite effect. Then we would think there is one collaboration group, as opposed to two, and therefore all Johnson's are more likely to be the same author.

Not surprisingly, inferring the entity labels exactly turns out to be intractable. For this model, I propose an effective Gibbs sampling approach for approximate inference. Also, one critical aspect of the inference procedure is discovering the likely number of entity labels, since the actual entities are hidden from us. I show how the number of entities can be inferred as well.

Though I use the bibliographic domain of papers and authors, the model is applicable in a straight-forward manner for other domains where noisy references to 
person entities are observed together. Examples include names of people traveling together on the same flight, names appearing together in the same email or groups of people attending the same meeting. Furthermore, this approach can be generalized to model other resolution problems. A very similar model may be used for word sense resolution in natural language documents, where the references are word occurrences and the senses are the entities to be resolved.

\subsection{LDA Model for Authors}

The idea of groups has been used for probabilistic modeling of natural language documents. Most commonly, documents are viewed as bags of words and the probabilistic approaches aim to represent the documents as mixtures over underlying 'topics', which can be imagined as probabilistic groups of semantically related words. This is very similar to the idea of modeling groups of underlying entities allowing entities that belong to the same group to co-occur in the data.

The Latent Dirichlet Allocation (LDA) model was proposed by Blei et. al. [21] as a mixture model for textual documents. In essense, it is a three level model where each document (or, co-occurrence relation, in our terminology) is modeled as a mixture over topics (or, groups, in our discussion) and then each word for that document is generated in two steps, by first sampling a topic and then a word from that topic. Though performing exact inference is not tractable in this model, it is a popular approach for group modeling topics in machine learning literature. Many authors have built on it since $[93,71]$ and efficient strategies for approximate 


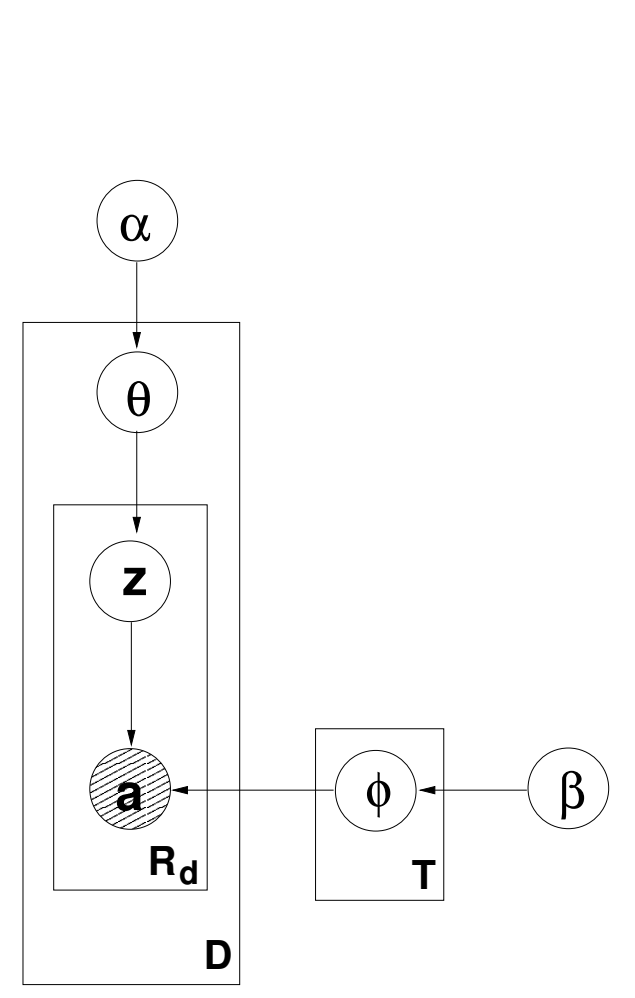

(a)

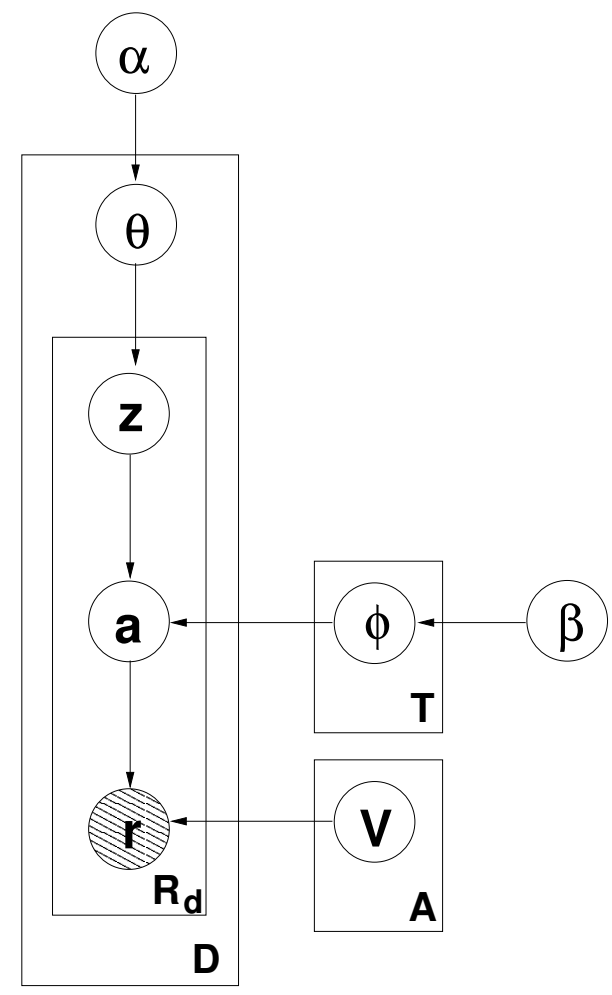

(b)

Figure 3.2: Plate representation for (a) group mixture model for authors and (b) group mixture model for author resolution from ambiguous references. Observed variables are shaded.

inference have also been proposed $[21,51,77]$.

In this section, I adapt the LDA model to a group mixture model for author entities. In this chapter will slightly modify the notation to suit the context. Entities are authors here, so I use the symbol $a$ instead of $e$ to denote author entities. Also, since each document acts as a link between its co-author references, I will use the symbol $d$ instead of $h$ for (hyper) link labels.

I start with the simple case where there is no ambiguity in the author references. In the next section, I will expand the model to handle ambiguous author references and propose inference algorithms suited to the new model. 
Consider a collection of $D$ documents and a set of $A$ authors corresponding to the authors of the documents. We have a set of $\mathrm{R}$ author references, $\left\{a_{1}, \ldots, a_{R}\right\}$. Each document can have multiple authors and for now, I assume the authors of each document are observed. For an author reference $a_{i}$, I use $d_{i}$ to denote the document in which it occurs. Further I introduce the notion of collaborative author groups. These are groups of authors which tend to co-author together. I will assume that there are $T$ different groups. Each author reference $a_{i}$ has an associated group label $z_{i}$ that takes values from 1 through $T$.

The probabilistic model is given using plate notation in Figure 3.2(a). The probability distribution over authors for each group is represented as a multinomial with parameters $\phi^{j}$, so the probability $P(a=i \mid z=j)$ of the $i^{\text {th }}$ author in the database being chosen for the $j^{\text {th }}$ group is $\phi_{i}^{j}$. We have $T$ different multinomials, one for each group. Each paper $d$ is modeled as a mixture over the $T$ groups. The distribution used is again a multinomial with parameters $\theta^{d}$, so the probability $P_{d}(z=j)$ of the $j^{\text {th }}$ group being chosen for document $d$ is $\theta_{j}^{d}$. Each $\theta^{d}$ is drawn from a Dirichlet distribution with hyperparameters $\alpha$; similarly each $\phi^{j}$ is drawn from a Dirichlet distribution with hyperparameters $\beta$.

\subsection{LDA Model for Author Resolution}

In the previous section, I assumed that the author identity can be determined unambiguously from each author reference. However, when we are dealing with author names, this is typically not the case. The same author may be represented 
in a variety of ways: 'Alfred V. Aho', 'Alfred Aho', 'AV Aho', etc. There may be mistakes due to typos or extraction errors. Finally, two 'S. Johnson's may not refer to the same author entity. One may refer to 'Stephen C. Johnson' and another may refer to 'Steve P. Johnson'. The result is that we are no longer sure of the mapping from the author reference to the author entity. We must resort to inference to identify the true author for each reference.

To capture this, I will associate an attribute $v_{a}$ with each author $a$. In addition, I add an extra level to the model that probabilistically modifies the author attributes $V_{a}$ to generate the references $\mathbf{r}=\left\{r_{1}, r_{2}, \ldots, r_{R}\right\}$. Each reference is generated by first sampling a group $z$ and then an author entity $a$ as before. Then, the author reference $r$ is generated from $a$ by modifying the attribute $v_{a}$ according to a noise model $\mathcal{N}$. I use a relatively sophisticated noise model that I explain in Section 3.6. The probability of generating an author reference $r$ from a particular author entity is defined as $P\left(r \mid v_{a}\right)$. The conditional probabilities for each reference are normalized to sum to 1 over all author entities. It is the reference $r$ that is observed, while the entity $a$ and group label $z$ are hidden variables. The LDA-ER model is represented in Figure 3.2(b).

Illustrating this in the context of our motivating example in Fig. 3.1, we have already seen how the three author entities are chosen for paper P1. The attributes $v_{a}$ for the three authors are 'Alfred V. Aho', 'Stephen C. Johnson' and 'Jeffrey D. Ullman'. However the complete/correct names do not always appear in papers or citations. In this case, the noise process modifies the attributes of the three selected entities to generate 'A. V. Aho', 'S. C. Johnson' and 'J. D. Ullman' as the three 
author references in the paper.

The probability of generating the attributes $\mathbf{r}$ for the set $\mathbf{r}$ of references for a corpus given parameters $\alpha, \beta$ and $\mathbf{V}$ can be expressed as

$$
\begin{aligned}
P(\mathbf{r} ; \alpha, \beta, \mathbf{V}) & =\prod_{d} P\left(\mathbf{r}_{d} ; \alpha, \beta, \mathbf{V}\right) \\
& =\prod_{d} \sum_{\mathbf{a}_{d}} P\left(\mathbf{r}_{d} \mid \mathbf{a}_{d} ; \mathbf{V}\right) P\left(\mathbf{a}_{d} ; \alpha, \beta\right) \\
& =\int_{\phi} P(\phi ; \beta) \prod_{d} \sum_{\mathbf{r}_{d}} P\left(\mathbf{r}_{d} \mid \mathbf{a}_{d} ; \mathbf{V}\right) \int_{\theta} P(\theta ; \alpha) P\left(\mathbf{a}_{d} \mid \theta, \phi\right) d \theta d \phi \\
& =\int_{\phi} P(\phi ; \beta) \prod_{d} \int_{\theta} P(\theta ; \alpha) \prod_{r \in d} \sum_{a} P\left(r \mid v_{a}\right) P(a \mid \theta, \phi) d \theta d \phi \\
& =\int_{\phi} P(\phi ; \beta) \prod_{d} \int_{\theta} P(\theta ; \alpha) \prod_{r \in d} \sum_{a} P\left(r \mid v_{a}\right) \sum_{j} P(z=j \mid \theta) P\left(a \mid \phi^{j}\right) d \theta d \phi \\
& =\int_{\phi} P(\phi ; \beta) \prod_{d} \int_{\theta} P(\theta ; \alpha) \prod_{r \in d} \sum_{a} P\left(r \mid v_{a}\right) \sum_{j} \prod_{i}\left(\theta_{j} \phi_{i}^{j}\right)^{\delta_{i}(a)} d \theta d \phi
\end{aligned}
$$

where $\delta_{i}(a)$ is 1 if $a=i$ and 0 otherwise.

\subsection{Inference using Gibbs Sampling}

In general, the integral in Eq. (3.1) is intractable due to coupling between $\theta$ and $\phi$. Different approximations have been proposed, including variational methods [21], Gibbs Sampling [51] and Expectation Propagation [77].

I follow the approach proposed by Griffiths and Steyvers [51] where $\theta$ and $\phi$ are not directly estimated as parameters. Instead, the posterior distribution $P(\mathbf{z}, \mathbf{a} \mid \mathbf{r})$ is first constructed and then $\theta$ and $\phi$ are estimated from this posterior distribution. Now, the joint probability can be derived from Eq. (3.1) as:

$$
P(\mathbf{z}, \mathbf{a}, \mathbf{r})=P(\mathbf{z}) P(\mathbf{a} \mid \mathbf{z}) P(\mathbf{r} \mid \mathbf{a})
$$


where

$$
P(\mathbf{z})=\left(\frac{\Gamma(T \alpha)}{\Gamma(\alpha)^{T}}\right)^{D} \prod_{d=1}^{D} \frac{\prod_{t} \Gamma\left(\alpha+C_{d t}^{D T}\right)}{\Gamma\left(T \alpha+C_{d *}^{D T}\right)}
$$

is the probability of the joint group assignment to all references and

$$
P(\mathbf{a} \mid \mathbf{z})=\left(\frac{\Gamma(A \beta)}{\Gamma(\beta)^{A}}\right)^{T} \prod_{t=1}^{T} \frac{\prod_{a} \Gamma\left(\beta+C_{a t}^{A T}\right)}{\Gamma\left(A \beta+C_{* t}^{A T}\right)}
$$

is the conditional probability of the references given the groups and

$$
P(\mathbf{r} \mid \mathbf{a})=\prod_{i=1}^{R} P\left(r \mid v_{a_{i}}\right)
$$

is the conditional probability of the references given the authors. $C_{d t}^{D T}$ is the number of times group $t$ has been observed for the references in document $d$ and $C_{d *}^{D T}=$ $\sum_{t} C_{d t}^{D T}$. Similarly, $C_{a t}^{A T}$ is the number of times references to author $a$ have been observed with group label $t$ in all documents.

I construct a Markov chain that converges to the posterior distribution $P(\mathbf{z}, \mathbf{a} \mid$ r) and then draw samples from this Markov chain. Each state in the Markov chain is an assignment of a group label and an author label to all $R$ references. In the Gibbs Sampling approach, the labels for each reference are sequentially sampled conditioned on the current labels of all other references. By construction, this Markov chain converges to the target posterior distribution. However, I first need to define the full conditional distribution $P\left(z_{i}=t, a_{i}=a \mid \mathbf{z}_{-i}, \mathbf{a}_{-i}, \mathbf{r}\right)$, where $\mathbf{z}_{-i}$ is the set of all but the $i^{\text {th }}$ group label and $\mathbf{a}_{-i}$ all but the $i^{\text {th }}$ author label. In words, this is the probability that the $i^{\text {th }}$ reference comes from the $t^{\text {th }}$ group considering the current group and author assignment to all other references. 
I derive this full conditional distribution as

$$
P\left(z_{i}=t, a_{i}=a \mid \mathbf{z}_{-i}, \mathbf{a}_{-i}, \mathbf{r}\right) \propto \frac{C_{(-i) d_{i} t}^{D T}+\alpha}{C_{(-i) d_{i} *}^{D T}+T \alpha} \frac{C_{(-i) a t}^{A T}+\beta}{C_{(-i) * t}^{A T}+A \beta} P\left(r_{i} \mid v_{a}\right)
$$

The factorization makes intuitive sense. The first term is the probability of group $t$ in document $d_{i}$, the second is the probability of author $a$ in group $t$ and the third is the probability of the author attribute $v_{a}$ being corrupted into the $i^{\text {th }}$ reference attribute.

Instead of sampling $z_{i}$ and $a_{i}$ as a block, they can be sampled separately:

$$
\begin{gathered}
P\left(z_{i}=t \mid \mathbf{z}_{-i}, \mathbf{a}, \mathbf{r}\right) \propto \frac{C_{(-i) d_{i} t}^{D T}+\alpha}{C_{(-i) d_{i} *}^{D T}+T \alpha} \frac{C_{(-i) a_{i} t}^{A T}+\beta}{C_{(-i) * t}^{A T}+A \beta} \\
P\left(a_{i}=a \mid \mathbf{z}, \mathbf{a}_{-i}, \mathbf{r}\right) \propto \frac{C_{(-i) a t_{i}}^{A T}+\beta}{C_{(-i) * t_{i}}^{A T}+A \beta} P\left(r_{i} \mid v_{a}\right)
\end{gathered}
$$

\subsection{Modeling Author Attributes}

In the previous section, I assumed that the author attribute values $v_{a}$ are known. But in general, the author attributes will not be known and will need to be inferred from the references. The conditional distribution for sampling groups $z_{i}$ is not directly affected by the attributes. However, the attributes influence the assignment of author labels $a_{i}$, since a reference $r_{i}$ is more likely to be assigned to an author with similar attributes. Conversely, any author attribute $v_{i}$ depends on the

references that have author label $i$. Incorporating a prior $P(\mathbf{v})=\prod_{i=1}^{A} P\left(v_{i}\right)$ into the joint distribution in Eq. (3.2), I derive the conditional distribution for assigning 
a value $v$ to $v_{i}$ given all author labels and references as:

$$
P\left(v_{i}=v \mid \mathbf{a}, \mathbf{r}\right) \propto P(v) \prod_{j=1}^{R} P\left(r_{j} \mid v\right) \delta_{i}\left(a_{j}\right)
$$

Intuitively, $v_{i}$ should be set to the most likely value that explains the generation of the references assigned to author $i$. For example, if multiple "J.S. Smith" and "John Smith" references have been assigned author label $i$ along with the reference "Jhon Smth", then the author attribute $v_{i}$ is most likely to be "John S. Smith". The sampling algorithm now also samples the author attributes $v_{i}$ iteratively, conditioned on the references and current author assignments, along with sampling the group and entity labels for each reference. For 'free authors' to which no references are currently assigned, I set the attributes to a special value ' $\star$ '. In order to make the model to prefer free authors over assigned authors, I assign a higher prior probability $P(\star)$ than all other attributes.

\subsection{Noise Model}

The different ways for distorting or modifying an author attribute to a reference in a document is captured by the noise model $\mathcal{N}$. It handles first, middle and last names independently. The first name can be initialed with probability $p_{F I}$, dropped with probability $p_{F D}$ or retained as a whole with probability $p_{F R}$, where $p_{F I}+p_{F D}+p_{F R}=1$. There are similar parameters $p_{M I}, p_{M D}$ and $p_{M R}$ for the middle name. The probabilities for the first and middle initials being incorrect are $p_{F I r}$ and $p_{M I r}$. These are expected to be lower than $p_{R}$. Last names and retained first or middle names may be corrupted by characters being inserted, deleted or 
replaced with probabilities $p_{I}, p_{D}$ and $p_{R}$ respectively. The minimum numbers of insertion $\left(n_{I}\right)$, deletion $\left(n_{D}\right)$ and replacement $\left(n_{R}\right)$ operations for mutating an author attribute $v_{a}$ to a reference $r$ are obtained using edit-distance for strings. Then the mutation probability is $P\left(r \mid v_{a}\right)=p_{I}^{n_{I}} \cdot p_{D}^{n_{D}} \cdot p_{R}^{n_{R}}$.

\subsection{Determining Number of Entities}

In the development up until now, I have considered the number of authors $A$ to be given, when in practice this needs to be estimated. One of the contributions of this work is an unsupervised method for determining the number of entities. I propose a novel approach that avoids searching explicitly over the possible number of author entities and instead adapts it within the sampling framework.

\subsubsection{Basic Inference With Gibbs Sampling}

I first describe a novel but simple Gibbs sampling algorithm for iteratively sampling the values of the hidden group and entity labels for each reference conditioned on the existing labels of all other references. Equations 3.5, 3.6 and 3.5 form the basis of this algorithm. I first sample a group label for each reference according to Eq. (3.5). Next, I sample an entity label for each reference according to Eq. (3.6). The difference for the entities is that the number of entity labels is unknown and needs to be inferred by the algorithm. So I either choose an existing entity label or alternatively a hitherto unused one. For a new entity label, its observed occur-

rence count $C_{(-i) a t_{i}}^{A T}$ is 0 . But the parameter $\beta$ ensures a non-zero probability of a 
new label being chosen. Also, the attribute $v_{a}$ for a new entity is unknown. So I use a fixed value for the probability $P\left(r_{i} \mid v_{a}\right)$ for a new entity $a$ that controls how frequently new entity labels are sampled. Once all the entity labels are sampled, in the third step the attribute values are sampled for each of the existing entities according to Eq. (3.5). The iterations continue till convergence. There is a connection between this flavor of Gibbs sampling inference for number of entities and the Dirichlet process which I describe in the next subsection.

\subsubsection{Relation to the Dirichlet Process}

The Dirichlet process was introduced by Ferguson [45] and Antoniak [4] as a non-parametric statistical approach that allows the complexity of the model to grow with increasing size of the data. In the context of our application, we would like the number of entities to be inferred in model rather than it being a fixed parameter, and we would like the model to be able to accommodate a greater number of entities as the number of references in the data grows. The Dirichlet process can be imagined as a distribution over discrete distributions and is used as follows for choosing the number of components in a mixture model. A distribution (or a component) is first drawn from the Dirichlet process, the parameters are then sampled from this distribution and finally the data is drawn using these parameters. Drawing a parallel with the application here, I can sample an entity first, choose the parameters (the attribute) for that entity and then finally generate the reference using the en-

tity parameters. When the Dirichlet process is integrated out, a clustering effect is 
observed in the conditional distribution for choosing the $n^{\text {th }}$ component given $n-1$ previous component draws. The probability of choosing one of the existing components is proportional to the number of times it has been chosen in the previous $n-1$ draws, while a new component has a nonzero probability of being sampled. In particular, let $G_{0}$ be the baseline probability distribution over discrete components $\eta$ and $\alpha$ be a scalar. Then, given the $n-1$ draws $\eta_{1: n-1}$, the distribution for the $n^{t h}$ component is given by

$$
\eta_{n}=\left\{\begin{array}{lrl}
\eta_{i}^{*} & \text { with prob } & \frac{n_{i}}{n-1+\alpha} \\
\eta, \eta \sim G_{0} & \text { with prob } & \frac{\alpha}{n-1+\alpha}
\end{array}\right.
$$

where $n_{i}$ is the number of times $\eta_{i}^{*}$ has occurred in $\eta_{1: n-1}$.

Exact inference is intractable in the Dirichlet process mixture model but approximate inference techniques have been proposed [81, 19]. Of particular interest is the Gibbs sampling strategy proposed by Neal [81]. This algorithm iteratively samples the component label $a_{i}$ for the $i^{\text {th }}$ data object $r_{i}$ from the conditional distribution given the other labels:

$$
\begin{aligned}
& P\left(a_{i}=k \mid \mathbf{r}, \mathbf{a}_{-\mathbf{i}}, \alpha\right) \\
= & P\left(a_{i}=k \mid \mathbf{a}_{-\mathbf{i}}, \alpha\right) P\left(r_{i} \mid \mathbf{r}_{-\mathbf{i}}, \mathbf{a}_{-\mathbf{i}}, a_{i}=k\right)
\end{aligned}
$$

For an existing component $k$

$$
P\left(a_{i}=k \mid \mathbf{a}_{-i}, \alpha\right)=\frac{C_{(-i) k}^{A}}{\alpha+N-1}
$$

where $C_{(-i) k}^{A}$ is the number of previous assignments to the $k^{\text {th }}$ component without counting the $i^{\text {th }}$ assignment. For a component $k$ that has not been used before

$$
P\left(a_{i}=k \mid \mathbf{a}_{-i}, \alpha\right)=\frac{\alpha}{\alpha+N-1}
$$


We may imagine LDA-ER as the Dirichlet process mixture model augmented with a group structure above it that enables it to capture relations between the components or entities. In LDA-ER, a group $z_{i}=t$ is first sampled for the $i^{t h}$ reference from the distribution over groups for the document and then an entity is sampled from it. In the Dirichlet process, any previously existing entity may be chosen in this step depending on their prior counts. But in LDA-ER, the choice is controlled by the sampled group $t$. Entities that have previously been associated with this sampled group are much more likely to be chosen. This distinction allows LDA-ER to model relations between entities. As in the Dirichlet process, alternatively a new entity may be selected in LDA-ER. However, this new entity now becomes associated with group $t$ and may be chosen for future references from this group. This difference is clearly observable from the conditional distributions in Eq. (3.8) and Eq. (3.6). While the probability for choosing the $k^{\text {th }}$ entity in Eq. (3.8) depends on $C_{(-i) a}^{A}$ which is the number of previous occurrences of entity $a$, in Eq. (3.6) it

depends on $C_{(-i) a t}^{A T}$ which is the number of joint occurrences of group $t$ and entity $a$. This coupling of the group and entity labels distinguishes the LDA-ER model from the Dirichlet process mixture model.

\subsubsection{Block Assignment for Entity Resolution}

As has been noted in the case of naive Gibbs sampling for inference in the Dirichlet process mixture model [19], iteratively estimating the group and entity label for each reference separately, as described in Sec. 3.7.1 can be prohibitively 
slow. I now describe a novel algorithm that overcomes this problem by reassigning entity labels for a set of entities at the same time. This achieves an agglomerative clustering effect on the references. Observe that for any assignment of entity labels to references, each entity label defines a cluster — all references that have this entity label belong to this cluster. Sampling a new label for each reference separately is equivalent to an individual reference migrating from one cluster to another. Agglomerative clustering is significantly faster since pairs of clusters merge into one. I achieve the same effect with the new sampling algorithm that I propose. In addition, I allow existing clusters to split. The conditional probabilities for these choices for any particular entity cluster given the entity and group labels for all other references are derived from the joint distribution in Eq. (3.2). As in traditional Gibbs sampling, these probabilities then form the transition probabilities in a Markov process.

I define a cluster by picking an author label $j$ and consider the set $\mathbf{s}$ of reference indices that have $j$ as their author label: $\mathbf{s}=\left\{i \mid a_{i}=j\right\}$. I assign new author labels to all references indexed by cluster s simultaneously. In general, the number of possible author assignments to $\mathbf{s}$ is exponential in $|\mathbf{s}|$ and it is virtually impossible to enumerate all these different probabilities for sampling.

Instead, in the algorithm I restrict the space of candidates such that the cluster of references assigned to a particular author label may (a) merge with a cluster currently assigned to another author label, (b) stay unchanged or (c) split and have a part assigned to a hitherto unassigned author label $j^{\prime}$. Case (a) is similar to two author clusters merging and the number of authors is effectively decreased by one. In case (c), an author cluster splits into two and the number of authors is effectively 
increased by one. However, the number of possible partitions of $\mathbf{s}$ into $j$ and $j^{\prime}$ is still $2^{|\mathbf{s}|}$. The simple but restricted solution that I use is splitting to the set that last merged into label $j$ via option (a).

I first consider assigning a single author label to all of cluster s. The full conditional distribution I need to derive is $P\left(\mathbf{a}_{\mathbf{s}}=i \mid \mathbf{z}, \mathbf{a}_{-\mathbf{s}}, \mathbf{r}\right)$ which is the probability of all the labels $\mathbf{a}_{\mathbf{s}}$ in cluster $\mathbf{s}$ being set to $i$ conditioned on all references and group labels and all other author labels. Note that

$$
P\left(\mathbf{a}_{\mathbf{s}}=i \mid \mathbf{z}, \mathbf{a}_{-\mathbf{s}}, \mathbf{r}\right)=\frac{P\left(\mathbf{a}_{\mathbf{s}}=i, \mathbf{a}_{-\mathbf{s}}, \mathbf{z}, \mathbf{r}\right)}{P\left(\mathbf{a}_{-\mathbf{s}}, \mathbf{z}, \mathbf{r}\right)}=K \times P\left(\mathbf{a}_{\mathbf{s}}=i, \mathbf{a}_{-\mathbf{s}}, \mathbf{z}, \mathbf{r}\right)
$$

where $K$ is the same for all values of $i$. Note that all topic labels $\mathbf{z}$ and remaining author labels $\mathbf{a}_{-(\mathbf{s})}$ are fixed across all assignments $i$ to $\mathbf{a}_{\mathbf{s}}$. The full conditional distribution can be factored so that it has a term for each document, group and reference. Looking at Eqn. 3.3, we can see that the documents terms are identical for all $i$. The $C_{d t}^{D T}$ counts are the same for all group labels $t$ and documents $d$ since the all group labels are held fixed. On the other hand, the reference terms matter only for those references indexed by $s$, since they are assigned new author labels.

$$
\operatorname{term}_{R}=\prod_{j \in s} P\left(r_{j} \mid v_{i}\right)
$$

The group term is however nontrivial and needs careful consideration. From Eqn. 3.4

$$
\operatorname{term}_{G}=\prod_{t=1}^{T} \frac{\prod_{a} \Gamma\left(\beta+C_{(\mathbf{s}) a t}^{A T}+C_{(-\mathbf{s}) a t}^{A T}\right)}{\Gamma\left(A \beta+C_{(\mathbf{s}) * t}^{A T}+C_{(-\mathbf{s}) * t}^{A T}\right)}
$$

where $C_{(\mathbf{s}) a t}^{A T}$ is the number of times author $a$ and group $t$ have been jointly assigned to references in $\mathbf{s}$, and $C_{(-\mathbf{s}) a t}^{A T}$ is the number of such assignments outside $\mathbf{s}$. Let $\mathbf{z}_{\mathbf{s}}$ 
be the set of groups currently assigned to the references indexed by s. For groups $t \notin \mathbf{z}_{\mathbf{s}}$ and authors $a \neq i$, the count $C_{a t}^{(\mathbf{s}) A T}$ is 0 and the corresponding terms are independent of the assignment $i$. Therefore

$$
\begin{aligned}
\operatorname{term}_{G} & =K \prod_{t \in \mathbf{z}_{\mathbf{s}}} \frac{\Gamma\left(\beta+C_{(\mathbf{s}) i t}^{A T}+C_{(-\mathbf{s}) i t}^{A T}\right)}{\Gamma\left(A \beta+C_{(\mathbf{s}) * t}^{A T}+C_{(-\mathbf{s}) * t}^{A T}\right)} \\
& =K \prod_{t \in \mathbf{z}_{\mathbf{s}}} \frac{\left(\beta+C_{(-\mathbf{s}) i t}^{A T}+C_{(\mathbf{s}) i t}^{A T}-1\right) \ldots\left(\beta+C_{(-\mathbf{s}) i t}^{A T}\right) \Gamma\left(\beta+C_{(-\mathbf{s}) i t}^{A T}\right)}{\left(A \beta+C_{(-\mathbf{s}) * t}^{A T}+C_{(\mathbf{s}) * t}^{A T}-1\right) \ldots\left(A \beta+C_{(-\mathbf{s}) * t}^{A T} \Gamma\left(A \beta+C_{(-\mathbf{s}) * t}^{A T}\right)\right.} \\
& =K^{\prime} \prod_{t \in \mathbf{z}_{\mathbf{s}}} \frac{\left(\beta+C_{(-\mathbf{s}) i t}^{A T}+C_{(\mathbf{s}) i t}^{A T}-1\right) \ldots\left(\beta+C_{(-\mathbf{s}) i t}^{A T}\right)}{\left(A \beta+C_{(-\mathbf{s}) * t}^{A T}+C_{(\mathbf{s}) * t}^{A T}-1\right) \ldots\left(A \beta+C_{(-\mathbf{s}) * t}^{A T}\right)}
\end{aligned}
$$

Denoting

$$
\begin{gathered}
T(t, i)=\prod_{n=1}^{C_{(\mathbf{s}) i t}^{A T}}\left(\beta+C_{(-\mathbf{s}) i t}^{A T}+C_{(\mathbf{s}) i t}^{A T}-n\right) \\
T(t, *)=\prod_{n=1}^{C_{(\mathbf{s}) * t}^{A T}}\left(A \beta+C_{(-\mathbf{s}) * t}^{A T}+C_{(\mathbf{s}) * t}^{A T}-n\right)
\end{gathered}
$$

the group term can be written as

$$
\operatorname{term}_{G} \propto \prod_{t \in \mathbf{z}_{\mathbf{s}}} \frac{T(t, i)}{T(t, *)}
$$

Putting everything together, the conditional distribution can be written as

$$
P\left(\mathbf{a}_{\mathbf{s}}=i \mid \mathbf{z}, \mathbf{a}_{-\mathbf{s}}, \mathbf{r}\right) \propto \prod_{t \in \mathbf{z}_{\mathbf{s}}} \frac{T(t, i)}{T(t, *)} \prod_{j \in s} P\left(r_{j} \mid v_{i}\right)
$$

An Interpretation of Block Assignment: Here I show how the terms in this conditional probability can be rearranged so that the result makes intuitive sense. Let $j$ be an index into cluster $\mathbf{s}$ and $t_{j}$ be the group label for that reference. Also, consider cluster $\mathbf{s}$ to be an ordered set and denote by $\mathbf{s}_{<j}$ the set of elements in $\mathbf{s}$ 
strictly before position $j$. Then I can rewrite Eq. (3.11) as

$$
\begin{aligned}
& P\left(\mathbf{a}_{\mathbf{s}}=i \mid \mathbf{z}, \mathbf{a}_{-\mathbf{s}}, \mathbf{r}\right) \\
\propto & \prod_{j \in s} \frac{\beta+C_{\left(\mathbf{s}_{<j}\right) i t_{j}}^{A T}+C_{(-\mathbf{s}) i t_{j}}^{A T}}{A \beta+C_{\left(\mathbf{s}_{<j}\right) * t_{j}}^{A T}+C_{(-\mathbf{s}) * t_{j}}^{A T}} P\left(r_{j} \mid v_{i}\right)
\end{aligned}
$$

Here $C_{\left(\mathbf{s}_{<j}\right) i t}^{A T}$ is the number of times author label $i$ and group label $t$ have occurred jointly for just the references in $\mathbf{s}_{<j}$. I interpret this as follows. I assign author labels to the references in cluster $\mathbf{s}$ in sequence. For each assignment, the second term is the probability of the reference given the author and the first term is the probability of the author label for the reference given its current group label, including the assignments already made in the sequence as additional evidence. It must be stressed that this ordering is introduced solely for interpretation purposes and the actual probability is independent of the ordering. Note that Eq. (3.12) reduces to Eq. (3.6) as expected when cluster $\mathbf{s}$ has a single element.

For the case when I partition cluster $\mathbf{s}$ into $\mathbf{s}_{\mathbf{1}}$ and $\mathbf{s}_{\mathbf{2}}$ and assign two different author labels to them, the conditional probability looks very similar:

$$
\begin{aligned}
& P\left(\mathbf{a}_{\mathbf{s}_{\mathbf{1}}}=i, \mathbf{a}_{\mathbf{s}_{\mathbf{2}}}=i^{\prime} \mid \mathbf{z}, \mathbf{a}_{-\mathbf{s}}, \mathbf{r}\right) \\
\propto & \prod_{t \in \mathbf{z}_{\mathbf{s}}} \frac{T(t, i) T\left(t, i^{\prime}\right)}{T(t, *)} \prod_{j \in s_{1}} P\left(r_{j} \mid v_{i}\right) \prod_{j \in s_{2}} P\left(r_{j} \mid v_{i^{\prime}}\right)
\end{aligned}
$$

Observe that when one author label merges with another according to Eq. (3.11), the attribute of the freed author $j$ changes from $v_{j}$ to the free attribute ' $\star$ '. The difference in prior probabilities of the two attribute values leads to an additional term in the merge probability in Eq. (3.11): $P(\star) / P\left(v_{j}\right)$. Similarly, when splitting the references assigned to author $j$ between $j$ and currently unassigned $j^{\prime}$, the attribute 
of author $j^{\prime}$ changes to $v_{j^{\prime}}$ from ' $\star$ ' and the split probability has the additional term $P\left(v_{j^{\prime}}\right) / P(\star)$. Therefore, the higher the prior probability of ' $\star$ ' relative to other attributes, the higher will be the likelihood of a merge compared to a split.

Putting everything together, the entity resolution algorithm starts from an initial assignment of authors and groups to all references and iterates over three steps sequentially until convergence. First, it samples a group label for each reference. This has complexity $O(R T)$ for $R$ references and $T$ group labels. Then for each assigned author label, it samples the next author label for its current references. This requires $O(A S)$ operations for $A$ author labels and a maximum of $S$ potential duplicates per author. Finally, it samples an attribute for each assigned author label, requiring $O(A)$ operations. For each round of sampling authors and attributes, I do several iterations of group sampling to let the group labels stabilize for the current author assignments. Note that all stages in an iteration are linear in the number of references and author labels allowing the model to scale to large datasets as I demonstrate in the experimental section.

\subsection{Determining Model Parameters}

I have described how the numbers of authors can be determined within the sampling procedure. The remaining aspects of the model are the number of groups and the Dirichlet hyper-parameters. Their choice affects performance in different ways. 


\subsubsection{Number of Groups}

I begin by observing that the choice of the number of groups is subjective and not as critical as the number of entities. Relationships among the same set of entities can be captured with different number of groups at different levels of resolution. While it is possible to estimate the likely number of groups from the data, it is an area of potential future research. Here I consider the effect of varying number of groups on entity resolution. Recall that our guiding intuition is to assign the same author label to sets of references when they are similar and have similar group distributions. When the number of groups $T$ is too small, misleading similarities in group distributions are likely to be observed, leading to false positives. If $T$ is too

high, references to the same author can get split over different groups, making false negatives likely. In other words, lower $T$ favors higher recall and lower precision, while higher $T$ leads to lower recall with higher precision.

\subsubsection{Hyper-parameters}

To appreciate the roles of $\alpha$ and $\beta$, note from Eq. (3.5) that when $\alpha=0$, a reference is forced to pick a group label from the other references in the same document. Similarly, when $\beta=0$, a reference has to pick a group label from other references to the same author, and also an author label from other references with the same group label. In general, for low values of $\alpha$ and $\beta$, the model tends to overfit the data. This is particularly undesirable for entity resolution, since I need to estimate the number of authors and need to generalize from the current author 
assignments. To get a feel for what values are appropriate, observe that $T \alpha$ is the number of pseudo reference counts added to each document. Since in most cases documents will have one or two authors, I set $T \alpha$ to be 0.25 . Similarly, $A \beta$ is the number of pseudo references for each topic. I set $\beta$ according to the number of references in the dataset and the number of topics. A typical value for $A \beta$ is 5 .

\subsubsection{Noise Model Parameters}

I iteratively estimate the noise parameters from data in a unsupervised manner. I start from an initial estimate that is typical of some datasets I explored. For instance, first names are initialed and dropped with probabilities 0.75 and 0.001 (0.25 and 0.7 for middle names) and is incorrect with probability 0.0005 (0.001 for middle names). Characters may be dropped, replaced or inserted, each with probability 0.0025. After every author sampling step, I re-estimate the probabilities looking at each reference attribute and the attribute of the author it has been assigned to. However, the estimates from the initial iterations may not be good. For example, when all references are distinct entities, all corruption probabilities are estimated to be 0 . To prevent this, estimates are made to evolve slowly. A weighted combination of the current probabilities and the new estimates yields the probabilities for the next iteration. Typically, I retain current estimates with weight 0.9. 


\subsection{Algorithm Refinements}

Unlike group labels, author labels for references are sampled from a restricted space. Here I propose improvements for the sampling algorithm for inferring the author labels.

\subsubsection{Bootstrapping Author Labels}

Initialization of author labels is an issue both for convergence time and quality. One option is to assign the same initial label to any two references that have attributes $v_{1}$ and $v_{2}$, where either $v_{1}=v_{2}$ or $v_{1}$ is an initialed form of $v_{2}$. However, for domains where last names repeat very frequently, like Chinese, Japanese or Indian names, this can affect the initial accuracy quite adversely, from which it is hard to recover. For the case of such common last names ${ }^{1}$, I propose an improved bootstrapping scheme. I assign the same author label to pairs only when they have document co-authors with the same initial author label. This improves bootstrap accuracy significantly for one of the datasets that has frequently repeating names.

\subsubsection{Group Evidence for Author Self Loops}

Recall that Eq. (3.10) shows the group evidence for different transitions for cluster s. $C_{(-\mathbf{s}) a t}^{A T}$ is the number of references outside cluster s that have author label $a$ and group label $t$. For any group $t$, it is the group evidence for merging with the cluster for author label $a$. However, if $\mathbf{s}$ is the cluster of references with author level

\footnotetext{
${ }^{1}$ http://en.wikipedia.org/wiki/List_of_most_popular_family_names
} 
$j$, then $C_{(-\mathbf{s}) j t}^{A T}$ will be 0 for all group labels $t$, since there are no references outside cluster $\mathbf{s}$ with author label $j$. Therefore, cluster $\mathbf{s}$ has little affinity to itself when considering group evidence and prefers merging with other clusters. Note however that every cluster has higher attribute affinity to itself than to other clusters. I introduce a scalar parameter that allows us to have additional control on the rate of cluster merges. I consider a small fraction $\delta$ of $C_{(\mathbf{s}) j t}^{A T}$ as external group evidence for $j$. The higher the value of $\delta$, the stronger has to be the evidence to cause an existing author label to merge with another label or to split into two.

\subsection{Experimental Evaluation}

I begin by evaluating the algorithm on two real citation datasets. I compare the collaborative entity resolution model (LDA-ER) with the best attribute-based models. Next, to gain further understanding of the conditions under which entity resolution benefits from collaborative group information, I evaluate the proposed model on a broad range of synthetic datasets with varying relational structure.

\subsubsection{Results on Citation Data}

I first perform experimental evaluations on two citation datasets, which I have already introduced in Section 2.6. The first is the CiteSeer dataset containing citations to papers from four different areas in machine learning, originally created by Giles et al. [49]. This has 2,892 references to 1,165 authors, contained in 1,504 documents. The second dataset is significantly larger; arXiv contains papers from 
high energy physics used in KDD Cup $2003^{2}$. This has 58,515 references to 9,200 authors, contained in 29,555 papers.

To evaluate the algorithms, I measure the performance of the models for detecting duplicates in terms of precision, recall and $F 1$ on pairwise duplicate decisions. It is practically infeasible to consider all pairs, particularly for arXiv, so as others have done, I employ a 'blocking' approach to extract the potential duplicates. This approach retains $\sim 99 \%$ of the true duplicates for both datasets.

I use a simple scheme for attribute priors, where common last names are set to be 10 times more likely than other last names, and the free attribute ' $\star$ ' is 10 times more likely than common names. When sampling group labels given the entity assignments at each step, I iterate until the log-likelihood converges. Typically for the first few steps, I perform 50 group sampling iterations for each author iteration. Thereafter I proceed with 20 group iterations for each author iteration. The $F 1$ converges in about 30 author iterations for CiteSeer and 50 author iterations for arXiv. On a 3.2GHz Dell Precision 670 Intel Xeon server, this takes between 2.5 and 10 minutes for CiteSeer and between 2 and 12 hours for arXiv depending on the number of groups. As discussed in Section 3.9.2, I use a small fraction $(\delta=0.5 \%)$ of group evidence for self probabilities.

As a baseline (A), I compare with the hybrid SoftTF-IDF measure [30] that has been shown to outperform other unsupervised approaches for text-based entity resolution. Essentially, it augments the TF-IDF similarity for matching token sets with approximate token matching using a secondary string similarity measure. Jaro-

\footnotetext{
${ }^{2}$ http://www.cs.cornell.edu/projects/kddcup/index.html
} 
Winkler is reported to be the best secondary similarity measure for SoftTF-IDF. I also experiment with the Jaro and the Scaled Levenstein measures. However, directly using an off-the-shelf string similarity measure for matching names results in very poor recall. From domain knowledge about names, I know that first and middle names may be initialed or dropped. A black-box string similarity measure would unfairly penalize such cases. To deal with this, A uses string similarity only for last names and retained first and middle names. In addition, it uses drop probabilities $p_{D r o p F}$ and $p_{D r o p M}$ for dropped first and middle names, initial probabilities $p_{F I}$ and $p_{M I}$ for correct initials and $p_{F I r}$ and $p_{M I r}$ for incorrect initials. The probabilities I used are $0.75,0.001$ and 0.001 for correctly initialing, incorrectly initialing and dropping the first name, while the values for the middle name are $0.25,0.7$ and 0.002. I calculated the probabilities from the labeled datasets and then hand-tuned them for performance. The observation is that baseline resolution performance does not vary significantly as these values are varied over reasonable ranges.

A only reports pairwise match decisions, which are often inconsistent globally. I also evaluate a second baseline $\mathbf{A}$ * which takes a transitive closure over the pairwise decisions in $\mathbf{A}$. Both $\mathbf{A}$ and $\mathbf{A}^{*}$ need a similarity threshold for deciding duplicates and determining the right threshold is a problem for these algorithms. One of the strengths of LDA-ER is that it does not require any similarity threshold. For comparison, I consider the best $F 1$ that can be achieved by the baselines over all thresholds.

Table 3.1 records baseline performance with various string similarity measures coupled with SoftTF-IDF. Note that the best baseline performance is with Jaro 
Table 3.1: Performance of $\mathbf{A}$ and $\mathbf{A} *$ in terms of $F 1$ using various secondary similarity measures with SoftTF-IDF. The measures compared are Scaled Levenstein (SL), Jaro (JA), JaroWinkler (JW) and the generative similarity model used with LDA-ER (Gen).

\begin{tabular}{|l|cccc|cccc|}
\hline & \multicolumn{4}{|c|}{ CiteSeer } & \multicolumn{4}{c|}{ arXiv } \\
& SL & JA & JW & Gen & SL & JA & JW & Gen \\
\hline \hline A & 0.980 & $\mathbf{0 . 9 8 1}$ & 0.980 & 0.982 & $\mathbf{0 . 9 7 6}$ & 0.976 & 0.972 & 0.975 \\
A $^{*}$ & 0.989 & $\mathbf{0 . 9 9 1}$ & 0.990 & 0.990 & $\mathbf{0 . 9 7 1}$ & 0.968 & 0.965 & 0.970 \\
\hline
\end{tabular}

as secondary string similarity for CiteSeer and Scaled Levenstein for arXiv. It is also worth noting that a baseline without initial and drop probabilities scores below 0.5 F1 using Jaro and Jaro-Winkler for both datasets. It is higher with Scaled Levenstein (0.7) but still significantly below the augmented baseline. Transitive closure affects the baseline differently in the two datasets. While it adversely affects precision for arXiv, it improves recall for CiteSeer.

Table 3.2 shows the best performance of each of the three algorithms for each dataset. Note that the recall includes blocking, so that the highest recall achievable is 0.993 for CiteSeer and 0.991 for arXiv. LDA-ER outperforms both forms of the baseline for both datasets for all string similarity measures and the improvements are statistically significant. For CiteSeer, LDA-ER gets close to the highest possible recall with very high accuracy. This means that it is able to retrieve almost all duplicates correctly. Improvement over the baseline is greater for arXiv in terms of F1. Also, LDA-ER reduces error rate over the baseline by $22 \%$ for CiteSeer (from $0.9 \%$ to $0.7 \%$ ) and by $20 \%$ for arXiv (from $2.4 \%$ to $1.9 \%$ ). Also, arXiv has more than 64,6000 true duplicate pairs, so that a $1 \%$ improvement in $F 1$ translates to 
Table 3.2: Performance of LDA-ER, $\mathbf{A}$ and $\mathbf{A}^{*}$ for CiteSeer and arXiv datasets. The standard deviation of the $F 1$ is $3 \times 10^{-4}$ for CiteSeer and $1.7 \times 10^{-4}$ for arXiv.

\begin{tabular}{|l|ccc|ccc|}
\hline & \multicolumn{3}{|c|}{ CiteSeer } & \multicolumn{3}{c|}{ arXiv } \\
& P & R & F1 & P & R & F1 \\
\hline \hline A & 0.990 & 0.971 & 0.981 & 0.987 & 0.965 & 0.976 \\
A $^{*}$ & 0.992 & 0.988 & 0.991 & 0.976 & 0.965 & 0.971 \\
LDA-ER & 0.997 & 0.988 & $\mathbf{0 . 9 9 3}$ & 0.991 & 0.971 & $\mathbf{0 . 9 8 1}$ \\
\hline
\end{tabular}

more than 6, 400 correct pairs.

Looking more closely at the resolution decisions from CiteSeer, I was able to identify some interesting combination of decisions by LDA-ER that would be difficult or impossible for an attribute-only model. There are instances in the dataset where reference pairs are very similar but correspond to different author entities. Examples include (liu $j, l u j$ ) and (chang $c$, chiang c). LDA-ER correctly predicts that these are not duplicates. At the same time, there are other pairs that are not any more similar in terms of attributes than the examples above and yet are duplicates. These are also correctly predicted by LDA-ER by leveraging common collaboration patterns. The following are examples: (john $m f$, john $m$ st), (reisbech $c$, reisbeck $c k$ ), (shortliffe e $h$, shortcliffe e h), (tawaratumida s, tawaratsumida sukoya), (elliott g, elliot gl), (mahedevan s, mahadevan sridhar), (livezey b, livezy b), (brajinik g, brajnik g), (kaelbing l p, kaelbling leslie pack), (littmann michael l, littman m), (sondergaard $h$, sndergaard $h$ ) and (dubnick cezary, dubnicki c). An example of a particularly pathological case is (minton s, minton andrew b), which is the result of a parse error. The attribute-only baselines cannot make the right prediction for both these sets of examples simultaneously, whatever the decision 
Table 3.3: LDA-ER Performance over varying number of groups

\begin{tabular}{|c|ccc|ccc|}
\hline & \multicolumn{3}{|c|}{ CiteSeer } & \multicolumn{3}{c|}{ arXiv } \\
\# Grps & P & R & F1 & P & R & F1 \\
\hline \hline 100 & 0.995 & 0.991 & 0.993 & 0.986 & 0.972 & 0.979 \\
200 & $\mathbf{0 . 9 9 7}$ & $\mathbf{0 . 9 8 8}$ & $\mathbf{0 . 9 9 3}$ & 0.988 & 0.972 & 0.980 \\
300 & 0.998 & 0.980 & 0.989 & 0.990 & 0.971 & 0.980 \\
400 & 0.999 & 0.980 & 0.989 & 0.990 & 0.970 & 0.980 \\
500 & & & & $\mathbf{0 . 9 9 1}$ & $\mathbf{0 . 9 7 1}$ & $\mathbf{0 . 9 8 1}$ \\
600 & & & & 0.991 & 0.969 & 0.980 \\
\hline
\end{tabular}

threshold, since they consider names alone.

I was also interested in exploring how the number of collaborative groups affects the performance of the proposed entity resolution algorithm. Table 3.3 records the performance of the group model on the two datasets with varying number of groups. While I observe a general trend where precision improves and recall suffers with more groups, note that the $F 1$ is largely stable over a range of groups.

\subsubsection{Properties of Collaborative Graphs}

While the LDA-ER model shows improvement for both citation datasets, the improvement is much more significant for the arXiv dataset. On investigating why the model shows a larger improvement for arXiv than for CiteSeer, I found some notable differences between the datasets. I call a reference ambiguous if there is more than one author entity with that last name and first initial. There is a significant difference in reference ambiguity between the two datasets - only $0.5 \%$ of the references in CiteSeer are ambiguous while $9 \%$ of arXiv references are ambiguous. A second difference is in the density of the author collaboration graph. The average 


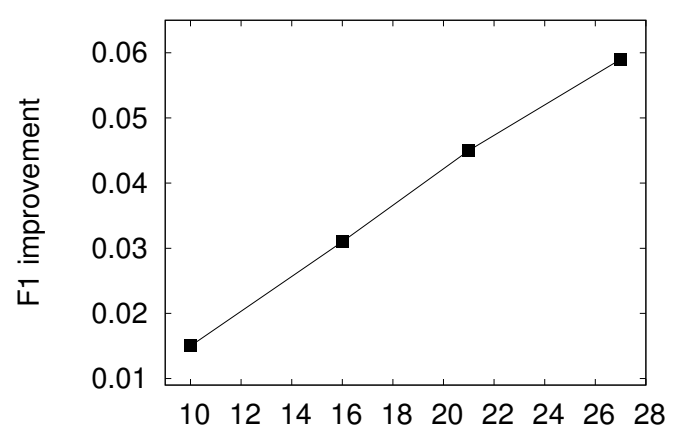

(a) Percentage of ambiguous refs.

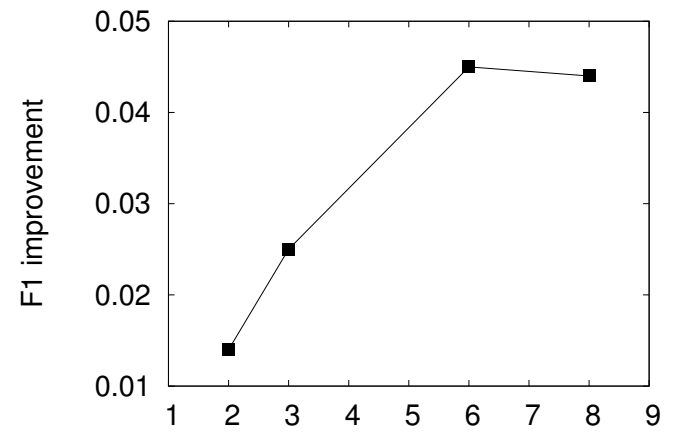

(b) Avg. num. references per author

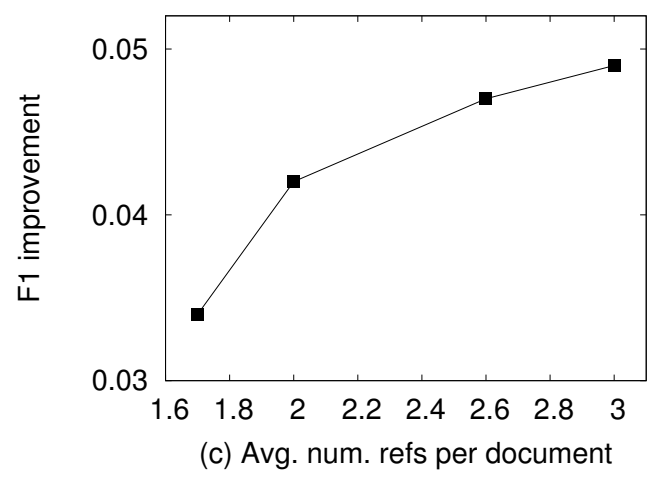

Figure 3.3: Improvement of LDA-ER over $\mathbf{A}^{*}$ for varying (a) ambiguity of references, (b) avg. number of references per author and (c) avg. number of references per document. Other parameters are held constant for each experiment.

number of collaborators per author is 2.15 in CiteSeer and 4.5 in arXiv. Finally, a third significant difference relates to the sample size. While the ratio of the number of references to the number of authors is 2.5 for CiteSeer, for arXiv it is 6.36 . On the other hand, one of the features that is preserved for both datasets is the average number of references per document, which is 1.9 for both.

In order to investigate which of these features is responsible for the performance difference, I ran the algorithm on a range of synthetic datasets generated using the process described in Appendix A. In the setup for experiments with synthetic data, I vary the synthetic dataset parameters one at a time holding the 
others constant. The default values of the parameters are set to reflect the features of the real datasets. The datasets have 1000 authors with an average of 4.5 collaborators. I generate 3000 documents with an average of 2 references per document and $15 \%$ ambiguous references. I explore varying the fraction of ambiguous references, the ratio of references to authors, the average number of collaborators and average number of references per document. Since the results are averaged over different datasets, I present only the improvement in $F 1$ measure observed for the group model over $\mathbf{A}^{*}$.

Figure 3.3 summarizes the trends that we observe. One significant improvement trend is over varying ambiguity in the references. As shown in Figure 3.3(a), it climbs sharply from 0.01 for $10 \%$ ambiguity (as in arXiv) to 0.06 for $27 \%$ reference ambiguity. Figure 3.3(b) shows that LDA-ER naturally benefits from higher sample sizes for the author references. Figure 3.3(c) shows that LDA-ER benefits from a greater number of authors per document. However, no statistically significant trends emerged from the experiments with varying collaboration degree keeping other factors like sample size fixed; some experiments showed larger improvements with higher degree, however the results were not consistent. More thoroughly characterizing properties of the collaborative graph structure that lead to improved entity resolution is an interesting area for future work. 


\subsubsection{Comparison With Collective Relational Clustering}

Before moving on to experiments on synthetically generated data, I briefly look at how the relational clustering approach (CR) that I proposed in Chapter 2 compares with LDA-ER.

Table 3.4: Comparison of LDA-ER with relational clustering(CR)

\begin{tabular}{|l|cc|cc|}
\hline & \multicolumn{2}{|c|}{ CiteSeer } & \multicolumn{2}{c|}{ arXiv } \\
& F1 & secs & F1 & secs \\
\hline \hline CR & 0.995 & 2.7 & 0.985 & 299 \\
LDA-ER & 0.993 & 240 & 0.981 & 36,000 \\
\hline
\end{tabular}

In Table 3.4, I compare the performance and execution times for LDA-ER and CR on CiteSeer and arXiv. I am currently not able to compare them for BioBase, since extending the LDA-ER generative process for multiple attributes is ongoing work. We can see that $\mathbf{C R}$ is superior in terms of performance. However, it requires a similarity threshold to be specified. In comparison, LDA-ER does not require any such threshold and automatically figures out the most likely number of entities. LDA-ER does require the number of groups as a parameter, but we have seen that it is usually easier to specify than the number of entities or a termination threshold. Additionally, LDA-ER automatically discovers hidden group structures among the entities from the observed hyper-edges. The price for this improvement in the case of LDA-ER is significantly longer execution times, as can be seen from Table 3.4. In terms of complexity, LDA-ER runs in $O(i(n t+a))$ time for $n$ references, where $i$ is the number of iterations to converge, $t$ is the number of groups and $a$ is the number of entities discovered. In contrast, CR runs in $O(n k \log n)$ time. In general, the 
inference algorithm for LDA-ER needs to go over many iterations before converging and the hidden constants in the complexity are also high in comparison to $\mathbf{C R}$, as the execution times for CiteSeer and arXiv demonstrate.

So we can see that collective relational entity resolution (CR) and the probabilistic generative model LDA-ER both have their strengths and weaknesses and there is no clear winner in terms of performance. In domains where fast results are needed and a termination threshold can be determined by alternative means, the relational clustering approach may be the preferred choice. In other domains, where a termination threshold is harder to specify, the non-parametric probabilistic model may be more appropriate. Also, the hidden group structure discovered by LDA-ER may be valuable in many domains such as social and collaborative networks.

\subsection{Conclusions}

In this chapter, I have developed a probabilistic generative model for collectively resolving entities in relational data. There is a long history of work on entity resolution. Recently, generative $[67,86]$ and discriminative $[73,98]$ probabilistic approaches have been proposed as well as non-probabilistic algorithms [60, 41]. The proposed model differs from most of the above in that it is unsupervised, does not assume the underlying entities to be known, does not make pairwise decisions and explicitly models relations between entities using group membership. Unlike most existing models, I do not introduce a decision variable for each potential duplicate pair of references, but instead have an entity label for each reference. To model 
collaborative relations between entities, I have introduced a group label for each reference, so that entities coming from the same collaborative group are more likely to be observed in a relation. For author resolution, this means that I model collaborative groups to explain co-authorship relations. The generative process in this model may be viewed as an extension of the Dirichlet Process mixture model: the group labels influence the choice of entities for each author reference in a paper.

Another contribution in my approach is an unsupervised Gibbs sampling algorithm for collective entity resolution. It is unsupervised because I do not make use of a labeled training set and it is collective because the resolution decisions depend on each other through the group labels. Further, the number of entities is not fixed in this model, and I have proposed a novel sampling strategy to estimate the most likely number of entities given the references.

I have demonstrated the utility of the proposed model on two real-world citation datasets. Additionally, I have identified some of the conditions under which these models are expected to provide greater benefit. Areas for future work include extending the models to resolve multiple entity classes, handling multiple attributes of different types and better characterization of collaborative graphs amenable to these models. Though the improved sampling strategy addresses the computational cost of the inference process to some extent, the execution times are orders of magnitude larger than the relational clustering approach. Designing algorithms for faster inference that make this approach scalable to huge datasets is an obvious direction of future research. 


\section{Chapter 4}

\section{Entity Resolution for Queries}

Instead of collectively resolving all the references in a database, in this chapter I investigate collective approaches for resolving entities on the fly for the purpose of answering user queries over a database that has unresolved entity references. The rest of this chapter is organized as follows. In Section 4.1, I motivate the problem of resolving entities for answering queries, and in Section 4.2, I formulate the notion of entity resolution queries. Section 4.3 investigates how the resolution performance of related clusters depends recursively on each other as a result of this algorithm. This motivates a recursive 'expand and resolve' strategy for processing queries. In Section 4.4, I describe and analyze an unconstrained recursive strategy for extracting the references relevant for collectively resolving a query. In Section 4.5, I present my adaptive algorithm that extracts only the 'most informative' references for resolving a given query. I present experimental results on real and synthetic data in Section 4.6 and finally conclude in Section 4.7.

\subsection{Motivativation for Entity Resolution Queries}

In spite of the widespread research interest and the practical nature of the entity resolution problem, many publicly accessible databases remain unresolved, 
or partially resolved, at best. The popular publication databases, CiteSeer and PubMed, are representative examples. CiteSeer contains several records for the same paper or author (going by CiteSeer records, Stuart Russell and Peter Norvig have written more than 100 different books together [86]), while author names in PubMed are not resolved at all. This is due to a variety of reasons, ranging from rapid and often uncontrolled growth of the databases and the computational and other expenses involved. Yet, millions of users access and query such databases everyday, mostly seeking information that, implicitly or explicitly, requires knowledge of the resolved entities. The information gathered from such databases would be significantly more useful or accurate if the entities were resolved.

Motivated by the abundance of such important and unresolved public databases, I formulate the problem of query-time entity resolution. The goal is to enable users to query an unresolved or partially resolved database and resolve the relevant entities on the fly. A user may access several databases everyday and he does not want to clean every database that he queries. He only needs to resolve those entities that matter for his query. For instance, when looking for all books by 'Stuart Russell' in CiteSeer, it is not useful to resolve all other author references in CiteSeer. Also, the resolution needs to be quick, even if it is not entirely accurate.

A possible solution for the query-time entity resolution problem that is both quick and simple is to use attribute-based resolution. However, we have already seen in Chapter 2 and Chapter 3 that collective resolution approaches can significantly improve accuracy over attribute-only baselines. So the goal is to use collective resolution using relationships for queries as well. But this added improvement comes at 
a considerable computation cost arising from the dependencies. This added computational expense makes its application in query-time resolution challenging. Due to its inter-dependent nature, the set of references that influence collective resolution of a query may be very large. In this chapter, I first present a formal analysis of how inter-dependence of different entity resolution decisions affect entity resolution accuracy. This analysis motivates us to design a recursive resolution strategy for query-centric entity resolution. I also present adaptive algorithms for extracting the most relevant references for a query that enable us to resolve entities at query-time, while preserving the gains of collective resolution.

My specific contributions in this chapter are as follows. First, I motivate and formulate the problem of query-time entity resolution. The entity resolution strategy that I use for query-time resolution is the collective relational clustering algorithm (CR) introduced in Subsection 2.3.3. To the best of my knowledge, clustering based on queries in the presence of relations has received little attention. I present a structural analysis of how relational clustering affects entity resolution accuracy and of the convergent nature of resolution performance for a recursive strategy for resolving queries. I also formulate query-time clustering as a resource-constrained problem and propose adaptive strategies for constructing the set of references that influence a query. Finally, I present experimental results on large real-world and synthetic datasets where this strategy enables collective resolution in seconds with minimal loss in accuracy. 


\subsection{Entity Resolution Queries: Formulation}

Let us revisit the four example papers from Section 2.1:

1. W. Wang, C. Chen, A. Ansari, "A mouse immunity model"

2. W. Wang, A. Ansari, "A better mouse immunity model"

3. L. Li, C. Chen, W. Wang, "Measuring protein-bound fluxetine"

4. W. W. Wang, A. Ansari, "Autoimmunity in biliary cirrhosis"

Representing them in the notation introduced for the entity resolution problem in Section 2.2 , we have 10 references $\left\{r_{1}, \ldots, r_{10}\right\}$ in $\mathcal{R}$, where $r_{1}$. Name $=$ 'W Wang', etc. There are 4 hyper-edges $\left\{h_{1}, \ldots, h_{4}\right\}$ in $\mathcal{H}$ for the four papers. According to the ground truth, we have six underlying entities. This is illustrated in Figure 2.1 using a different shading for each entity. For example, the 'Wang's of papers 1, 2 and 4 are the same individual but that from paper 3 is a different person. Also, the 'Chen's from papers 1 and 3 are different individuals. Then, the correct resolution for the example database with 10 references returns 6 entity clusters: $\left\{\left\{r_{1}, r_{4}, r_{9}\right\}\right.$, $\left.\left\{r_{8}\right\},\left\{r_{2}\right\},\left\{r_{7}\right\},\left\{r_{3}, r_{5}, r_{10}\right\},\left\{r_{6}\right\}\right\}$. The first two clusters correspond to 'Wang', the next two to 'Chen', the fifth to 'Ansari' and the last to 'Li'.

Instead of clustering all database references, in many applications, users are interested in just a few of the clusters. For example, we may want to retrieve all papers written by some person named 'W Wang'. I refer to this as an entity resolution query on 'W Wang', since answering it involves knowing the underlying entities. I will assume that queries are specified using $\mathcal{R}$.Name, which is a noisy 
identifier for entities. Since names are ambiguous, treating them as identifiers leads to undesirable results. For example, it would be incorrect to return the set $\left\{r_{1}, r_{4}, r_{8}\right\}$ of all references with name 'W Wang' as the answer to the query. This answer does not indicate that $r_{8}$ is not the same person as the other two. Also, the answer should include the paper by 'W W Wang' $\left(r_{9}\right)$, who is the same entity as the author of the first paper. Therefore, the correct answer to the entity resolution query on 'W Wang' should be the partition $\left\{\left\{r_{1}, r_{4}, r_{9}\right\},\left\{r_{8}\right\}\right\}$.

Different approaches introduces for the general entity resolution problem in Section 2.3 - attribute-based, naive relational and collective relational resolution - can also be used for entity resolution queries. The goal is to use the collective relational entity resolution approach, since it significantly outperforms the other two approaches in terms of entity resolution accuracy. But collective resolution is more expensive computationally and execution time is a key issue for resolving queries. Of the two collective relational entity resolution approaches that I have proposed, collective relational clustering (Section 2.5) is significantly faster than the probabilistic approach, and this is the algorithm that I adapt for resolving queries.

\subsection{Performance Dependencies in Relational Clustering}

The goal is to use the idea of collective resolution for queries. For collective resolution, it is not sufficient to consider only the potential matches for the query in the traditional fashion. For resolving the 'W. Wang' query, the traditional approach simply considers the 4 references from the example that are similar in name - 
the three 'W. Wang' references $\left(r_{1}, r_{4}, r_{8}\right)$ and the 'W. W. Wang' reference $\left(r_{9}\right)$. In contrast, collective resolution benefits by additionally reasoning about the coauthor references, 'Ansari', 'Chen' and 'Li'. As a result, entity resolution accuracy for the query becomes dependent on the resolution accuracy of the related entities. It is necessary to analyze the nature of this dependency in order to understand how collective resolution affects performance for answering queries. In this section, I identify the structural properties of the data that affect collective entity resolution and formally analyze interdependent nature of the resolution performance. This analysis also helps us to understand when relational clustering helps, when it has an adverse effect and how it compares to traditional attribute-based resolution.

Recall that we are given a set of references $\mathcal{R}=\left\{r_{i}\right\}$. The references correspond to an unobserved set of entities $\mathcal{E}$. I assume that each reference $r_{i}$ corresponds to a single entity from $\mathcal{E}$, denoted as $E\left(r_{i}\right)$. An entity resolution algorithm partitions the references into a set of clusters $\mathcal{C}=\left\{c_{i}\right\}$ according to the underlying entities. The accuracy of the resolution depends on how closely the partitioning of the references into clusters corresponds to the underlying entities. I evaluate performance using two different measures. The first measure is recall over each entity $e_{i}$ that measures how many pairs of references that correspond to this entity $e_{i}$ are correctly assigned to the same computed cluster. The second measure is precision for each computed cluster $c_{i}$ measuring how many pairs of references assigned to this cluster $c_{i}$ truly correspond to the same entity. These two measures depend on the attributes of the references and also on the relationships between them, as I describe in the next two subsections. 


\subsubsection{Performance Analysis of Attribute-based Resolution}

First, I analyze how the reference attributes affect entity resolution performance. Each reference $r_{i}$ has a set of attributes. I assume a similarity measure that is defined over the domain of attributes. The traditional attribute-based clustering approach considers only the pair-wise similarity between references based on attribute values for resolving them. Two references $r_{i}, r_{j}$ are said to be $\delta$-similar if their attribute similarity is at least $\delta$. For attribute-based clustering, references are assigned to the same cluster given some similarity threshold $\delta$ if they are $\delta$-similar, or, equivalently, if their attribute similarity is at least $\delta$. In the example, for some similarity measure defined over names and an appropriately determined similarity threshold $\delta$, the three 'W. Wang' references $\left(r_{1}, r_{4}, r_{8}\right)$ would be mapped to one cluster $c_{1}$ and the 'W. W. Wang' reference to a different cluster $c_{2}$. In this case, cluster $c_{1}$ is not fully precise since the pairs $\left(r_{1}, r_{8}\right)$ and $\left(r_{4}, r_{8}\right)$ do not map to the same entity. Also, one of the 'Wang' entities is not completely recalled since the pairs $\left(r_{1}, r_{9}\right)$ and $\left(r_{4}, r_{9}\right)$ are missed.

In order to analyze how attribute-based resolution performs in general given an arbitrary dataset, it is necessary to characterize the dataset in terms of two probabilities defined on the attribute values of the references that it contains. The first probability considers the 'dissimilarity' of attributes for references to the same entity:

- attribute identification probability $\mathbf{a}_{\mathbf{I}}(\delta)$ : the probability that a randomly chosen pair of references from the dataset that correspond to the same entity 
are $\delta$-similar to each other.

The second probability considers the similarity of attributes for references to different entities:

- attribute ambiguity probability $\mathbf{a}_{\mathbf{A}}(\delta)$ : the probability that a randomly chosen pair of references from the dataset that correspond to different entities are $\delta$-similar to each other.

The performance of the attribute-based clustering algorithm can be evaluated using these two probabilities. Given a particular $\delta$, the references pairs that are correctly recalled for any domain entity $e$ are those that are $\delta$-similar. This is exactly what $a_{I}(\delta)$ denotes. Therefore the average recall for $e$ is given by $R(e, \delta)=a_{I}(\delta)$. On the other hand, the references that adversely affect precision for a particular $\delta$ are those that are $\delta$-similar to references to other entities. Therefore the average precision for any computed cluster $c$ is $P(c, \delta)=1-a_{A}(\delta)$. Alternatively, the imprecision is given by $I(c, \delta) \equiv 1-P(c, \delta)=a_{A}(\delta)$.

\subsubsection{Characterizing Relations}

In addition to attributes, many domains have additional relational structure, which I make use of for collective entity resolution. I now analyze how such relationships affect entity resolution accuracy. As before, I use the relational structure in the data as a set of co-occurrence relations $\mathcal{H}=\left\{h_{j}\right\}$ over the references. In general, references to any entity $e_{i}$ may co-occur frequently with references to a set of other entities $\left\{e_{i 1}, \ldots, e_{i k}\right\}$. These entities are called the neighbors $N\left(e_{i}\right)$ of entity $e_{i}$. 

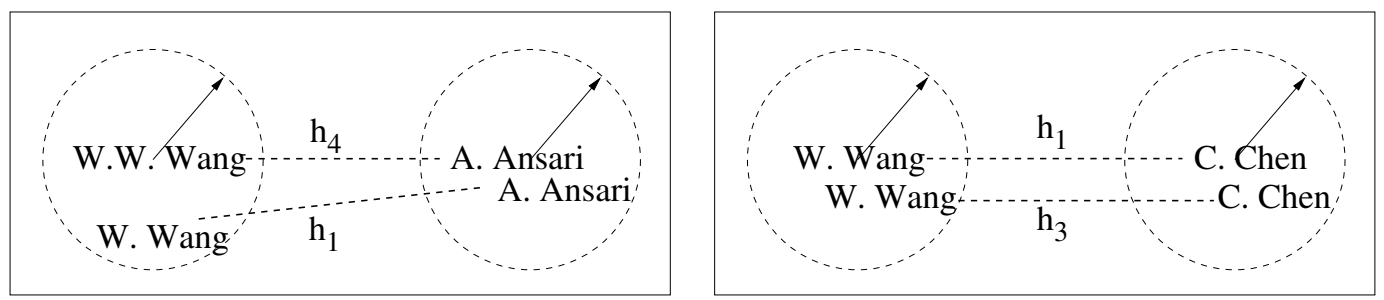

Figure 4.1: Illustration of (a) identifying relation and (b) ambiguous relation using references from the running example. Dashed lines represent co-occurrence relations and dashed circles show $\delta$-boundaries around references.

I use the co-occurrence relations among the references for collective entity resolution. Any pair of references that are $\delta$-similar are candidates for clustering. However, for collective resolution, they are not clustered together blindly. Only those pairs that at least $\epsilon$-similar (where $\epsilon>\delta$ ) are clustered based on their attributes alone. For others that are in the region of uncertainty between $\delta$ and $\epsilon$, relational evidence is taken into account. Specifically, such a pair $\left(r_{i}, r_{i}^{\prime}\right)$ is clustered together only if they co-occur with references $r_{j}$ and $r_{j}^{\prime}$ respectively, and $\left(r_{j}, r_{j}^{\prime}\right)$ is already clustered together.

To analyze the impact of co-occurrence relations on collective entity resolution accuracy, I focus on two structural scenarios for the underlying domain. Consider again two references $r_{i}$ and $r_{i}^{\prime}$ that are $\delta$-similar. Reference $r_{i}$ co-occurs with $r_{j}$ through relation $h$, and $r_{i}^{\prime}$ co-occurs with $r_{j}^{\prime}$ through relation $h^{\prime}$ such that $r_{j}$ and $r_{j}^{\prime}$ are also $\delta$-similar. Thus both pairs $\left(r_{i}, r_{i}^{\prime}\right)$ and $\left(r_{j}, r_{j}^{\prime}\right)$ are candidates for clustering. Without loss of generality, let us assume that the $\left(r_{j}, r_{j}^{\prime}\right)$ pair get clustered together first by the relational clustering algorithm. Then the other pair also gets clustered at some later stage by considering this relational evidence. To see if this is accurate, I consider two situations. The first is shown in Figure 4.1(a) where both pairs truly 
correspond to the same entity. Then the collective resolution decision is correct and $h$ is an identifying relationship for reference $r_{i}$. But now consider the second scenario where $\left(r_{j}, r_{j}^{\prime}\right)$ do not correspond to the same entity, and neither does the other pair $\left(r_{i}, r_{i}^{\prime}\right)$. This is illustrated in Figure 4.1(b). Then the decision to resolve $\left(r_{j}, r_{j}^{\prime}\right)$ as coreferent is incorrect and the co-occurrence relation consequently leads to the incorrect resolution of $\left(r_{i}, r_{i}^{\prime}\right)$ as well. This is the scenario when collective resolution hurts accuracy, and $h$ is an ambiguous relationship for $r_{i}$.

In general, a reference $r_{i}$ can have a co-occurrence relation $h$ that connects multiple other references. Each of these other references in $h$ can influence $r_{i}$ to be resolved correctly or incorrectly. When co-occurrence relations connect more than two references, the way the relational evidence is aggregated depends on the specific relational similarity measure that is employed. Here, $h$ an ambiguous relationship for $r_{i}$ if the majority of the other references in $h$ can lead to $r_{i}$ being incorrectly clustered. Otherwise, $h$ is called an identifying relationship for $r_{i}$. Specifically, I define:

- identifying relationship probability $\mathbf{r}_{\mathbf{I}}(\delta)$ : the probability that a randomly chosen reference from the dataset has an identifying relationship

- ambiguous relationship probability $\mathbf{r}_{\mathbf{A}}(\delta)$ : the probability that a randomly chosen reference from the dataset has an ambiguous relationship.

Having defined these two probabilities, I now analyze the performance of the relational clustering algorithm. Let us first consider recall. Observe that the recall for any entity $e$ depends recursively on the recall of its neighbor entities $N(e)$. A pair 
of references for entity $e$ is recalled correctly on the basis of attribute alone with probability $a_{I}$ (the identifying attribute probability). Additionally, co-occurrence relationships connect references to entity $e$ with references to other entities from $N(e)$. If a reference pair from $e$ is not recalled on the basis of attributes, it may still be recalled correctly when one of them has an identifying relationship with a neighbor entity, and that neighbor is recalled correctly. Denoting as $R(e)$ the recall for $e$ and that of its neighbors as $R(N(e))$, we have:

$$
R(e)=a_{I}(\epsilon)+\left(1-a_{I}(\epsilon)\right) \times r_{I}(\delta) \times R(N(e))
$$

On the other hand, a computed cluster $c$ is imprecise on the basis of its attributes alone with probability $a_{A}$. In Subsection 2.3.3, I defined the neighbors for any cluster as all the other clusters with which it shares co-occurrence relations. Aside from the effect of attributes, a cluster can also imprecise when references have ambiguous relationships and the neighbor clusters are imprecise. Formally, the imprecision (1-precision) $I(c)$ for any computed cluster $c$ turns out to be:

$$
I(c)=a_{A}(\epsilon)+\left(1-a_{A}(\epsilon)\right) \times r_{A}(\delta) \times I(N(c))
$$

Recall that any entity e can have multiple neighbors $\left\{e_{i}^{n}\right\}$, and similarly, any entity computed cluster $c$ can have multiple neighbor clusters $\left\{c_{i}^{n}\right\}$. To completely analyze the dependence on neighbors, assume that references to entity $e$ co-occur with references to $e_{i}^{n}$ with probability $p_{i}^{n}$. Also, $f_{i}^{n}$ denotes the fraction of cooccurrence relations for cluster $c$ that are shared with neighbor $c_{i}^{n}$. Then recall and 
imprecision are given as:

$$
\begin{aligned}
& R(e)=a_{I}(\epsilon)+\left(1-a_{I}(\epsilon)\right) \times r_{I}(\delta) \times \sum_{i=1}^{k} p_{i}^{n} R\left(e_{i}^{n}\right) \\
& I(c)=a_{A}(\epsilon)+\left(1-a_{A}(\epsilon)\right) \times r_{A}(\delta) \times \sum_{i=1}^{k} f_{i}^{n} I\left(c^{i}\right)
\end{aligned}
$$

The above equations enable us to analyze the advantages and disadvantages of relational clustering for entity resolution. Once the similarity thresholds $\epsilon$ and $\delta$ have been fixed, Eq. (4.3) shows that relational clustering increases recall beyond that achievable using attributes alone. This improvement is greater when the probability of identifying relationships is higher. On the flip side, imprecision also increases with relational clustering as shown by Eq. (4.4). Normally, a low attribute threshold $\epsilon$ that corresponds to high precision is used and then recall is increased using relational clustering. When the probability of ambiguous relations $r_{A}$ is small, the accompanying increase in imprecision is negligible and performance is improved overall. However, as $r_{A}$ increases, relational clustering becomes less effective. Thus the balance between ambiguous and identifying relations determines the benefit of relational clustering. When $r_{A}$ is high compared to $r_{I}$, imprecision increases faster than recall and performance is adversely affected by the use of relational clustering compared to attribute-based clustering.

\subsection{Two-Stage Query Processing}

I now focus on a strategy for applying collective resolution for query processing and extend the analysis from Section 4.3 to understand how it improves resolution accuracy over attribute based approaches. For entity resolution queries, we are given 
a query name or query reference $r_{q}$ and the goal is to retrieve all references in the dataset that belong to the same entity $e_{q}$ as $r_{q}$, i.e., $e_{q}=E\left(r_{q}\right)$. One possibility is to extend the traditional attribute-based entity resolution approach for resolving queries as well — for a specific similarity threshold $\delta$, all references that are $\delta$ similar to the query reference would be retrieved as cluster $c_{q}$. We have seen that the accuracy for this simple algorithm is given by $R\left(e_{q}\right)=a_{I}$ and $I\left(c_{q}\right)=a_{A}$, where $a_{I}$ and $a_{A}$ are the two attribute probabilities for the domain. The problem of interest is resolving queries using co-occurrence information. But it is clearly not desirable to cluster all database references collectively, as is done in the normal collective clustering setting. Instead, it is necessary to localize collective relational clustering, where we the neighboring clusters are recursively identified in order to find the references for $c_{q}$. In this section, I propose a two-phase query processing strategy consisting of an extraction phase followed by a resolution phase. In the extraction phase, the goal is to extract the relevant set of references $\operatorname{Rel}\left(r_{q}\right)$ for answering the query accurately and then, in the resolution phase, I perform collective resolution on $\operatorname{Rel}\left(r_{q}\right)$. I also use structural analysis to analyze the performance of localized collective clustering for query resolution.

Recall that for relational clustering using co-occurrence relations, all references that are $\delta$-similar are not blindly clustered together. Instead, the co-occurrence relations are used to consider the clusters of the co-occurring references. To apply the same approach for localized collective clustering, I introduce two expansion operators for constructing the relevant set for an entity resolution query $r_{q}$ by including all references that are needed for answering the query collectively. 


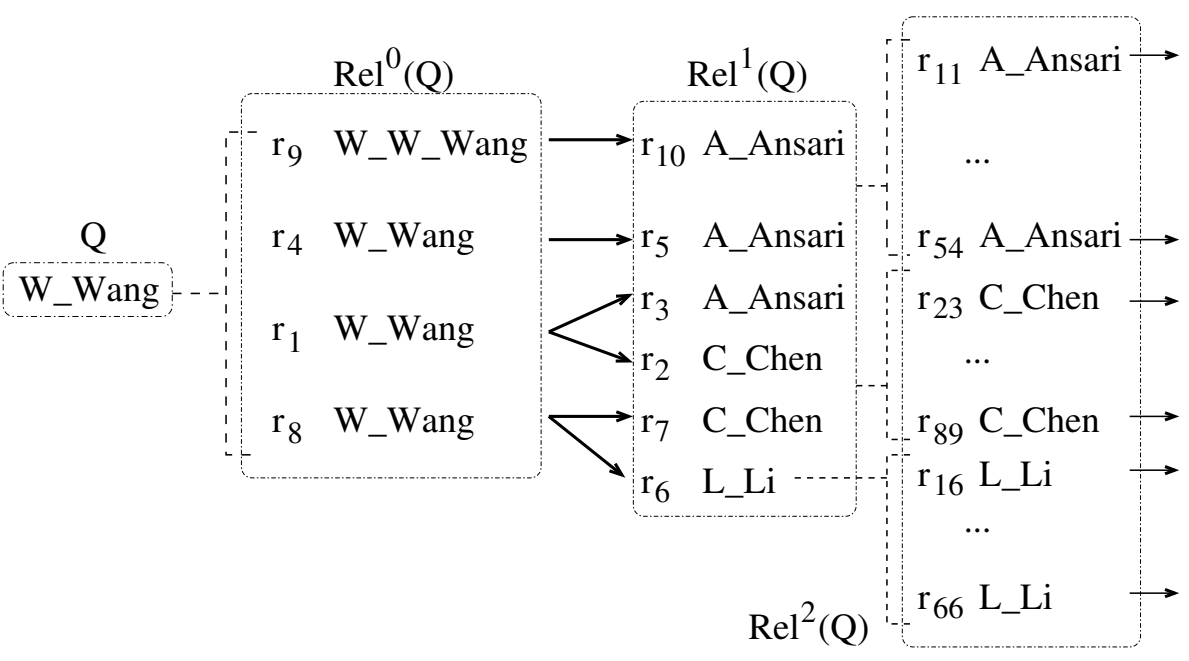

Figure 4.2: Relevant set for query 'W. Wang' using h-expansion and a-expansion alternately

First, I denote as the level-0 references all references that are $\delta$-similar in terms of attributes to the query and are potential cluster members for $c_{q}$. The first operator is the attribute expansion operator $X_{A}$ or a-expansion for short. For an reference $r, X_{A}(r)$ returns all references whose attributes exactly match that of $r$ or are $\delta$-similar to it. For a query $r_{q}$, the level-0 references can be retrieved by expanding $r_{q}$ as $\operatorname{Re}^{0}\left(r_{q}\right)=X_{A}\left(r_{q}\right)$. The first step in Figure 4.2 shows n-expansion on 'W Wang' in the example.

To consider co-occurrence relations, I construct the level-1 relevant references by including all references that co-occur with level-0 references. For this, I use the second operator, which is hyper-edge expansion $X_{H}$, or h-expansion. For any reference $r, X_{H}(r)$ returns all references that share a hyper-edge with it. Collective entity resolution, it is considers all related references for each reference. Therefore, it is necessary to perform h-expansion on the references at level-2 $\left(\operatorname{Rel}^{0}\left(r_{q}\right)\right)$ to construct the level-1 references: $\operatorname{Rel}^{1}\left(r_{q}\right)=X_{H}\left(\operatorname{Rel}^{0}\left(r_{q}\right)\right)$. Figure 4.2 illustrates 
this operation in the example.

To perform collective clustering for the query, the references at level-1 need to be clustered as well. One option for level-1 references is attribute-based clustering using a conservative $\epsilon$-similarity to keep imprecision to a minimum. The analysis technique from before is again useful for evaluating the performance for this approach. Expanding from Eq. (4.3), and using $a_{I}(\epsilon)$ for the recall of each neighboring entity for $e_{q}$, the recall for the query entity is:

$$
R\left(e_{q}\right)=a_{I}(\epsilon)+\left(1-a_{I}(\epsilon)\right) \times r_{I}(\delta) \times \sum_{i=1}^{k} p_{i}^{n} a_{I}(\epsilon)
$$

Observing that $\sum_{i=1}^{k} p_{i}^{n}=1$ allows us to simplify the above expression for recall:

$$
\begin{aligned}
R\left(e_{q}\right) & =a_{I}(\epsilon)+\left(1-a_{I}(\epsilon)\right) \times r_{I}(\delta) \times a_{I}(\epsilon) \\
& =a_{I}(\epsilon)\left[1+\left(1-a_{I}(\epsilon)\right) r_{I}(\delta)\right]
\end{aligned}
$$

Similarly, the imprecision for the computed cluster $c_{q}$ can be expressed as

$$
I\left(c_{q}\right)=a_{A}(\epsilon)\left[1+\left(1-a_{A}(\epsilon)\right) r_{A}(\delta)\right]
$$

So we can see that attribute-clustering of the the first level neighbors potentially increases recall for the query entity $e_{q}$, but imprecision goes up as well. However, when the balance between $r_{A}$ and $r_{I}$ is favorable, the increase in imprecision will be insignificant and much smaller than the corresponding increase in recall, so that there is an overall performance improvement.

Is it possible to do better than this? We can go a step further and consider cooccurrence relations for clustering the level-1 references as well. So, instead of considering $\epsilon$-similarity for references in level-1 as before, I find all of their $\delta$-similar ref- 
erences, which I call level-2 $\left(\operatorname{Rel}^{2}\left(r_{q}\right)\right)$, using a-expansion: $\operatorname{Rel}^{2}\left(r_{q}\right)=X_{A}\left(\operatorname{Rel}^{1}\left(r_{q}\right)\right)$. Then, to retrieve the second order neighbors, I consider level-3 references - those that co-occur with level-2 references - using h-expansion on the em level-2 references: $\operatorname{Rel}^{3}\left(r_{q}\right)=X_{H}\left(\operatorname{Rel}^{2}\left(r_{q}\right)\right)$. Then, as before, I cluster the level-3 references by simply using $\epsilon$-similarity of their attributes. In order to see how this affects accuracy for the query, a few algebraic steps yield the following:

$$
\begin{aligned}
R\left(e_{q}\right) & =a_{I}\left[1+\left(1-a_{I}\right) r_{I}+\left(1-a_{I}\right)^{2} r_{I}^{2}\right] \\
I\left(c_{q}\right) & =a_{A}\left[1+\left(1-a_{A}\right) r_{A}+\left(1-a_{A}\right)^{2} r_{A}^{2}\right]
\end{aligned}
$$

This recursive growth of the relevant set can be continued further. Formally, for a query $r_{q}$, the expansion process alternates between a-expansion and h-expansion:

$$
\begin{aligned}
& \operatorname{Rel}^{i}\left(r_{q}\right)=X_{A}\left(r_{q}\right) \quad \text { for } i=0 \\
& X_{H}\left(\operatorname{Re}^{i-1}\left(r_{q}\right)\right) \quad \text { for odd } i \\
& X_{A}\left(\operatorname{Rel}^{i-1}\left(r_{q}\right)\right) \quad \text { for even } i
\end{aligned}
$$

As I proceed recursively and consider higher order co-occurrences for localized clustering, additional terms appear in the expressions for precision and recall, so that for $n^{\text {th }}$-order co-occurrences I get a geometric progression with $n+1$ terms for both. But this does not imply that this process needs to be continued to arbitrary levels to get optimum benefit. The common ratio for the two geometric progressions are $\left(1-a_{I}\right) r_{I}$ and $\left(1-a_{A}\right) r_{A}$ respectively. Both of these are significantly smaller than 1 and therefore the progressions converge very quickly with increasing co-occurrence level. So the improvement in resolution accuracy for the query $r_{q}$ falls off quickly 
with expansion depth, and the expansion process can be terminated at some cut-off $\operatorname{depth} d^{*}$ :

$$
\operatorname{Rel}\left(r_{q}\right)=\bigcup_{i=0}^{d^{*}} \operatorname{Rel}^{i}(Q)
$$

Also, the size of the relevant set can be significantly reduced by restricting attribute expansion beyond level-0 to exact a-expansion $X_{A}^{e}(r)$ that only considers references with exactly the same attribute as $r$. Interestingly, it can be shown that the restricted strategy that alternates between exact a-expansion and h-expansion does not affect recall significantly.

\subsection{Adaptive Query Expansion}

The query expansion strategy from the previous section is unconstrained in that it blindly expands all references in the current relevant set and also includes all new references generated by an expansion operation. However, for many domains the size of the relevant set resulting from such unconstrained expansion is prohibitive for query-time resolution even for small expansion depths. Given the limited time to process a query, one solution is to include the references that are most helpful for resolving the query. To illustrate using the example from Figure 4.2, observe that 'Chen' and 'Li' are significantly more common or 'ambiguous' names than 'Ansari' — even different 'W. Wang' entities are likely to have collaborators named 'Chen' or 'Li'. Therefore, when h-expanding $\operatorname{Rel}^{0}\left(r_{q}\right)$ for 'W. Wang', 'Ansari' is more informative than 'Chen' or 'Li'. Similarly, when n-expanding $\operatorname{Rel}^{1}\left(r_{q}\right)$, we can choose not to expand the name 'A. Ansari' any further, since two 'A. Ansari' references are 
very likely to be coreferent. But more evidence is needed for the 'Chen's and 'Li's. To describe this formally, the ambiguity of a name $n$ is the probability that any two references $r_{i}$ and $r_{j}$ in the database that have this name $\left(r_{i}\right.$. Name $=r_{j}$. Name $\left.=n\right)$ are not coreferent: $A m b(n)=P\left(E\left(r_{i}\right) \neq E\left(r_{j}\right)\right)$. The goal of adaptive expansion is to add less ambiguous references to the relevant set and, of the references currently in the relevant set, expand the most ambiguous ones.

For adaptive hyper-edge expansion, upper-bound $h_{\max }$ is set on the number of new references that h-expansion at a particular level can generate. This can be represented formally as $\left|X_{H}\left(\operatorname{Rel}^{i}\left(r_{q}\right)\right)\right| \leq h_{\max }\left|\operatorname{Rel}^{i}\left(r_{q}\right)\right|$. The value of $h_{\max }$ may depend on depth $i$ but it is small enough to rule out full h-expansion of the current relevant set. Then, given $h_{\max }$, the strategy is to choose the least ambiguous references from $X_{H}\left(\operatorname{Rel}^{i}\left(r_{q}\right)\right)$, since they provide the most informative evidence for resolving the references in $\operatorname{Rel}^{i}\left(r_{q}\right)$. I sort the h-expanded references in increasing order of ambiguity and select the first $k$ from them, where $k=h_{\max }\left|\operatorname{Rel}^{i}\left(r_{q}\right)\right|$.

$$
\operatorname{Re}_{\text {adapt }}^{i}\left(r_{q}, h_{\text {max }}\right)=\text { Least } A m b\left(k, X_{H}\left(\operatorname{Rel}_{\text {adapt }}^{i-1}\left(r_{q}\right)\right)\right)
$$

The setting for adaptive attribute expansion is very similar. For some positive number $a_{\text {max }}$, exact a-expansion of $\operatorname{Re}^{i}\left(r_{q}\right)$ is allowed to include at most $a_{\text {max }}\left|\operatorname{Rel}^{i}\left(r_{q}\right)\right|$ references. Note that now the selection preference needs to be flipped — more ambiguous names need more evidence, so they are expanded first. So I can sort $X_{A}^{e}\left(\operatorname{Rel}^{i}\left(r_{q}\right)\right)$ in decreasing order of ambiguity and select the first $k$ from the sorted list, where $k=a_{\text {max }}\left|\operatorname{Rel}^{i}\left(r_{q}\right)\right|$. But this could potentially retrieve only references for the most ambiguous name, totally ignoring references with any other 
name. To avoid this, I choose the top $k$ ambiguous references from $\operatorname{Rel}^{i}\left(r_{q}\right)$ before expansion, and then expand the references so chosen.

$$
\operatorname{Rel}_{\text {adapt }}^{i}\left(r_{q}, n_{\text {max }}\right)=X_{A}^{e}\left(\operatorname{Most} A m b\left(k, \operatorname{Rel}_{\text {adapt }}^{i}\left(r_{q}\right)\right)\right)
$$

Though this cannot directly control the number of new references added, $\mu_{r} \times k$ is a reasonable estimate, where $\mu_{r}$ is the average number of references per name.

The adaptive expansion scheme proposed in this section is crucially dependent on the estimates of name ambiguity. For estimating ambiguity, I make use of the strategy described in Subsection 2.4.4, where I estimate the ambiguity of one attribute using a second attribute. This scheme worked quite well for us, as I illustrate in the next section.

\subsection{Experimental Results}

For experimental evaluation of the query-time resolution strategies, I resorted to both real and synthetically generated datasets. First, I describe the experiments performed on real datasets and then I move on to experiments on synthetic data.

\subsubsection{Experiments on Real Data}

For real data, I used two citation datasets that I have already described in Section 2.6. The first dataset, arXiv, contains papers from high energy physics and was used in KDD Cup 2003 ${ }^{1}$. It has 58,515 references to 9,200 authors, contained

\footnotetext{
${ }^{1}$ http://www.cs.cornell.edu/projects/kddcup/index.html
} 

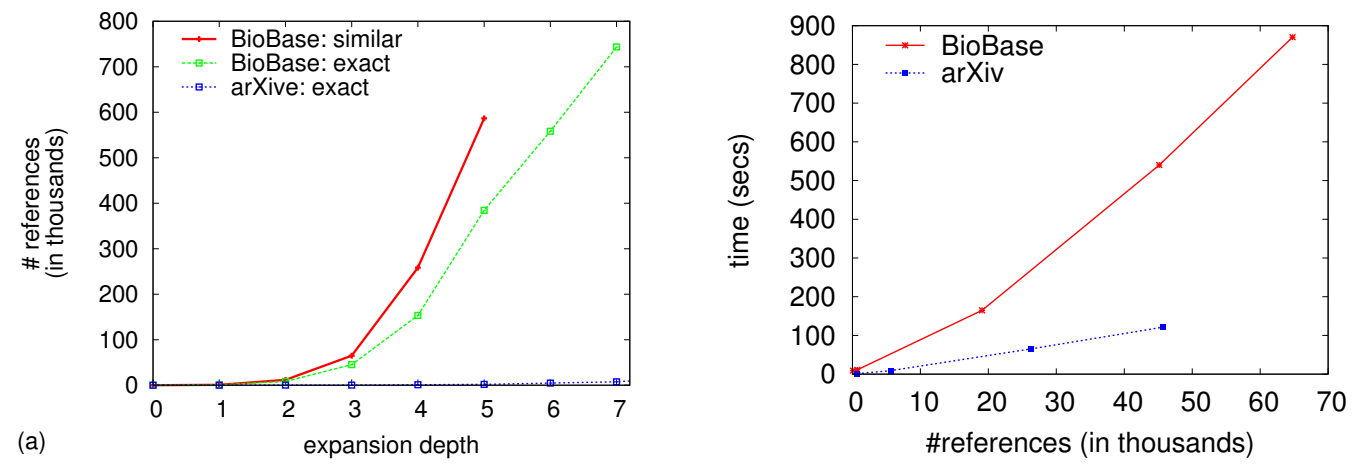

Figure 4.3: (a) Size of the relevant set for increasing expansion depth for sample queries in arXiv and BioBase (b) Execution time of RC-ER with increasing number of references

in 29,555 publications. The second dataset is the Elsevier BioBase database ${ }^{2}$ of publications from biology. It includes all publications under 'Immunology and Infectious Diseases' between years 1998 and 2001. This dataset contains 156,156 publications with 831,991 author references. Unlike arXiv, BioBase includes keywords, topic classification, language, country of correspondence and affiliation of the corresponding author as attributes of the each paper, which I use as attributes for resolution in addition to author names.

For entity resolution queries in arXiv, I selected all ambiguous names that correspond to more than one author entity. This gave us 75 queries with the number of true entities for each varying from 2 to 11 (average 2.4). For BioBase, I query the top 100 author names with the highest number of references. The average number of references for each of these 100 names is 106. The number of entities for each name ranges from 1 to 100 (average 32), thereby providing a wide variety of entity resolution settings over the queries.

\footnotetext{
${ }^{2}$ http://help.sciencedirect.com/robo/projects/sdhelp/about_biobase.htm
} 
I begin by exploring the growth rate of the relevant set for a query over expansion depth in the two datasets. Figure 4.3(a) plots the size of the relevant set for a sample query on the name 'T. Lee' for arXiv and 'M. Yamashita' for BioBase. The growth rate for the arXiv query is moderate. The number of references with name 'T. Lee' is 7 , which is the number of relevant references at depth 0 , and the size grows to 7,500 at depth 7 . In contrast, for BioBase the plots clearly demonstrate the exponential growth of the relevant references with depth for both name expansion strategies. There are 84 relevant references at depth 0 . When references are expanded using name similarity expansion, there are 722 relevant references at depth 1, 65,000 at depth 3 and more than 586,000 at depth 5. This is for a very restricted similarity measure where two names are considered similar only if their first initials match and the last names have the same first character and differ by at most 2 characters. A more liberal measure would result in a significantly faster growth. I also observe that for exact expansion, the growth is slower but I still have 45,000 references at depth 3,384,000 at depth 5 and 783,000 by depth 7 . The growth rates for these two examples from arXiv and BioBase are typical for all of the queries in these two datasets.

Next, in Figure 4.3(b), we observe how the relational clustering algorithm CR scales with number of references. All execution times are reported on a Dell Precision 870 server with $3.2 \mathrm{GHz}$ Intel Xeon processor and $3 \mathrm{~GB}$ of memory. The plot shows that the algorithm scales linearly with increasing references, but the gradient is different for the two datasets mainly due to the difference in the average number of references per hyperlink. This suggests that $\mathbf{C R}$ is well-suited for query- 
time resolution for arXiv. But for BioBase, it would require up to 600 secs for 40,000 references and up to 900 secs for 65,000 . So it is not possible to use $\mathbf{C R}$ for query-time resolution in BioBase even for depth 3 with unconstrained expansion.

In my next experiment, I evaluate several algorithms for entity resolution queries. I compare entity resolution accuracy of the pair-wise co-reference decisions using the F1 measure (which is the harmonic mean of precision and recall). For a fair comparison, I consider the best F1 for each of these algorithms over all possible thresholds for determining duplicates. For the algorithms, I compare attribute-based entity resolution (A), naive relational entity resolution that uses attributes of related references (NR), and the relational clustering algorithm (CR) for collective entity resolution using unconstrained expansion up to depth 3 . I also consider transitive closures over the pair-wise decisions for the first two approaches ( $\mathbf{A}^{*}$ and $\left.\mathbf{N R}^{*}\right)$. For attribute similarity, I use the Soft TF-IDF with Jaro-Winkler similarity for names, which has been shown to perform the best for name-based resolution [16], and TF-IDF similarity for the other textual attributes.

The average F1 scores over all queries are plotted in Table 4.1 for each algorithm in the two datasets. It shows that $\mathbf{C R}$ improves accuracy significantly over the baselines. For example in BioBase, the improvement is $21 \%$ over A and NR, $25 \%$ over $\mathbf{A}^{*}$ and $13 \%$ over $\mathbf{N R}^{*}$. This validates the potential benefits of collective resolution, as shown by recent research $[98,41,73]$ in the context of offline cleaning, and motivates its application for query-time entity resolution. Significantly, most of the accuracy improvement comes from the depth-1 relevant references. For 56 out of the 100 BioBase queries accuracy does not improve beyond the depth-1 relevant 
Table 4.1: Entity resolution accuracy (F1) for different algorithms over 75 arXiv queries and 100 BioBase queries

\begin{tabular}{|l|c|c|}
\hline & arXiv & BioBase \\
\hline \hline A & 0.721 & 0.701 \\
A $^{*}$ & 0.778 & 0.687 \\
NR & 0.956 & 0.710 \\
NR* & 0.952 & 0.753 \\
CR Depth-1 & 0.964 & 0.813 \\
CR Depth-3 & 0.970 & 0.820 \\
\hline
\end{tabular}

references and for the remaining the average improvement is $2 \%$. However, for 8 of the most ambiguous queries, accuracy improves by more than 5\%, the biggest improvement being as high as $27 \%$ (from 0.67 to 0.85 F1). Such instances are fewer for arXiv, but the biggest improvement is $37.5 \%$ (from 0.727 to 1.0 ). This confirms that while there are potential benefits to looking at greater depths, the benefits fall off quite quickly on average beyond depth 1.

The first set of experiments show the benefits of CR. Next, I measure the processing times over unconstrained relevant sets up to depth 3 for all queries in the two datasets. For arXiv, the average processing time of 1.6 secs (with 406 references in the relevant set on average) is quite acceptable. However, it is more than 10 minutes for BioBase (avg. relevant set size is 44,129), which clearly necessitates adaptive strategies for relevant set construction.

Next, I investigate the effectiveness of the adaptive expansion strategy on BioBase. For estimating ambiguity of references, I use last names with first initial as the secondary attribute. This resulted in very good estimates of ambiguity — the ambiguity estimate for a name is strongly correlated (correlation coeff. 0.8) with the 
number of entities for that name. First, I perform constrained resource experiments for h-expansion. For each query $r_{q}$, I construct the relevant set $\operatorname{Rel}\left(r_{q}\right)$ with cutoff depth $d^{*}=1$ and use adaptive h-expansion at depth 1 . The resource constraint parameter $h_{\max }$ is set to 4 . I compare three different adaptive h-expansion strategies: choosing (a) the least ambiguous references, (b) the most ambiguous references and (c) randomly. Then, for each query, I evaluate entity resolution accuracy using $\mathbf{C R}$ on the adaptive relevant sets constructed using these three strategies. The average accuracies for the three strategies over all 100 queries are shown in the first column of Table 4.2. Least ambiguous selection, which is the strategy that I propose, clearly shows the biggest improvement and most ambiguous the smallest, while random selection is in between. Notably, even without many of the depth-1 references, all of them improve accuracy over $\mathbf{N R}^{*}$ with collective resolution.

I perform a similar set of experiments for evaluating adaptive attribute expansion. For each query, I construct the relevant set $\operatorname{Rel}\left(r_{q}\right)$ with $d^{*}=3$ using adaptive a-expansion at depth 1 and unconstrained collaborator expansion at depths 1 and 3. The resource constraint parameter $a_{\max }$ is set to 0.2 , so that on average 1 out of 5 names are expanded. Again, I compare three strategies: expanding (a) the least ambiguous names, (b) the most ambiguous names and (c) random names. The average accuracies for the three schemes over all 100 queries are listed in the second column of Table 4.2. The experiment with collaborator expansion does not bring out the difference between the three schemes as clearly. This is because I am comparing a-expansion at depth 3 and, on average, not much improvement can be obtained beyond depth 1 anyway. But it shows that almost all the benefit at depth 3 comes 
Table 4.2: Resolution accuracy in F1 with different adaptive expansion strategies

\begin{tabular}{|l|cc|}
\hline & h-expansion & a-expansion \\
\hline \hline Least Ambiguous & 0.790 & 0.815 \\
Most Ambiguous & 0.761 & 0.821 \\
Random & 0.770 & 0.820 \\
\hline
\end{tabular}

from the proposed strategy of expanding the most ambiguous names.

The above two experiments demonstrate the effectiveness of the two adaptive expansion schemes in isolation. Now, I present the results when I use them together. For each of the 100 queries, I construct the relevant set $\operatorname{Rel}\left(r_{q}\right)$ with $d^{*}=3$ using adaptive h-expansion and adaptive exact n-expansion. Since most of the improvement from collective resolution comes from depth-1 references, I consider two different experiments. In the first, I use adaptive expansion only at depths 2 and beyond (AX-2) and unconstrained h-expansion at depth 1. In the second(AX-1), I use adaptive h-expansion even at depth 1 , with $h_{\max }=6$. For both of them, I use adaptive expansion at higher depths 2 and 3 with parameters $h_{\max }=3$ at 3 and $a_{\max }=0.2$ at 2.

In Table 4.3, I compare the two adaptive schemes against unconstrained expansion with $d^{*}=3$ over all queries. Clearly, accuracy remains almost unaffected for both schemes. First, note that AX-2 matches the accuracy of unconstrained expansion and shows almost the same improvement over depth 1 even though it a-expands a small fraction of $\operatorname{Rel}^{1}(Q)$ — the average size of the relevant set reduces to 5,500 from 44,000. More significantly, AX-1 also matches this improvement even without including many depth-1 references. This reduction in the size of the relevant 
Table 4.3: Comparison between unconstrained and adaptive expansion for BioBase

\begin{tabular}{|l|ccc|}
\hline & Unconstrained & AX-2 & AX-1 \\
\hline \hline relevant-set size & $44,129.5$ & $5,510.52$ & $3,743.52$ \\
time (cpu secs) & 606.98 & 43.44 & 31.28 \\
accuracy (F1) & 0.821 & 0.818 & 0.820 \\
\hline
\end{tabular}

set has an immense impact on the query processing time. The average processing time drops from more than 600 secs for unconstrained expansion to 43 secs for AX-2 and further to just 31 secs for $\mathbf{A X}-\mathbf{1}$, thus making it possible to use collective entity resolution for query-time resolution.

As a further improvement, I investigate if processing time can be reduced by setting expansion depth $d^{*}$ adaptively, depending on the ambiguity of the query name, as compared to a fixed $d^{*}$ for all queries. In a simple setup, I set $d^{*}$ to 1 for queries where the number of different first initials for a last name is less than 10 (out of 26), and explore depth 2 only for more ambiguous queries. This reduced expansion depth from 2 to 1 for 18 out of the 100 queries. As a result, the average processing time for these queries was reduced by $35 \%$ to 11.5 secs from 17.7 secs with no reduction in accuracy. For three of these queries, original processing time at depth 2 was greater than 30 secs. In these preliminary experiments, I only evaluated the original set of 100 queries that are inherently ambiguous. In a more general setting, where a bigger fraction of queries have lower ambiguity, the impact is expected to be even more significant. 

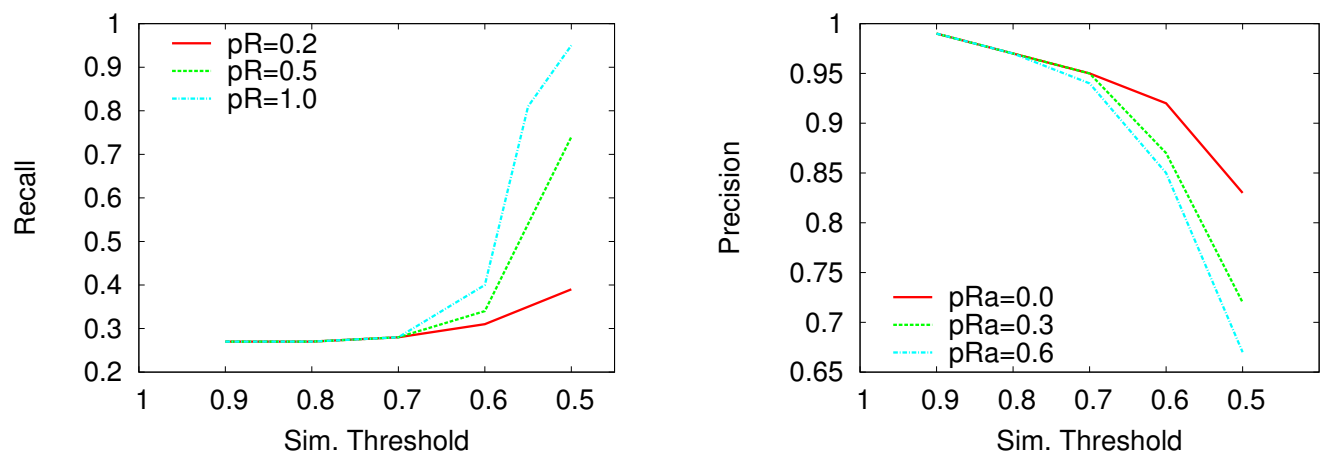

Figure 4.4: Effect of (a) identifying relations on recall and (b) ambiguous relations on precision for collective clustering

\subsubsection{Experiments using Synthetic Data}

I also experiment with synthetically generated data where I can control the structural characteristics. For this purpose, I used the two-stage data generator described in Appendix A to control the fraction of ambiguous and identifying relationships.

In the first set of experiments on synthetic data, I experiment with identifying relationships. I generate 500 co-occurrence relations from the same 100 entities with 200 entity-entity relationships using varying probability of co-occurrences $p^{R}=$ $0.2,0.5,1.0$ in the data. The probability of ambiguous relationships is held fixed, so that higher $p^{R}$ translates to higher probability of identifying co-occurrences in the data. Figure 4.4(a) shows recall at different similarity thresholds for three different co-occurrence probabilities. The results confirm that recall increases progressively with more identifying relationships at all thresholds.

In my second experiment, I consider the effect of ambiguous relations on precision of collective relational clustering. I add 200 binary relationships be- 

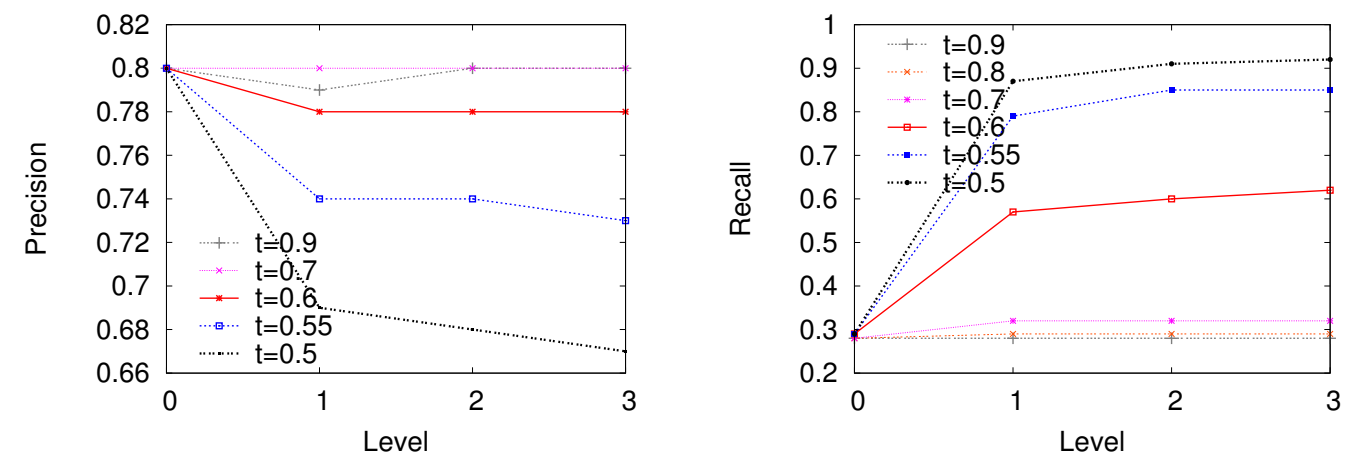

Figure 4.5: Change in (a) precision and (b) recall for increasing levels of cooccurrences used for collective clustering

tween 100 entities in three stages with increasing ambiguous relationship probability $\left(p_{a}^{R}=0,0.3,0.6\right)$. Then I perform collective clustering on 500 co-occurrence relations generated from each of these three settings. In Figure 4.4(b) I plot precision at different similarity threshold for three different values of $p_{a}^{R}$. The plots confirm the progressive decrease in precision for all thresholds with higher $p_{a}^{R}$. For both experiments, the results are averaged over 200 different runs.

Next, I move on to localized collective clustering. The numbers in Table 4.1 showed the converging nature of performance over increasing levels for queries on real datasets. I next resorted to synthetic data to verify this trend. In each run, I generated 2500 co-occurrence relations from 500 entities with an average of 2 neighbors per entity. Then I performed localized collective clustering in each case with the most ambiguous attribute value that corresponds to the highest number of underlying entities. In Figure 4.4(c) and (d), we see how precision and recall changes with increasing expansion level for a query. Precision goes down and recall goes up with increasing level, as is predicted by the analysis. The rate of increase/decrease 
depends on the structural properties. In other experiments, we have seen different rates of change, but the trend remains the same. The analysis also showed that precision and recall converges quickly depending on the structural properties. This too is confirmed by the two plots where the curves flatten out by level 3 .

As I have already discussed, an important issue with the collective relational clustering algorithm is the determination of the termination threshold. Note that this is an issue for all of the baselines as well, and here I report best accuracy over all thresholds. This is an area of ongoing research. Preliminary experiments have shown that the best threshold is query specific — setting the threshold depending on the ambiguity of the query results in significantly better accuracy than a fixed threshold for all queries. For an empirical evaluation, I cleaned the entire arXiv dataset offline by running $\mathbf{C R}$ on all its references together and terminated at the threshold that maximizes resolution accuracy over all references. This results in an overall accuracy (F1) of 0.98 . However, the average accuracy measured over the 75 queries in the test set is only 0.87. In comparison, recall that the best obtainable accuracy when resolving the queries individually each with a different threshold is 0.97. This suggests that there may be potential benefits to localized cleaning over its global counterpart in the offline setting.

\subsection{Conclusions}

In this chapter, I have motivated the problem of query-time entity resolution for accessing unresolved third-party databases. The biggest issue in query-time res- 
olution of entities is reducing the computational expense of collective resolution, while maintaining its benefits in terms of resolution accuracy. I have motivated query-time entity resolution as a constrained-resource problem and proposed an adaptive strategy for extracting the set of most relevant references for collectively resolving a query. I have formally analyzed the dependence of this approach on structural and other characteristics of the data to show analytically why a recursive expand-and-resolve strategy is feasible for this problem. I have demonstrated that this adaptive strategy preserves the accuracy of unconstrained expansion while dramatically reducing the number of relevant references, thereby enabling collective resolution at query-time. I have also demonstrated how the same adaptive strategy allows us to allocate resources depending on the ambiguity of the query, and reduces processing time for less ambiguous queries by 35\%. I have additionally performed extensive experiments on real and synthetic data to validate the trends predicted by the analysis. While I have presented results for bibliographic data, the techniques are applicable in other relational domains. Interesting directions of future research include exploring stronger coupling between the extraction and resolution phases of query processing and investigating localized resolution for offline data cleaning as well. 


\section{Chapter 5}

\section{Word Sense Disambiguation Using Bilingual Probabilistic Models}

In this chapter, I look at a potential application of entity resolution in the domain of natural language processing and consider the related problem of word sense disambiguation. The rest of the chapter is organized as follows. In Section 5.1, I briefly introduce the word sense disambiguation problem and discuss related work. In Section 5.2, I introduce the two probabilistic generative models for word sense disambiguation and describe how I construct the structure of the models in Section 5.3 and how the model parameters are trained in Section 5.4. I then discuss experimental results in Section 5.5 and analyze the performance of the two models in Section 5.6.

\subsection{Word Sense Disambiguation: Introduction and Related Work}

As mentioned in the introduction, the word sense disambiguation problem closely resembles the general definition of entity resolution when the senses are considered as entities and words in natural language documents as noisy observations

of the senses. However, it also presents some challenges that are unique to this domain. Carefully constructed sense ontologies for languages, such as the WordNet hierarchy for English, provide a valuable resource that approaches for solving this 
problem should take into account. Also, parallel corpora have words from multiple languages, all of which can be potentially ambiguous but the actual senses can differ from one language to another. Models that I develop for multi-lingual word sense disambiguation therefore need to resolve senses for different languages differently but, at the same time, need to construct cross-lingual relationships between senses. These are the challenges that I address in this chapter.

Word sense disambiguation (WSD) has been a central question in the computational linguistics community since its inception. WSD is fundamental to natural language understanding and is a useful intermediate step for many other language processing tasks [56]. Many recent approaches make use of ideas from statistical machine learning; the availability of shared sense definitions (e.g. WordNet [43]) and recent international competitions [61] have enabled researchers to compare their results. Supervised approaches $[23,105]$ typically outperform unsupervised approaches $[2,70,69,90,104,106]$, but often tend to be tuned to a specific corpus and are constrained by scarcity of labeled data.

In an effort to overcome the difficulty of finding sense-labeled training data, researchers have investigated unsupervised approaches for word sense disambiguation. Yarowsky [104] makes use of a thesaurus to find the most predictive words for each context. Yarowsky [106] proposes an iterative classification algorithm that uses the idea of 'one sense per collocation' to sense-tag all occurrences of a word starting from an initial set of labeled samples. Employing a similar idea, Mihalcea [75] uses non-ambiguous words to iteratively determine classes for ambiguous words. McCarthy et al. [74] make use of a thesaurus acquired from raw textual corpora and 
WordNet similarity to automatically find the most prevalent sense for each word in a document. Resnik [90] proposes a statistical measure of selectional preference of predicates towards specific classes or senses.

It has been pointed out that the use of parallel corpora for sense tagging can help with word sense disambiguation $[22,33,34,55,91]$. Brown et al. [22] use a flip-flop algorithm to split English words French words into two classes (senses) to have high mutual information through translations. Dagan and Itai [34] consider the disambiguation problem of finding the right target word in machine translation using a bilingual lexicon. They use statistics computed over corpora in the target language to choose the most likely translations for syntactic tuples (related words) in the source language. Ide [55] performs a statistical study of how the mapping of words to senses varies between different languages. Resnik and Yarowsky [91] suggest limiting sense distinctions to only those that are translated differently in different languages and propose cross-linguistic measures of sense distinction.

The main inspiration for my work is Diab and Resnik [38], who use relational structure in the form of translations for disambiguation and automatic sense tagging. Bengio and Kermorvant [5] present a graphical model that is an attempt to probabilistically formalize the main ideas of Diab and Resnik. Here I present two variants of the probabilistic model by Bengio and Kermorvant that incorporate relational structure to resolve senses. I also present empirical word sense disambiguation results which demonstrate the gain brought by this probabilistic relational approach, even while using only the translated word to provide disambiguation information. This is an instance where I do not make use of any attribute information for re- 
solving entities. They are resolved based on relational structure alone. However, the possible set of entity labels is provided to us in this case through the use of the WordNet hierarchy.

The first generative model, the Sense Model, does not model groups of senses explicitly. Instead, the senses in the two languages depend directly on each other in this model. Specifically, the Sense Model clusters semantically related words from the both languages into one set of senses, and translations are generated by probabilistically choosing a sense and then words from the sense. I show that this improves on the results of Diab and Resnik.

The next model, which I call the Concept Model, aims to improve on this by modeling the senses of the two languages separately and by relating senses from the two languages through a higher-level, semantically less precise concept. I use the term concept to denote a group of related senses. The concept plays the same role for word sense disambiguation as that of entity groups for resolution in Chapter 3. The intuition is that not all of the senses that are possible for a word will be relevant for a concept. In other words, the distribution over the senses of a word given a concept can be expected to have a lower entropy than the distribution over the senses of the word in the language as a whole. In this work, I look at translation data as a resource for identification of semantic concepts. Improved performance over the Sense Model validates the use of concepts in modeling translations.

For the rest of this chapter, for simplicity I will refer to the primary language of the parallel document as English and to the secondary as Spanish. 


\subsection{Probabilistic Models for Parallel Corpora}

I motivate the use of a probabilistic model by illustrating that disambiguation using translations is possible even when a word has a unique translation. For example, according to WordNet, the word prevention has two senses in English, which may be abbreviated as hindrance (the act of hindering or obstruction) and control (by prevention, e.g. the control of a disease). It has a single translation in the corpus, that being prevención. The first English sense, hindrance, also has other words such as bar that occur in the corpus and all of these other words are observed to be translated in Spanish as the word obstrucción. In addition, none of these other words translate to prevención. So it is not unreasonable to suppose that the intended sense for prevention when translated as prevención is different from that of bar. Therefore, the intended sense is most likely to be control. At the very heart of the reasoning is probabilistic analysis and independence assumptions. The assumption is that senses and words have certain occurrence probabilities and that the choice of the word can be made independently once the sense has been decided. This is the flavor that I look to add to modeling parallel documents for sense disambiguation. I formally describe the two generative models that use these ideas in Subsection 5.2.2 and Subsection 5.2.3.

\subsubsection{Notation}

Throughout this chapter, I use uppercase letters to denote random variables and lowercase letters to denote specific instances of the random variables. A trans- 


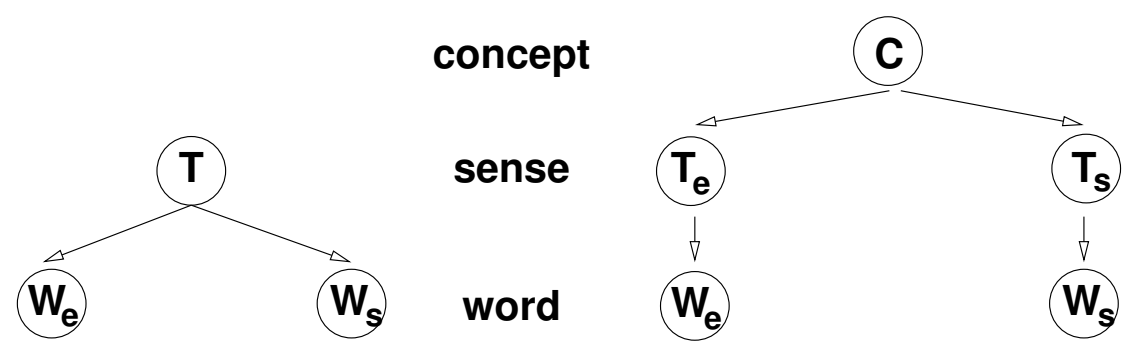

a) Sense Model

b) Concept Model

Figure 5.1: Graphical Representations of the a) Sense Model and the b) Concept Model

lation pair is $\left(W_{e}, W_{s}\right)$ where the subscript $e$ and $s$ indicate the primary language (English) and the secondary language (Spanish). $W_{e} \in\left\{w_{e_{1}}, \ldots, w_{e_{n}}\right\}$ and $W_{s} \in\left\{w_{s_{1}}, \ldots, w_{s_{m}}\right\}$. I use the shorthand $P\left(w_{e}\right)$ for $P\left(W_{e}=w_{e}\right)$.

\subsubsection{The Sense Model}

The Sense Model makes the assumption that the English word $W_{e}$ and the Spanish word $W_{s}$ in a translation pair share the same precise sense. In other words, the set of sense labels for the words in the two languages is the same and may be collapsed into one set of senses that is responsible for both English and Spanish words. Thus there is a one-to-one correspondence between the senses in the two languages and the single latent variable in the model is the sense label $T \in\left\{t_{1}, \ldots, t_{k}\right\}$ for both words $W_{e}$ and $W_{s}$. I also make the assumption that the words in both languages are conditionally independent given the sense label. The generative parameters $\theta_{g}$ for the model are the prior probability $P(t)$ of each sense $t$ and the conditional probabilities $P\left(w_{e} \mid t\right)$ and $P\left(w_{s} \mid t\right)$ of each word $w_{e}$ and $w_{s}$ in the two languages given the sense. The generation of a translation pair by this model may 
be viewed as a two-step process that first selects a sense according to the priors on the senses and then selects a word from each language using the conditional probabilities for that sense. This may be imagined as a factoring of the joint distribution: $P\left(W_{e}, W_{s}, T\right)=P(T) P\left(W_{e} \mid T\right) P\left(W_{s} \mid T\right)$. Note that in the absence of labeled training data, two of the random variables $W_{e}$ and $W_{s}$ are observed, while the sense variable $T$ is not. However, I can derive the possible values for the sense labels from WordNet, which gives us the possible senses for each English word $W_{e}$. The Sense model is shown in Figure 5.1(a).

\subsubsection{The Concept Model}

In the Sense Model, I avoided modeling the groups of senses explicitly. But the assumption of a one-to-one association between sense labels made in the Sense Model may be too simplistic to hold for arbitrary languages. In particular, it does not take into account that translation is from sentence to sentence (with a shared meaning), while the data being modeled are aligned single-word translations $\left(W_{e}, W_{s}\right)$, in which the intended meaning of $W_{e}$ does not always match perfectly with the intended meaning of $W_{s}$. Generally, a set of $m$ related senses in one language may be translated by one of $n$ related senses in the other. This many-to-many mapping is captured in the alternative model using a second level hidden variable called a concept. Thus there are three hidden variables in the Concept Model — the English sense $T_{e}$, the Spanish sense $T_{s}$ and the concept $C$, where $T_{e}=\left\{t_{e_{1}}, \ldots, t_{e_{k}}\right\}$, $T_{s}=\left\{t_{s_{1}}, \ldots, t_{s_{j}}\right\}$ and $C=\left\{c_{1}, \ldots, c_{l}\right\}$. 
I make the assumption that the senses $T_{e}$ and $T_{s}$ are independent of each other given the shared concept $C$. The generative parameters $\theta_{g}$ in the model are the prior probabilities $P(c)$ over the concepts, the conditional probabilities $P\left(t_{e} \mid c\right)$ and $P\left(t_{s} \mid c\right)$ for the English and Spanish senses given the concept, and the conditional probabilities $P\left(w_{e} \mid t_{e}\right)$ and $P\left(w_{s} \mid t_{s}\right)$ for the words $w_{e}$ and $w_{s}$ in each language given their senses. We can now imagine the generative process of a translation pair by the Concept Model as first selecting a concept according to the priors, then a sense for each language given the concept, and finally a word for each sense using the conditional probabilities of the words. This generative procedure may be captured by factoring the joint distribution using the conditional independence assumptions as $P\left(W_{e}, W_{s}, T_{e}, T_{s}, C\right)=P(C) P\left(T_{e} \mid C\right) P\left(W_{e} \mid T_{e}\right) P\left(T_{s} \mid C\right) P\left(W_{s} \mid T_{s}\right)$. The Concept model is shown in Figure 5.1(b).

\subsection{Constructing the Senses and Concepts}

Building the structure of the model is crucial for this task. Choosing the dimensionality of the hidden variables by selecting the number of senses and concepts, as well as taking advantage of prior knowledge to impose constraints, are very important aspects of building the structure.

If certain words are not possible for a given sense, or certain senses are not possible for a given concept, their corresponding parameters should be 0. For instance, for all words $w_{e}$ that do not belong to a sense $t_{e}$, the corresponding parameter $\theta_{w_{e} \mid t_{e}}$ would be 0 . Only the remaining parameters need to be modeled explicitly. 

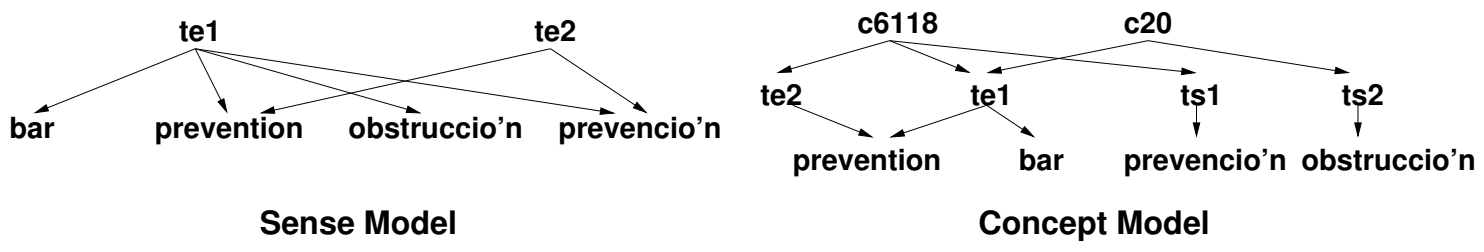

Figure 5.2: The Sense and Concept models for prevention, bar, prevención and obstrucción

While model selection is an extremely difficult problem in general, an important and interesting option is the use of world knowledge. Semantic hierarchies for some languages have been built. It should be possible to make use of these known taxonomies in constructing the model. I make heavy use of the WordNet ontology to assign structure to both the models, as I discuss in the following subsections. There are two major tasks in building the structure — determining the possible sense labels for each word, both English and Spanish, and constructing the concepts, which involves choosing the number of concepts and the probable senses for each concept. Instead of following the computationally challenging probabilistic approach of searching over the likelihood space of possible structures, I resort to the relational clustering approach from Subsection 2.3.3 to construct the senses and concepts.

\subsubsection{Building the Sense Model}

Each word in WordNet can belong to multiple synsets in the hierarchy, which are its possible senses. In both models, I directly use the WordNet senses as the English sense labels. All WordNet senses for which a word has been observed in the 
corpus form the set of English sense labels. The Sense Model holds that the sense labels for the two domains are the same. So the same WordNet labels must be used for the Spanish words as well. I include a Spanish word $w_{s}$ for a sense $t$ if $w_{s}$ is the translation of any English word $w_{e}$ in $t$.

\subsubsection{Building the Concept Model}

Unlike the Sense Model, the Concept Model does not constrain the Spanish senses to be the same as the English ones. So the two major tasks in building the Concept Model are constructing the Spanish senses and then clustering the English and Spanish senses to build the concepts.

Each Spanish word $w_{s}$ has its set of English translations $\left\{w_{e_{1}}, \ldots, w_{e_{k}}\right\}$. One possibility is to group Spanish words looking at their translations. However, a more robust approach is to consider the relevant English senses for $w_{s}$. Each English translation for $w_{s}$ has its set of English sense labels $S_{w_{e_{i}}}$ drawn from WordNet. So the relevant English sense labels for $w_{s}$ may be defined as $S_{w_{s}}=\bigcup_{i} S_{w_{e_{i}}}$. From the definition of neighborhood from Subsection 2.3.3, this is the English sense neighborhood $\operatorname{Nbr}_{S_{e}}\left(w_{s}\right)$ of the Spanish word $w_{s}$. I assume that different combinations of English senses define Spanish senses and I use the English sense neighborhood to define the Spanish sense(s) for any Spanish word $w_{s}$.

Each Spanish word may belong to one or more Spanish senses. If each word has a single sense, then I add a Spanish sense $t_{s}$ for each distinct English sense neighborhood, and all Spanish words having that neighborhood belong to that sense. 
Otherwise, each English neighborhood can be imagined to combine multiple Spanish senses. So I split the neighborhoods into frequently occurring subgroups or subneighborhoods. I identify sub-neighborhoods by intersecting pairs of neighborhoods and each sub-neighborhood that occurs significantly many times is recognized to represent a Spanish sense. I consider both ways of building Spanish senses. In either case, a constructed Spanish sense $t_{s}$ comes with its neighborhood of English senses, which I denote as $\mathrm{Nbr}_{S_{e}}\left(t_{s}\right)$.

Once I have the Spanish senses, I cluster them to form concepts. I use the English sense neighborhood corresponding to each Spanish sense to define a measure of similarity for a pair of Spanish senses. Following Subsection 2.3.3, I use the neighborhood similarity measure. The specific measure that I use is the Jaccard similarity, which is the most robust ${ }^{1}$. This similarity measure is then used to cluster the Spanish senses. Two Spanish senses are considered to be similar if their similarity measure is above a threshold. Then I take a transitive closure over these similarities to construct clusters of similar Spanish senses.

Finally, I build the concepts from the Spanish sense clusters. Since a concept is defined by a set of related English and Spanish senses, each Spanish sense cluster can be taken to represent a concept. This is because the Spanish senses in a cluster are grouped by similarity in their relations with English senses. So a concept is formed by taking the Spanish senses from each cluster and their related English senses. The

\footnotetext{
${ }^{1}$ Another option would be to use a measure of similarity for English senses, proposed by Resnik [89] for two synsets in a concept hierarchy like WordNet. The initial results with this measure were not favorable.
} 
relevant English senses come from the union of the English sense neighborhoods of all the Spanish senses in the cluster.

\subsection{Learning the Model Parameters}

Given the structure of the model, I use the popular EM algorithm [36] for hidden variables to learn the parameters for both models. The algorithm repeatedly iterates over two steps. The first step maximizes the expected log-likelihood of the joint probability of the observed data with the current parameter settings $\theta_{g}$. The next step then re-estimates the values of the parameters of the model. Below I summarize the re-estimation steps for each model.

\subsubsection{EM for the Sense Model}

The probability for each sense in the Sense Model and the probability for each English word given a sense can be re-estimated as:

$$
\begin{gathered}
P\left(T_{i}=t\right)=\frac{1}{N} \sum_{i=1}^{N} P\left(T=t \mid w_{e_{i}}, w_{s_{i}}, \theta_{g}\right) \\
P\left(W_{e_{i}}=e \mid T_{i}=t\right)=\frac{\sum_{w_{i}=e, i=1}^{N} P\left(T=t \mid w_{e_{i}}, w_{s_{i}}, \theta_{g}\right)}{\sum_{e} \sum_{W_{e_{i}}=e, i=1}^{N} P\left(T=t \mid w_{e_{i}}, w_{s_{i}}, \theta_{g}\right)}
\end{gathered}
$$

The probability $P\left(W_{s_{i}}=s \mid T_{i}=t\right)$ for each Spanish word given a sense follows similarly. 


\subsubsection{EM for the Concept Model}

The probabilities that need to be re-estimated for the Concept Model are the prior probability for each concept, the probability for each sense given a concept and the probability for each word given a sense. Now the sense are different in the two languages. So the prior for each concept, the probability for each English sense given a concept and the probability for each English word given an English sense can be re-estimated as follows:

$$
\begin{gathered}
P\left(C_{i}=k\right)=\frac{1}{N} \sum_{i=1}^{N} P\left(C_{i}=k \mid w_{e_{i}}, w_{s_{i}}, \theta_{g}\right) \\
P\left(T_{e_{i}}=l \mid C_{i}=k\right)=\frac{\sum_{i=1}^{N} P\left(C_{i}=k, T_{e_{i}}=l \mid w_{e_{i}}, w_{s_{i}}, \theta_{g}\right)}{\sum_{i=1}^{N} P\left(C_{i}=k \mid w_{e_{i}}, w_{s_{i}}, \theta_{g}\right)} \\
P\left(W_{e_{i}}=e \mid T_{e_{i}}=l\right)=\frac{\sum_{W_{e_{i}}=e, i=1}^{N} P\left(T_{e_{i}}=l \mid w_{e_{i}}=e, w_{s_{i}}, \theta_{g}\right)}{\sum_{e} \sum_{W_{e_{i}}=e, i=1}^{N} P\left(T_{e_{i}}=l \mid W_{e_{i}}=e, w_{s_{i}}, \theta_{g}\right)}
\end{gathered}
$$

The probabilities $P\left(T_{s_{i}}=m \mid C_{i}=k\right)$ and $P\left(W_{s_{i}}=s \mid T_{s_{i}}=m\right)$ for Spanish senses and words follow similarly.

\subsubsection{Initialization of Model Probabilities}

Since the EM algorithm performs gradient ascent as it iteratively improves the log-likelihood, it is prone to getting caught in local maxima, and selection of the initial conditions is crucial for the learning procedure. Instead of opting for a uniform or random initialization of the probabilities, I make use of prior knowledge about the English words and senses available from WordNet. WordNet provides occurrence frequencies for each synset in the SemCor Corpus that may be normalized 
to derive probabilities $P_{w n}\left(t_{e}\right)$ for each English sense $t_{e}$. For the Sense Model, these probabilities form the initial priors over the senses, while all English (and Spanish) words belonging to a sense are initially assumed to be equally likely. However, initialization of the Concept Model using the same knowledge is non-trivial. Each English sense $t_{e}$ should have $P_{\text {init }}\left(t_{e}\right)=P_{w n}\left(t_{e}\right)$. But since each sense belongs to multiple concepts, the constraint $\sum_{t_{e} \in c} P\left(t_{e} \mid c\right)=1$ also has to be satisfied for each concept. Instead of going for an optimal assignment, I settle for a compromise. I set $P_{\text {init }}\left(t_{e} \mid c\right)=P_{w n}\left(t_{e}\right)$ and $P(c)=\sum_{t_{e} \in c} P_{w n}\left(t_{e}\right)$. Subsequent normalization takes care of the sum constraints. For a Spanish sense, I set $P\left(t_{s}\right)=\sum_{t_{e} \in N b r_{S e}}\left(t_{s}\right) P_{w n}\left(t_{e}\right)$. Once I have the Spanish sense probabilities, I follow the same procedure for setting $P\left(t_{s} \mid c\right)$ for each concept. All the Spanish and English words for a sense are initially set to be equally likely, as in the Sense Model.

\subsection{Experimental Evaluation}

Both the models are generative probabilistic models learned from parallel corpora and are expected to fit the training and subsequent test data. A good fit should be reflected in good prediction accuracy over a test set. The prediction task of interest is the sense of an English word when its translation is provided. I estimate the prediction accuracy and recall of the models on Senseval data. ${ }^{2}$ In addition, the Concept Model learns a sense structure for the Spanish language. While it is hard

\footnotetext{
${ }^{2}$ Accuracy is the ratio of the number of correct predictions and the number of attempted predictions. Recall is the ratio of the number of correct predictions and the size of the test set.
} 
to objectively evaluate the quality of such a structure, I present some interesting concepts that are learned as an indication of the potential of this approach.

\subsubsection{Evaluation with Senseval Data}

In the experiments with real data, I make use of the parallel corpora constructed by Diab and Resnik [38] for evaluation purposes. I chose to work on these corpora in order to permit a direct comparison with their results. The sense-tagged portion of the English corpus is comprised of the English "all-words" section of the SENSEVAL-2 test data. The remainder of this corpus is constructed by adding the Brown Corpus, the SENSEVAL-1 corpus, the SENSEVAL-2 English Lexical Sample test, trial and training corpora and the Wall Street Journal sections 18-24 from the Penn Treebank. This English corpus is translated into Spanish using two commercially available MT systems: Globalink Pro 6.4 and Systran Professional Premium. The GIZA++ implementation of the IBM statistical MT models was used to derive the most-likely word-level alignments, and these define the English/Spanish word co-occurrences. To take into account variability of translation, I combine the translations from the two systems for each English word, following in the footsteps of Diab and Resnik [38]. For my experiments, I focus only on nouns, of which there are 875 occurrences in the tagged data. The sense tags for the English domain are derived from the WordNet 1.7 inventory. After pruning stopwords, I end up with 16,186 English words, 31,862 Spanish words and 2,385,574 instances of 41,850 distinct translation pairs. The English words come from 20,361 WordNet senses. 
Table 5.1: Comparison with Diab's Model

\begin{tabular}{|l|c|c|c|}
\hline & Accuracy & Recall & \# Parameters \\
\hline \hline Diab's Model & 0.618 & 0.572 & - \\
Sense Model & 0.624 & 0.616 & 154,947 \\
Concept Model & 0.672 & 0.651 & 120,268 \\
\hline
\end{tabular}

As can be seen from Table 5.1, both the models clearly outperform Diab [37], which is an improvement over Diab and Resnik [38], in both accuracy and recall, while the Concept Model does significantly better than the Sense Model with fewer parameters. The comparison is restricted to the same subset of the test data. For the best results, the Sense Model has 20,361 senses, while the Concept Model has 20,361 English senses, 11,961 Spanish senses and 7,366 concepts. The Concept Model results are for the version that allows multiple senses for a Spanish word. Results for the single-sense model are similar.

In Figure 5.3, I compare the prediction accuracy and recall against those of the 21 Senseval-2 English All Words participants and that of Diab [37], when restricted to the same set of noun instances from the gold standard. It can be seen that the proposed models outperform all the unsupervised approaches in recall and many supervised ones as well. No unsupervised approach is better in both accuracy and recall. It needs to be kept in mind that I take into account only bilingual data for predictions, and not monolingual features like context of the word as most other WSD approaches do. 


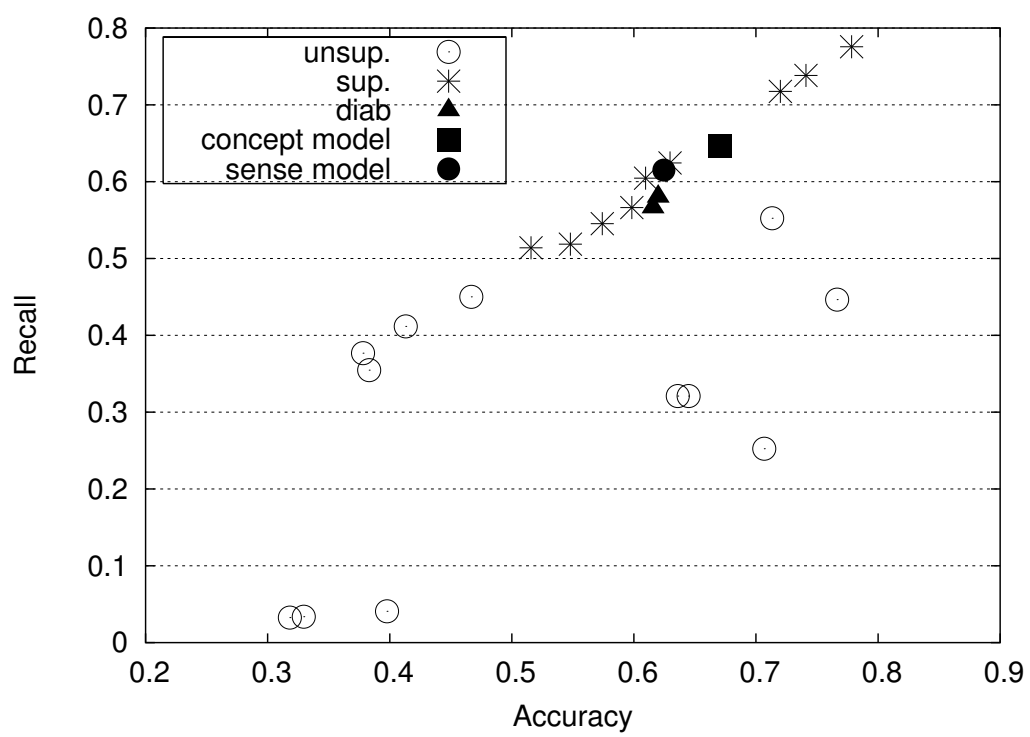

Figure 5.3: Comparison with Senseval2 WSD Systems

\subsubsection{Semantic Grouping of Spanish Senses}

An interesting by-product of the Concept Model is a semantic group structure for the senses in the secondary language. Table 5.2 shows some interesting examples of different Spanish senses for discovered concepts. ${ }^{3}$

The context of most concepts, like the ones shown, can be easily understood. For example, the first concept is about government actions and the second deals with murder and accidental deaths. The penultimate concept is interesting because it deals with different kinds of association and involves three different senses containing the word conexion. The other words in two of these senses suggest that they are about union and relation respectively. The third probably involves the link sense of connection. Conciseness of the concepts depends on the similarity threshold that is

\footnotetext{
${ }^{3}$ Some English words are found to occur in the Spanish Senses. This is because the machine translation system used to create the Spanish document left certain words untranslated.
} 
Table 5.2: Example Spanish Senses in a Concept. For each concept, each row is a separate sense. Dictionary senses of Spanish words are provided in English within parenthesis where necessary.

\begin{tabular}{|c|c|}
\hline $\begin{array}{l}\text { actos } \\
\text { supremas } \\
\text { decisión decisiones } \\
\text { gobernando gobernante } \\
\text { gubernamentales } \\
\text { gobernación gobierno-proporciona } \\
\text { prohibir prohibiendo prohibitivo prohibitiva } \\
\text { gubernamental gobiernos }\end{array}$ & $\begin{array}{l}\text { accidente accidentes } \\
\text { muertes(deaths) } \\
\text { casualty } \\
\text { matar(to kill) matanzas(slaughter) muertes-le } \\
\text { slaying } \\
\text { derramamiento-de-sangre (spilling-of-blood) } \\
\text { cachiporra(bludgeon) obligar(force) obligando(forcing) } \\
\text { asesinato(murder) asesinatos }\end{array}$ \\
\hline $\begin{array}{l}\text { linterna-eléctrica linterna(lantern) } \\
\text { faros-automóvil(headlight) } \\
\text { linternas-portuarias(harbor-light) } \\
\text { antorcha(torch) antorchas antorchas-pino-nudo }\end{array}$ & $\begin{array}{l}\text { manía craze } \\
\text { culto( } \text { cult) cultos proto-senility } \\
\text { delirio delirium } \\
\text { rabias (fury) rabia farfulla(do hastily) }\end{array}$ \\
\hline $\begin{array}{l}\text { oportunidad oportunidades } \\
\text { ocasión ocasiones } \\
\text { riesgo(risk) riesgos peligro(danger }) \\
\text { destino sino(fate) } \\
\text { fortuna suerte(fate) } \\
\text { probabilidad probabilidades }\end{array}$ & $\begin{array}{l}\text { diferenciación } \\
\text { distinción distinciones } \\
\text { especialización } \\
\text { maestría (mastery) } \\
\text { peculiaridades particularidades peculiaridades-inglesas } \\
\text { especialidad especialidades }\end{array}$ \\
\hline $\begin{array}{l}\text { diablo (devil) diablos } \\
\text { dickens } \\
\text { heller } \\
\text { lucifer satan satanás }\end{array}$ & $\begin{array}{l}\text { modelo parangón } \\
\text { ideal ideales } \\
\text { santo(saint) santos san } \\
\text { idol idols ídolo }\end{array}$ \\
\hline $\begin{array}{l}\text { deslumbra }(\text { dazzle }) \\
\text { cromo }(\text { chromium }) \\
\text { meteoro meteoros meteor meteoros-blue } \\
\text { meteorito meteoritos } \\
\text { pedregosos }(\text { rocky })\end{array}$ & $\begin{array}{l}\text { dios god dioses } \\
\text { divinidad divinity } \\
\text { inmortal }(\text { immortal }) \text { inmortales } \\
\text { teología teolog } \\
\text { deidad deity deidades }\end{array}$ \\
\hline $\begin{array}{l}\text { variación variaciones } \\
\text { discordancia desacuerdo(discord) discordancias } \\
\text { desviación(deviation) desviaciones desviaciones-normales } \\
\text { discrepancia discrepancias fugaces(fleeting) variación diferencia } \\
\text { disensión }\end{array}$ & $\begin{array}{l}\text { minutos minuto } \\
\text { momento momentos un-momento } \\
\text { minutos momentos momento segundos } \\
\text { instante momento } \\
\text { pestañeo(blink) guiña }(\text { wink }) \text { pestañean }\end{array}$ \\
\hline $\begin{array}{l}\text { adhesión adherencia ataduras }(\text { tying) } \\
\text { enlace( connection) ataduras } \\
\text { atadura ataduras } \\
\text { conexión conexiones } \\
\text { conexión une(to unite) } \\
\text { relación conexión } \\
\text { implicación (complicity) envolvimiento }\end{array}$ & $\begin{array}{l}\text { pasillo(corridor) } \\
\text { aisle } \\
\text { pasarela(footbridge) } \\
\text { hall vestíbulos } \\
\text { pasaje(passage) } \\
\text { callejón(alley) callejas-ciegas (blind alley) callejones-ocultos }\end{array}$ \\
\hline
\end{tabular}


selected. Some may bring together loosely-related topics, which can be separated by a higher threshold.

\subsection{Model Analysis}

In this section, I back up the experimental results with an in-depth analysis of the performance of the two proposed models.

The Sense Model was motivated by Diab and Resnik [38] but the flavors of the two are quite different. The most important distinction is that the Sense Model is a probabilistic generative model for parallel corpora, where interaction between different words stemming from the same sense comes into play, even if the words are not related through translations, and this interdependence of the senses through common words plays a role in sense disambiguation.

I started off with the discussions on semantic ambiguity with the intuition that identification of semantic concepts in the corpus that relate multiple senses should help disambiguate senses. The Sense Model falls short of this target since it only brings together a single sense from each language. I will now revisit the motivating example from Section 5.2 and see how concepts help in disambiguation by grouping multiple related senses together.

For the Sense Model, $P\left(\right.$ prevention $\left.\mid t_{e_{2}}\right)>P\left(\right.$ prevention $\left.\mid t_{e_{1}}\right)$ since it is the only word that $t_{e_{2}}$ can generate. However, this difference is compensated for by the higher prior probability $P\left(t_{e_{1}}\right)$, which is strengthened by both the translation pairs. Since the probability of joint occurrence is given by the product $P(t) P\left(w_{e} \mid t\right) P\left(w_{s} \mid t\right)$ for 
any sense $t$, the model does not develop a clear preference for any of the two senses.

The critical difference in the Concept Model can be appreciated directly from the corresponding joint probability $P(c) P\left(t_{e} \mid c\right) P\left(w_{e} \mid t_{e}\right) P\left(t_{s} \mid c\right) P\left(w_{s} \mid t_{s}\right)$, where $c$ is the relevant concept in the model. The preference for a particular instantiation in the model is dependent not on the prior $P\left(t_{e}\right)$ over a sense, but on the sense conditional $P\left(t_{e} \mid c\right)$. In the example, since <bar, obstrucción $>$ can be generated only through concept $c 20, P\left(t_{e_{1}} \mid c 20\right)$ is the only English sense conditional boosted by it. <prevention, prevención $>$ is generated through a different concept c6118, where the higher conditional $P\left(\right.$ prevention $\left.\mid t_{e_{2}}\right)$ gradually strengthens one of the possible instantiations for it, and the other one becomes increasingly unlikely as the iterations progress. The inference is that only one sense of prevention is possible in the context of the parallel corpus. The key factor in this disambiguation was that two senses of prevention separated out in two different concepts.

The other significant difference between the models is in the constraints on the parameters and the effect that they have on sense disambiguation. In the Sense Model, $\sum_{t} P(t)=1$, while in the Concept Model, $\sum_{t_{e} \in c} P\left(t_{e} \mid c\right)=1$ separately for each concept $c$. Now for two relevant senses for an English word, a slight difference in their priors will tend to get ironed out when normalized over the entire set of senses for the corpus. In contrast, if these two senses belong to the same concept in the Concept Model, the difference in the sense conditionals will be highlighted since the normalization occurs over a very small set of senses - the senses for only that concept, which in the best possible scenario will contain only the two contending senses, as in concept $c 118$ of the example. 
As can be seen from Table 5.1, the Concept Model not only outperforms the Sense Model, it does so with significantly fewer parameters. This may be counterintuitive since Concept Model involves an extra concept variable. However, the dissociation of Spanish and English senses can significantly reduce the parameter space. Imagine two Spanish words that are associated with ten English senses and accordingly each of them has a probability for belonging to each of these ten senses. Aided with a concept variable, it is possible to model the same relationship by creating a separate Spanish sense that contains these two words and relating this Spanish sense with the ten English senses through a concept variable. Thus these words now need to belong to only one sense as opposed to ten. Of course, now there are new transition probabilities for each of the eleven senses from the new concept node. The exact reduction in the parameter space will depend on the frequent subneighborhoods discovered for the English sense neighborhoods of the Spanish words. Longer and more frequent sub-neighborhoods will lead to larger reductions. It must also be borne in mind that this reduction comes with the independence assumptions made in the Concept Model.

It may be noted that predicting senses from translations need not necessarily be an end result in itself. As I have already mentioned, lack of labeled data is a severe hindrance for supervised approaches to word sense disambiguation. At the same time, there is an abundance of bilingual documents and many more can potentially be mined from the web. It should be possible using the proposed approach to (noisily) assign sense tags to words in such documents, thus providing huge resources of labeled data for supervised approaches to make use of. 


\section{Chapter 6}

\section{Related Work}

In this chapter, I review related work on entity resolution and group / topic models. I discusss related work for word sense dismabiguation in Section 5.1. The entity resolution problem has been studied in many different areas under different names - co-reference resolution, deduplication, object uncertainty, record linkage, reference reconciliation, etc. Here I review some of the main work, but the review is not exhaustive. Winkler [102] also provides a nice summary report.

\subsection{Approximate Matching}

The traditional approach to entity resolution looks at textual similarity in the descriptions of the entities. For example, determining whether two citations refer to the same paper depends on the similarity measure such as edit distance between the two citation strings. There has been extensive work on defining approximate string similarity measures $[78,80,30,27]$ that may be used for unsupervised entity resolution. Cardie and Wagstaff [24] have posed noun phrase coreference in natural language documents as an unsupervised clustering problem based on a similarity measure that combines multiple linguistic features. Another approach is to use adaptive supervised algorithms that learn string similarity measures from labeled 
data $[92,15,31,100]$. One of the difficulties in using a supervised method for resolution is constructing a good training set that includes a representative collection of positive and negative examples. Other approaches use active learning [94, 100], where the user is asked to label ambiguous examples by the learner.

\subsection{Theoretical Bounds for Cleaning}

Cohen et al. [29] studies the theoretical problem of 'hardening a soft database' that has many co-referent entries. Hardening refers to the task of figuring out which pairs of soft identifiers refer to the same real world object. Given the likelihood of being co-referent for each soft pair and a probability distribution of possible hard databases, hardening is defined as the optimization problem of finding the most likely hard model given the soft facts. A cost is associated with each hard tuple that is added to the database and for each co-reference decision made. They show that this optimization problem is NP-hard even under strong restrictions. They propose a greedy agglomerative clustering approach for an approximate solution. This algorithm's complexity is linear in the number of entries in the soft database.

\subsection{Efficiency Issues}

Given that solving the entity resolution problem optimally is computationally expensive, an important focus is on efficiency issues in data cleaning, where the goal is to come up with inexpensive algorithms for finding approximate solutions to the problem. The key mechanisms for doing this involve computing the matches 
efficiently and employing techniques commonly called 'blocking' to quickly find potential duplicates and eliminate non-duplicates from consideration [52, 79, 72, 54].

The merge/purge problem was posed by Hernandez and Stolfo [52] with efficient schemes to retrieve potential duplicates without resorting to quadratic complexity. They use a 'sorted neighborhood method' where an appropriate key is chosen for matching. Records are then sorted or grouped according to that key and potential matches are identified using a sliding window technique. However, some keys may be badly distorted so that their matches cannot be spanned by the window and such cases will not be retrieved. The solution proposed is a multi-pass method over different keys and then merging the results using transitive closure. Monge and Elkan [79] combine the union find algorithm with a priority queue look-up to find connected components in an undirected graph. McCallum et al. [72] propose the use of canopies to first partition the data into overlapping clusters using a cheap distance metric and then use a more accurate and expensive distance metric for those data pairs that lie within the same canopy. Gravano et al. [50] propose a sampling approach to quickly compute cosine similarity between tuples for fast text-joins within an SQL framework. Chaudhuri et al. [27] use an error tolerant index for data warehousing applications for probabilistically looking up a small set of candidate reference tuples for matching against an incoming tuple. This is considered 'probabilistically safe' since the closest tuples in the database will be retrieved with high probability. This is also efficient since only a small number of matches needs to be performed. Swoosh [6] has recently been proposed as a generic entity resolution framework that considers resolving and merging duplicates as a database 
operator and the goal is to minimize the number of record-level and feature-level operations.

\subsection{Probabilistic Models for Entity Resolution}

The groundwork for posing entity resolution as a probabilistic classification problem was done by [44], who extend the ideas of [83] for labeling pairs of records from two different files to be merged as "match" or "non-match" on the basis of agreement among their different fields. Winkler [103] and more recently Ravikumar and Cohen [88] have built upon this work.

Probabilistic models that take into account interaction between different entity resolution decisions have been proposed for named entity recognition in natural language processing and for citation matching. McCallum and Wellner [73] use conditional random fields for noun coreference and use clique templates with tied parameters to capture repeated relational structure. Singla and Domingos [98] use the idea of merging evidence to allow the flow of reasoning between different pair-wise decisions over multiple entity types. These two relational models are supervised and require labeled data to train the parameters. Wellner and McCallum have also extended their model for performing extraction and resolution jointly [101].

Availability of sufficient labeled data is often an issue for this problem and unsupervised relational models have also been developed. Li et al. [67] address the problem of disambiguating "entity mentions", potentially of multiple types, in the context of unstructured textual documents. They propose a probabilistic 
generative model that captures a joint distribution over pairs of entities in terms of co-mentions in documents. Pasula et al. [86] propose a generic probabilistic relational model framework for the citation matching problem. Daumé and Marcu [35] have recently proposed an extension to Pasula et al.'s model, where the number of clusters or entities is directly modeled by a Dirichlet Process and is similar in spirit to ours. However, I propose a three level model where the selection of author entities depends on the groups that they belong to. Milch et al. [76] propose a more general approach to the identity uncertainty problem. They present a formal generative language for defining probability distribution over worlds with unknown objects and identity uncertainty. This can be seen as a probability distribution over first order model structures with varying number of objects. They show that the inference problem is decidable for a large class of these models and propose a rejection sampling algorithm for estimating probabilities.

All of these probabilistic models have been shown to perform well in practice and have the advantage that the match / non-match decisions do not depend on any user specified threshold but are learned directly from data. However, this benefit comes at a price. Inference in relational probabilistic models is an expensive process. Exact inference is mostly intractable and approximate strategies such as loopy belief propagation and monte carlo sampling strategies are employed. Even these approximate strategies take several iterations to converge and extending such approaches to large datasets is still an open problem. 


\subsection{Non-probabilistic Relational Approaches}

Alternative approaches $[3,60,41]$ consider relational structure of the entities for data integration but avoid the complexity of probabilistic inference. By avoiding a formal probabilistic model, these approaches can handle complex relationships between different entities more easily and the resolution process is significantly faster as well.

Kalshnikov et al. [60] enhance feature-based similarity between an ambiguous reference and the many entity choices for it with relationship analysis between the entities, such as affiliation and co-authorship. They propose a 'content attraction principle' hypothesizing that an ambiguous reference will be more strongly connected via such relationships to its true entity compared to other entity choices for it. They translate this principle to a set of non-linear equations involving connection strengths in the entity graph, which are solved to determine the entity choice for each reference. This approach is useful for incremental data cleaning when the set of entities currently in the database is known and an incoming reference needs to be matched with one of these entities. In the more general setting that I consider, the entities are not known and need to be discovered.

Ananthakrishna et al. [3] introduce relational deduplication in data warehouse applications where there is a dimensional hierarchy over the relations. Using an approach similar in spirit to our naive relational baseline, they augment the string similarity measure between two tuples with the similarity between their foreign key relations across the hierarchy which they call children sets. In the specific case, 
where the relationships represent an ordered set as in a domain hierarchy, they show how the similarity computation can be made more efficient. To avoid comparison between all pairs of tuples in a relation, they propose a grouping strategy that makes uses of the relational hierarchy.

The approach that is the most similar in spirit to relational clustering algorithm is that of Dong et al. [41]. They collectively resolve entities of multiple types by propagating relational evidence in a dependency graph, and demonstrate the benefits of collective resolution in real datasets. Their approach creates a binary decision node for each potential pair of duplicates, which can be expensive in large datasets. One key strategy that they employ to propagate evidence is merging attribute decision nodes. Specifically, for a pair of names such as 'J. Smith' and 'John Smith', they create a single decision node in the dependency graph and multiple entity pairs can share this decision. However, while 'John Smith' and 'J. Smith' may refer to the same individual for a particular mention of ' $\mathrm{J}$ Smith', it is quite possible for another mention of 'J. Smith' to refer to 'James Smith'. This approach is useful for identifying many dispersed references for the same entity but not for domains where disambiguation is important.

Neville et al. [82] explore different graph partition schemes for clustering in graphs where the edge weights reflect attribute similarity between nodes. By varying the edge weights and edge existence probabilities conditioned on the cluster labels, they compare algorithms that consider only attributes and those that combine attribute and relational evidence. They report that spectral techniques for partitioning [97] work better that other min-cut and k-clustering approaches but 
combining attribute and relational information proves detrimental for clustering.

The SUBDUE system proposed by Jonyer et al. [59] is a scheme for conceptual clustering of structured data. In addition to partitioning the data, conceptual clustering also summarizes the clusters with conceptual descriptions of objects contained in them. SUBDUE generates a hierarchical conceptual clustering by discovering substructures in the data using the minimum description length principle. This helps to compress the graph and represent conceptual structure as well.

Doan et al. [40] explore a profiler-based approach for tying up disjoint attributes for sanity checks using domain knowledge. For example, on merging two objects, (9, John Smith) and (John Smith, 120k) from two tables with schemas (age, name) and (name, salary), we get a person whose age is 9 years and whose salary is 120K. This would be deemed an unlikely match by a profiler.

\subsection{Group and Topic Modeling}

Many models have been proposed over the last few years for discovering groups or topics (in the context of documents) from co-occurrences. Hofmann [53] proposed a probabilistic interpretation of latent semantic indexing (PLSI) as one of the first topic mixture models for documents. Cohn and Hofmann [32] later accounted for hyper-links to propose a joint model for words and links. Blei et al. [21] improved Hofmann's PLSI model by introducing a prior over topic mixtures in the Latent Dirichlet Allocation (LDA) model. One way to accommodate variable number of groups or mixture components is the Dirichlet Process mixture model [4]. An alter- 
native approach that leads to the same formalism is to derive the limit of a finite mixture model, where the number of components is taken to infinity [81]. Many extensions of the Dirichlet process mixture model have since been proposed, such as the hierarchical topic model [18], correlated topic models [20] and Pachinko Allocation [66] to account for correlations between different components.

Recent approaches have been proposed to incorporate author entities into topic models [93, 71]. Kubica et al. [63] have proposed a different style of generative models for links using underlying groups of entities. But none of these component mixture models account for uncertainty in the identity of the entities, though the author-topic model [93] recognizes the problem of duplicate authors. The only other mixture model approach to entity resolution, apart from ours, is that of Daume' and Marcu [35] who use the Dirichlet Process mixture model for resolving entities. However, they do not account for relationships among the entities.

Exact inference is known to be intractable for the topic/group mixture models and different approximate inference strategies are resorted to. Hofmann [53] uses annealed or tempered Expectation Maximization, while Blei et al. have proposed variational approaches [19]. Others [81, 51, 93] have developed approximate inference algorithms using sampling strategies. Split-merge inference has been recently proposed in the context of Metropolis-Hasting sampling by Jain and Neal $[57,58]$. I have proposed similar ideas for the LDA-ER model in the context of Gibbs Sampling inference. 


\subsection{Queries}

Little work has been done in the literature for query-centric cleaning or relational approaches for answering queries, where execution time is as important as accuracy of resolution. Approaches have been proposed for localized evaluation of Bayesian networks [42], but not for clustering problems. Recently, Chandel et al. [26] have addressed efficiency issues in computing top-k entity matches against a dictionary in the context of entity extraction from unstructured documents. They process top-k searches in batches where speed-up is achieved by sharing computation between different searches. Fuxman et al. [47] motivate the problem of answering queries over databases that violate integrity constraints and address scalability issues in resolving inconsistencies dynamically at query-time. However, the relational aspect of the problem, which is the major scalability issue that I address, does not come up in any of these settings.

\subsection{Data Cleaning Tools}

A number of frameworks and tools have also been developed for data cleaning. Galhardas et al. [46] propose a framework for declarative data cleaning by extending SQL with specialized operators for matching, clustering and merging. The WHIRL system [28] integrates a logical query language for doing 'soft' text joins in databases with efficient query processing. Potter's Wheel [87], Active Atlas [100] and D-Dupe [17] are some other data cleaning frameworks that involve user interaction. 


\subsection{Application Domains}

Data cleaning and reference disambiguation approaches have been applied and evaluated in a number of domains. The earliest application is on medical data [83]. Census data is an area where detection of duplicates poses a significant challenge and Winkler [103] has successfully applied his research and other baselines to this domain. A great deal of work has been done making use of bibliographic data $[54,65,72,94,86]$. Almost without exception, the focus has been on the matching of citations. Work in coreference resolution and disambiguating entity mentions in natural language processing $[73,67]$ has been applied to text corpora and newswire articles like the TREC corpus. There has been recent research on resolving entities in email [39] and geospatial data [96]. For detailed evaluation of algorithm perfor-

mance, researchers have also resorted to synthetic [82] and semi-synthetic [3, 27] datasets where various features of the data can be varied in a controlled fashion.

\subsection{Evaluation Metrics}

As has been pointed out by Sarawagi et al. [94], choice of a good evaluation metric is an issue for entity resolution tasks. Mostly, resolution has been evaluated as a pair-wise classification problem. Accuracy may not be the best metric to use since datasets tend to be highly skewed in their distribution over duplicate and non-duplicate pairs; often less than $1 \%$ of all pairs are duplicates. In such a scenario, a trivial classifier that labels all pairs as non-duplicates would have $99 \%$ accuracy. Though accuracy has been used by some researchers [103, 78, 27, 82], most have used precision over the duplicate prediction and recall over the entire set 
of duplicates. Observe that a classifier that indiscriminately labels all pairs as nonduplicates will have high precision but zero recall. The two measures are usually combined into one number by taking their harmonic mean. This is the so-called F1 measure. Another option that has been explored is weighted accuracy but this may report high accuracy values even when precision is poor. Cohen et al. [30] rank all candidate pairs by distance and evaluate the ranking. In addition to the maximum F1 measure of the ranking, they consider the non-interpolated average precision and interpolated precision at specific recall levels.

Some other approaches to this problem have posed it as a clustering task, where references that correspond to the same entity are associated with the same cluster. Performance measures that evaluate the qualities of the clusters generated compared to the true clusters are more relevant in such cases. Monge and Elkan [79] use a notion of cluster purity for evaluation. Each of the generated clusters may either match a true cluster, be a subset of a true cluster or include references from more than one cluster. They treat the first two cases as pure clusters while the third category of clusters is deemed impure. They use the number of pure and impure clusters generated as the evaluation metric. I have proposed an alternative evaluation metric for this clustering task where I measure the diversity of each constructed cluster of entity references in terms of the number of references to different real entities that it contains [8] and the dispersion of each entity over the number of different clusters. I show that dispersion-diversity plots capture the quality of the clusters directly and can be used to evaluate the trade-off in a fashion similar to precision-recall curves. 


\section{Chapter 7}

\section{Conclusions and Future Directions}

In this dissertation, I have defined the problem of collective relational entity resolution. I have demonstrated it to be a powerful and promising approach that combines attribute similarity with relational evidence and improves performance over traditional approaches. I have introduced two different approaches for collective entity resolution. The collective relational clustering approach combines attribute similarity with relational similarity and performs greedy agglomerative clustering to identify the underlying entities. While it is fast and intuitive in nature, performance depends on the choice of a termination threshold. My second approach, a probabilistic generative model for collective entity resolution, uses non-parametric Bayesian estimation to obviate the need for any user-specified threshold. The novelty in my model is the discovery of hidden group structures among the underlying entities for collective entity resolution. For both models, I have focused on designing efficient algorithms. Efficiency issues are addressed in the greedy relational clustering approach using indexed priority queues and indexes for keeping track of dependencies. For my probabilistic model for collective resolution, I have proposed an improved split-merge sampling algorithm for speeding up inference. Using extensive experiments, I have demonstrated that both models improve resolution performance over 
traditional approaches. I have also motivated the problem of query-time entity resolution for accessing unresolved third-party databases and have proposed adaptive algorithms for performing collective resolution at query-time. As an application of the entity resolution problem in natural language processing, I have studied the problem of word sense disambiguation and demonstrated that my models for sense disambiguation using translations improve performance over other unsupervised approaches.

There are many interesting avenues for future work. There are aspects in both the relational clustering algorithm and the LDA-ER model that require further research. In order for the relational clustering approach to be effective in practice, the termination threshold needs to be specified. The obvious way to automate this is to learn the threshold from labeled training examples. In this dissertation, I have focused on unsupervised approaches for entity resolution. But though manual tagging of training samples is an expensive process, it is possible to collect small numbers of labeled examples for training in most domains. Labeled examples can also be used for learning the parameters of the similarity measure — the weight $\alpha$ for combining relational and attribute similarity and also weights for different attributes that may be available for each domain. I have performed some preliminary experiments in this direction and found that even small amounts of labeled samples can be helpful. Finding the right examples to label for optimal benefit might be an interesting direction for research. It will also be interesting to investigate merge sequences other than the greedy approach and analyze the sensitivity of resolution performance to different merge sequences, though preliminary experiments have shown that the greedy 
strategy performs the best. The probabilistic generative model can be improved to handle more general attributes and to account for the words in the documents themselves. Currently, references in each paper are generated independently of each other given the group distribution. Instead, we may associate a generation styles with each paper that all references in the paper have to follow. For example, if one name in a paper is abbreviated in a particular way, the other names are most likely to be abbreviated following the same style. Additionally, there needs to be further research in more efficient inference algorithms for component models such as LDA-ER.

There are also several research direction for collective relational entity resolution in general. I have done preliminary work on extending my relational clustering algorithm to handle multiple types of entities [9]. Most real applications involve multiple entity types that relate to one another and further research needs to be done for this problem. There are several domains that present significant and unique challenges for collective entity resolution, such as handling spatial relationships in geospatial data integration and modeling the temporal aspect of relationships for resolving name references in emails. Applying collective resolution for matching nodes across ontologies also poses interesting challenges due to the hierarchical nature of the relationships.

An important focus in this dissertation has been on efficiency issues and I have proposed algorithms that scale nicely with the size of the data. But today it is common for real datasets to have millions of records. This brings up many issues that we have not addressed. For example, I have implicitly assumed that the datasets 
fit in memory. Significant research needs to be done for collective cleaning to work for web-scale datasets. An alternative to offline cleaning that I have explored in this dissertation is that of resolving entities on the fly as and when required. Based on my results, this seems to be a promising approach. Query processing time may be improved further by caching and reusing computation between different queries. Currently, my algorithm works in two distinct phases where the relevant references are extracted in the first phase and then resolved in the second. Response times may be improved by stronger coupling between the extraction and resolution phases, where expansions are done only when available evidence is insufficient for resolution.

In all of my approaches, I have assumed that the references and relationships have already been extracted and are available in a database. However, automated extraction from unstructured data is a very challenging problem and is a subject of ongoing research. Exploring joint models for extraction and resolution is another promising direction.

To summarize, the entity resolution problem is attracting growing attention to address the influx of structured and semi-structured data from a multitude of heterogeneous sources. Accurate resolution is important for a variety of reasons ranging from cost-effectiveness and reduction in data volume to accurate analysis for critical applications. In the case of structured data, it is especially important to look at entity resolution from a relational perspective. In this dissertation, I have explored the entity resolution problem and have demonstrated that approaches that consider relationships between different database records and make decisions collectively for related records significantly improve performance over traditional 
baselines. This dissertation is among the first to motivate and investigate the impact of collective relational clustering for a critical data integration problem. Looking into the future, as available data grows in volume and diversity, data analysis tasks get more complex, and accuracy of information becomes more critical than ever before, the significance and benefits of collective relational models for handling uncertainity in real database applications will only become more obvious. 


\section{Appendix A}

\section{Synthetic Data Generation}

In this appendix, I describe my approach for generating synthetic data that I have used for validating and analyzing the different models and algorithms that I have proposed in this dissertation. The synthetic data experiments are crucial for understanding different aspects of the proposed approaches. While experiments on real data are crucial for appreciating the impact of the approaches in real life scenarios, they do not provide a complete picture. As we have seen, the three different real datasets that we have used have varying characteristics, and performances of the algorithms differ from one dataset to another. This naturally leads to the following question — 'How well will the algorithms perform on a new dataset?'. In order to answer this question, I have analyzed the proposed approaches theoretically to quantify their dependence on different data characteristics. I have then relied on synthetic data to back up this analysis with empirical evidence.

Since I have proposed LDA-ER as a generative model for co-occurring references in this dissertation, it is worthwhile to explain why I do not use it as the synthetic data generator. First, while data generated using LDA-ER may be used for validating the other approaches, it will not be very illuminating for analyzing LDA-ER itself. Secondly, LDA-ER is not a 'complete' generative model, in that 
it does not model all aspects of the data. Some of the data characteristics, such as the number of references, the reference attributes, the number of co-occurrence relations, the size of the co-occurrence relations, etc., are observed and LDA-ER does not propose a generative model for these aspects. Also, LDA-ER does not directly model the sizes of the collaborative groups or the ambiguities of the entity attributes and relationships. All of these are important characteristics that the generative process for synthetic data needs to handle.

The different characteristics of the data can be broadly divided into two categories - properties of the underlying entities and their relationships, and properties of the generation process of the co-occurrence relationships. Examples from the first category are the number of entities, the ambiguity of the entity attributes, the number of relationships among the entities, etc, while the number of co-occurrence relations in the data, the average size of the co-occurrence relations and the reference attributes are examples from the second category. Accordingly, the synthetic data generation process has two stages. In the first stage, I create the collaboration graph among the underlying entities and the entity attributes. In the second, co-occurrence relations are generated from this collaboration graph. A high level description of the generative process in shown in Figure A.1. Next I describe the two stages of the generation process one by one.

The graph creation stage, in turn, has two sub-stages. First the domain entities and their attributes are created and then relationships are added between them. For creating entities, I control the number of entities and the ambiguity of their attributes. I create $N$ entities and their attributes one after another. For simplicity 


\section{Creation Stage}

1.

Repeat $\mathrm{N}$ times

2.

Create random attribute $x$ with ambiguity $p_{a}$

3.

Create entity $e$ with attribute $x$

4.

Repeat M times

5.

Choose entity $e_{i}$ randomly

6.

Choose entity $e_{j}$ with prob $p_{a}^{R}$ of an ambiguous relationship $\left(e_{i}, e_{j}\right)$

7.

Set $e_{i}=\operatorname{Nbr}\left(e_{j}\right)$ and $e_{j}=N b r\left(e_{i}\right)$

\section{Generation Stage}

8. $\quad$ Repeat R times

9.

Randomly choose entity $e$

10.

Generate reference $r$ using $\mathcal{N}(e . x, 1)$

11.

Initialize hyper-edge $h=\langle r\rangle$

12.

Repeat with probability $p_{c}$

13.

14.

Randomly choose $e_{j}$ from $\mathrm{Nbr}(e)$ without replacement

15.

Generate reference $r_{j}$ using $\mathcal{N}\left(e_{j} . x, 1\right)$

Add $r_{j}$ hyper-edge $h$

16.

Output hyper-edge $h$

Figure A.1: High-level description of synthetic data generation algorithm 
and without losing generality, each entity $e$ has a single floating point attribute e.x, instead of a character string. A parameter $p_{a}$ controls the ambiguity of the entity attributes; with probability $p_{a}$ the attribute of a new entity is chosen from values that are already in use by existing entities. Then $M$ binary relationships are added between the created entities. As with the attributes, there is a parameter controlling the ambiguity of the relationships, as defined in Section 4.3. For each binary relationship $\left(e_{i}, e_{j}\right)$, first $e_{i}$ is chosen randomly and then $e_{j}$ is sampled so that $\left(e_{i}, e_{j}\right)$ is an ambiguous relationship with probability $p_{a}^{R}$.

Before describing the process of generating co-occurrence relationships from the graph, let us consider in a little more detail the issue of attribute ambiguity. What finally needs to be controlled is the ambiguity of the reference attributes. While these depend on the entity attributes, they are not completely determined by entities. Taking the example of names, two people who have names 'John Michael Smyth' and 'James Daniel Smith' can still be ambiguous in terms of their observed names in the data depending on the generation process of observed names. In other words, attribute ambiguity of the references depends both on the separation between entity attributes and the dispersion created by the generation process. I make the assumption that for an entity $e$ with attribute $e . x$, its references are generated from a Gaussian distribution with mean $x$ and variance 1.0. So, with very high probability, any reference attribute generated from e.x will be in the range $[e . x-3, e . x+3]$. So this range in the attribute domain is considered to be 'occupied' by entity $e$. Any entity has an ambiguous attribute if its occupied range intersects with that of another entity. 
Now I come to the generation of co-occurrence relationships from the entity collaboration graph. In this stage, $R$ co-occurrence relationships or hyper-edges are generated, each with its own references. For each hyper-edge $\left\langle r_{i}, r_{i 1}, \ldots, r_{i k}\right\rangle$, two aspects need to be controlled — how many references and which references should be included in this hyper-edge. This is done as follows. First, I sample an entity $e_{i}$ which serves the initiator entity for this hyper-edge. Then other entities $e_{i j}$ for this hyper-edge are repeatedly sampled (without replacement) from the neighbors of the initiator entity $e_{i}$. The size of the hyper-edge is determined using a parameter $p_{c}$. The sampling step for a hyper-edge is terminated with probability $p_{c}$ after each selection $e_{i j}$. The process is also terminated when the neighbors of the initiator entity are exhausted. Finally, references $r_{i j}$ need to be generated from each of the selected entities $e_{i j}$. This is done for each entity $e$ by sampling from its Gaussian distribution $\mathcal{N}(e . x, 1)$.

As we have seen, the data generation process controls different aspects of the data that are useful for analyzing the models and algorithms proposed in this dissertation. The synthetic data generation model is motivated by the domain of academic collaborations, but is general enough to handle different kinds of relational data. Essentially, it models any domain that has entities with potentially ambiguous attributes, relationships between those entities, and hyper-edges of arbitrary arity that contain noisy references to these entities. So this model can mimic different kinds of noisy relational data, for example, email data, where names of different people get mentioned in the same email, and product shopping data with noisy descriptions of different products that people may buy together. In effect, this data 
generator enables us to investigate the impact of collective relational approaches to clustering in domains other than bibliographic data, and also for applications beyond entity resolution. 


\section{Bibliography}

[1] Lada Adamic and Eytan Adar. Friends and neighbors on the web. Social Networks, 25(3):211-230, July 2003.

[2] Eneko Agirre, Jordi Atserias, Lluis Padro, and German Rigau. Combining supervised and unsupervised lexical knowledge methods for word sense disambiguation computers and the humanities. In Computers and the Humanities, Special Double Issue on SensEval. Eds. Martha Palmer and Adam Kilgarriff. 34:1,2, 2000.

[3] Rohit Ananthakrishna, Surajit Chaudhuri, and Venkatesh Ganti. Eliminating fuzzy duplicates in data warehouses. In Proceedings of the 28th International Conference on Very Large Databases (VLDB-2002), Hong Kong, China, 2002.

[4] Charles Antoniak. Mixtures of dirichlet processes with applications to bayesian nonparametric problems. The Annals of Statistics, 2:1152-1174, 1974.

[5] Yoshua Bengio and Christopher Kermorvant. Extracting hidden sense probabilities from bitexts. Technical report, TR 1231, Departement d'informatique et recherche operationnelle, Universite de Montreal, 2003.

[6] Omar Benjelloun, Hector Garcia-Molina, Qi Su, and Jennifer Widom. Swoosh: A generic approach to entity resolution. Technical report, Stanford University, March 2005.

[7] Indrajit Bhattacharya and Lise Getoor. Deduplication and group detection using links. In Proceedings of the 10th ACM SIGKDD Workshop on Link Analysis and Group Detection (LinkKDD-04), Seattle, WA, USA, 2004.

[8] Indrajit Bhattacharya and Lise Getoor. Iterative record linkage for cleaning and integration. In SIGMOD 2004 Workshop on Research Issues on Data Mining and Knowledge Discovery, Paris, France, 2004.

[9] Indrajit Bhattacharya and Lise Getoor. Relational clustering for multi-type entity resolution. In The 11th ACM SIGKDD Workshop on Multi Relational Data Mining (MRDM-05), Chicago, IL, USA, 2005.

[10] Indrajit Bhattacharya and Lise Getoor. Collective entity resolution in relational data. IEEE Data Engineering Bulletin, Special Issue on Data Cleaning, pages 4-12, June 2006.

[11] Indrajit Bhattacharya and Lise Getoor. Entity Resolution in Graphs, chapter Mining Graph Data (L. Holder and D. Cook, eds.). Wiley, 2006.

[12] Indrajit Bhattacharya and Lise Getoor. A latent dirichlet model for unsupervised entity resolution. In SIAM Conference on Data Mining (SIAM-SDM), Bethesda, MD, USA, 2006. 
[13] Indrajit Bhattacharya and Lise Getoor. Query-time entity resolution. In $A C M$ Conference on Knowledge Discovery and Data Mining (KDD), Philadelphia, PA, USA, 2006.

[14] Indrajit Bhattacharya, Lise Getoor, and Yoshua Bengio. Unsupervised sense disambiguation using bilingual probabilistic models. In Proceedings of The 42nd Annual Meeting of the Association for Computational Linguistics (ACL), Barcelona, Spain, 2004.

[15] Mikhail Bilenko and Raymond Mooney. Adaptive duplicate detection using learnable string similarity measures. In Proceedings of the Ninth ACM SIGKDD International Conference on Knowledge Discovery and Data Mining (KDD-2003), Washington DC, USA, 2003.

[16] Mikhail Bilenko, Raymond Mooney, William Cohen, Pradeep Ravikumar, and Stephen Fienberg. Adaptive name matching in information integration. IEEE Intelligent Systems, 18(5):16-23, 2003.

[17] Mustafa Bilgic, Louis Licamele, Lise Getoor, and Ben Shneiderman. D-dupe: An interactive tool for entity resolution in social networks. In Visual Analytics Science and Technology (VAST), Baltimore, 2006.

[18] David Blei, Thomas Griffiths, Michael Jordan, and Josh Tenenbaum. Hierarchical topic models and the nested chinese restaurant process. In Advances In Neural Information Processing Systems (NIPS), Vancouver, BC, Canada, 2003.

[19] David Blei and Michael Jordan. Variational methods for the dirichlet process. In International Conference on Machine Learning (ICML), Banff, Alberta, Canada, 2004.

[20] David Blei and John Lafferty. Correlated topic models. In Advances in Neural Information Processing Systems, Vancouver, BC, Canada, 2006.

[21] David Blei, Andrew Ng, and Michael Jordan. Latent dirichlet allocation. Journal of Machine Learning Research, 3:951-991, Jan 2003.

[22] Peter Brown, Stephen Della Pietra, Vincent Della Pietra, and Robert Mercer. Word-sense disambiguation using statistical methods. In Meeting of the Association for Computational Linguistics, pages 264-270, 1991.

[23] Rebecca Bruce and Janyce Wiebe. A new approach to sense identification. In ARPA Workshop on Human Language Technology, 1994.

[24] Claire Cardie and Kiri Wagstaff. Noun phrase coreference as clustering. In Proceedings of the Joint SIGDAT Conference on Empirical Methods in Natural Language Processing and Very Large Corpora (EMNLP), College Park, MD, USA, 1999. 
[25] Soumen Chakrabarti, Byron Dom, and Piotr Indyk. Enhanced hypertext categorization using hyperlinks. In Proceedings of the 1998 ACM SIGMOD International Conference on Management of Data, 1998.

[26] Amit Chandel, P. C. Nagesh, and Sunita Sarawagi. Efficient batch top-k search for dictionary-based entity recognition. In Proceedings of the 22nd International Conference on Data Engineering (ICDE'06), Washington, DC, USA, 2006.

[27] Surajit Chaudhuri, Kris Ganjam, Venkatesh Ganti, and Rajeev Motwani. Robust and efficient fuzzy match for online data cleaning. In Proceedings of the 2003 ACM SIGMOD International Conference on Management of Data, San Diego, CA, USA, 2003.

[28] William Cohen. Data integration using similarity joins and a word-based information representation language. ACM Transactions on Information Systems, 18:288-321, 2000.

[29] William Cohen, Henry Kautz, and David McAllester. Hardening soft information sources. In Proceedings of the Sixth International Conference on Knowledge Discovery and Data Mining (KDD-2000), Boston, MA, USA, 2000.

[30] William Cohen, Pradeep Ravikumar, and Stephen Fienberg. A comparison of string distance metrics for name-matching tasks. In Proceedings of the IJCAI2003 Workshop on Information Integration on the Web, Acapulco, Mexico, 2003.

[31] William Cohen and Jacob Richman. Learning to match and cluster large highdimensional data sets for data integration. In Proceedings of the Eighth ACM SIGKDD International Conference on Knowledge Discovery and Data Mining (KDD-2002), Edmonton, Alberta, CA, 2002.

[32] David Cohn and Thomas Hofmann. The missing link: A probabilistic model of document content and hypertext connectivity. In Advances in Neural Information Processing Systems (NIPS), Vancouver, BC, Canada, 2001.

[33] Ido Dagan. Lexical disambiguation: Sources of information and their statistical realization. In Meeting of the Association for Computational Linguistics, Berkeley, CA, USA, 1991.

[34] Ido Dagan and Alon Itai. Word sense disambiguation using a second language monolingual corpus. Computational Linguistics, 20(4):563-596, 1994.

[35] Hal Daumé and Daniel Marcu. A bayesian model for supervised clustering with the dirichlet process prior. Journal of Machine Learning Research, 6:15511577, Sep 2005. 
[36] A. Dempster, N. Laird, and D. Rubin. Maximum likelihood from incomplete data via the EM algorithm. Journal of the Royal Statistical Society, B 39:1-38, 1977.

[37] Mona Diab. Word Sense Disambiguation Within a Multilingual Framework. PhD thesis, University of Maryland, College Park, 2003.

[38] Mona Diab and Philip Resnik. An unsupervised method for word sense tagging using parallel corpora. In Proceedings of the 40th Anniversary Meeting of the Association for Computational Linguistics (ACL-02), Philadelphia, PA, USA, 2002 .

[39] Christopher Diehl, Lise Getoor, and Galileo Namata. Name reference resolution in organizational email archives. In SIAM Conference on Data Mining (SDM), Bethesda, MD, USA, 2006.

[40] AnHai Doan, Ying Lu, Yoonkyong Lee, and Jiawei Han. Object matching for data integration: A profile-based approach. In Proceedings of the IJCAI Workshop on Information Integration on the Web, Acapulco, MX, 2003.

[41] Xin Dong, Alon Halevy, and Jayant Madhavan. Reference reconciliation in complex information spaces. In ACM SIGMOD International Conference on Management of Data, Baltimore, MD, USA, 2005.

[42] Denise Draper and Steve Hanks. Localized partial evaluation of belief networks. In Proceedings of the Annual Conference on Uncertainty in Artificial Intelligence, Seattle, WA, USA, 1994.

[43] Christiane Fellbaum. WordNet: An Electronic Lexical Database. MIT Press, 1998.

[44] I. Fellegi and A. Sunter. A theory for record linkage. Journal of the American Statistical Association, 64:1183-1210, 1969.

[45] Thomas Ferguson. A bayesian analysis of some nonparametric problems. The Annals of Statistics, 1:209-230, 1973.

[46] Daniela Florescu, Eric Simon, and Dennis Shasha. An extensible framework for data cleaning. In Proceedings of the 16th International Conference on Data Engine ering, San Diego, CA, USA, 2000.

[47] Ariel Fuxman, Elham Fazli, and Rene Miller. Conquer: Efficient management of inconsistent databases. In Proceedings of the ACM SIGMOD International Conference on Management of Data, Baltimore, MD, USA, 2005.

[48] Lise Getoor, Nir Friedman, Daphne Koller, and Ben Taskar. Learning probabilistic models of link structure. Journal of Machine Learning Research, 3:679-707, December 2002. 
[49] C. Lee Giles, Kurt Bollacker, and Steve Lawrence. CiteSeer: An automatic citation indexing system. In Proceedings of the Third ACM Conference on Digital Libraries, Pittsburgh, PA, USA, 1998.

[50] Luis Gravano, Panagiotis Ipeirotis, Nick Koudas, and Divesh Srivastava. Text joins for data cleansing and integration in an rdbms. In 19th IEEE International Conference on Data Engineering, Bangalore, India, 2003.

[51] Thomas Griffiths and Mark Steyvers. Finding scientific topics. In Proceedings of the National Academy of Sciences, volume 101, pages 5228-5235, April 2004.

[52] Mauricio Hernández and Salvatore Stolfo. The merge/purge problem for large databases. In Proceedings of the 1995 ACM SIGMOD International Conference on Management of Data (SIGMOD-95), San Jose, CA, USA, 1995.

[53] Thomas Hofmann. Probabilistic latent semantic analysis. In Proc. of Uncertainty in Artificial Intelligence, UAI'99, Stockholm, Sweden, 1999.

[54] Jeremy Hylton. Identifying and merging related bibliographic records. Master's thesis, Department of Electrical Engineering and Computer Science, MIT, 1996.

[55] Nancy Ide. Cross-lingual sense determination: Can it work? In Computers and the Humanities: Special Issue on Senseval, 34:147-152, 2000.

[56] Nancy Ide and Jean Veronis. Word sense disambiguation: The state of the art. Computational Linguistics, 28(1):1-40, 1998.

[57] Sonia Jain and Radford Neal. A split-merge markov chain monte carlo procedure for the dirichlet process mixture model. Technical report, Dept. of Statistics, University of Toronto, 2000.

[58] Sonia Jain and Radford Neal. Splitting and merging components of a nonconjugate dirichlet process mixture model. Technical report, Dept. of Statistics, University of Toronto, 2005.

[59] Istvan Jonyer, Lawrence Holder, and Diane Cook. Graph-based hierarchical conceptual clustering. Journal of Machine Learning Research, 2(1-2):19-43, 2001.

[60] Dmitri Kalashnikov, Sharad Mehrotra, and Zhaoqi Chen. Exploiting relationships for domain-independent data cleaning. In SIAM International Conference on Data Mining (SIAM SDM), Newport Beach, CA, USA, April 21-23 2005.

[61] Adam Kilgarrif and Joseph Rosenzweig. Framework and results for english senseval. Computers and the Humanities, 34(1):15-48, 2000. 
[62] Jon Kleinberg. Authoritative sources in n hyperlinked environment. Journal of the ACM, 46(5):604-632, 1999.

[63] Jeremy Kubica, Andrew Moore, Jeff Schneider, and Yiming Yang. Stochastic link and group detection. In Proceedings of the Eighteenth National Conference on Artificial Intelligence (AAAI), Edmonton, Alberta, Canada, 2002.

[64] John Lafferty, Andrew McCallum, and Fernando Pereira. Conditional random fields: Probabilistic models for segmenting and labeling sequence data. In 18th International Conference on Machine Learning (ICML), Williams College, MA, USA, 2001.

[65] Steve Lawrence, Kurt Bollacker, and C. Lee Giles. Autonomous citation matching. In Proceedings of the Third International Conference on Autonomous Agents, Seattle, WA, USA, 1999.

[66] Wei Li and Andrew McCallum. Pachinko allocation: Dag-structured mixture models of topic correlations. In International Conference on Machine Learning (ICML), Pittsburgh, PA, USA, 2006.

[67] Xin Li, Paul Morie, and Dan Roth. Semantic integration in text: From ambiguous names to identifiable entities. AI Magazine. Special Issue on Semantic Integration, 2005.

[68] David Liben-Nowell and Jon Kleinberg. The link prediction problem for social networks. In 12th International Conference on Information and Knowledge Management (CIKM), New Orleans, LA, USA, 2003.

[69] Dekang Lin. Word sense disambiguation with a similarity smoothed case library. In Computers and the Humanities: Special Issue on Senseval, 34:147152, 2000.

[70] Kenneth Litkowski. Senseval: The cl research experience. In Computers and the Humanities, 34(1-2), pp. 153-8, 2000.

[71] Andrew McCallum, Andres Corrada-Emmanuel, and Xuerui Wang. Topic and role discovery in social networks. In International Joint Conference on Artificial Intelligence (IJCAI), Edinburgh, Scotland, 2005.

[72] Andrew McCallum, Kamal Nigam, and Lyle Ungar. Efficient clustering of high-dimensional data sets with application to reference matching. In Proceedings of the Sixth International Conference On Knowledge Discovery and Data Mining (KDD-2000), Boston, MA, USA, 2000.

[73] Andrew McCallum and Ben Wellner. Conditional models of identity uncertainty with application to noun coreference. In Advances In Neural Information Processing Systems (NIPS), Vancouver, BC, Canada, 2004. 
[74] Diana McCarthy, Rob Koeling, Julie Weeds, and John Carroll. Finding predominant senses in untagged text. In Proceedings of the 42nd Annual Meeting of the Association for Computational Linguistics, Barcelona, Spain, 2004.

[75] Rada Mihalcea. The role of non-ambiguous words in natural language disambiguation. In Proceedings of the Conference on Recent Advances in Natural Language Processing, Borovetz, Bulgaria, 2003.

[76] Brian Milch, Bhaskara Marthi, David Sontag, Stuart Russell, Daniel L. Ong, and Andrey Kolobov. Blog: Probabilistic models with unknown objects. In International Joint Conference on Artificial Intelligence (IJCAI), Edinburgh, Scotland, 2005.

[77] Thomas Minka. Expectation propagation for approximate bayesian inference. In Proceedings of the 17th Conference in Uncertainty in Artificial Intelligence, 2001.

[78] Alvaro Monge and Charles Elkan. The field matching problem: Algorithms and applications. In Proceedings of the Second International Conference on Knowledge Discovery and Data Mining (KDD-96), Portland, OR, USA, 1996.

[79] Alvaro Monge and Charles Elkan. An efficient domain-independent algorithm for detecting approximately duplicate database records. In Proceedings of the SIGMOD 1997 Workshop on Research Issues on Data Mining and Knowledge Discovery, Tuscon, AZ, USA, 1997.

[80] Gonzalo Navarro. A guided tour to approximate string matching. ACM Computing Surveys, 33(1):31-88, 2001.

[81] Radford Neal. Markov chain sampling methods for dirichlet process mixture models. Journal of Computational and Graphical Statistics, 9:249-265, 2000.

[82] Jennifer Neville, Micah Adler, and David Jensen. Clustering relational data using attribute and link information. In Proceedings of the Text Mining and Link Analysis Workshop, Eighteenth International Joint Conference on Artificial Intelligence (IJCAI), Acapulco, Mexico, 2003.

[83] H. Newcombe, J. Kennedy, S. Axford, and A. James. Automatic linkage of vital records. Science, 130:954-959, 1959.

[84] BBC News. Google 'aids doctors' diagnoses'. http://news.bbc.co.uk/2/hi/health/6132856.stm, 10 November 2006.

[85] Lawrence Page, Sergey Brin, Rajeev Motwani, and Terry Winograd. The pagerank citation ranking: Bringing order to the web. Technical report, Stanford Digital Library Technologies Project, 1998. 
[86] Hanna Pasula, Bhaskara Marthi, Brian Milch, Stuart Russell, and Ilya Shpitser. Identity uncertainty and citation matching. In Advances in Neural Information Processing Systems, Vancouver, BC, Canada, 2003.

[87] Vijayshankar Raman and Joseph M. Hellerstein. Potter's wheel: An interactive data cleaning system. In Proceedings of the 27th International Conference on Very Large Databases (VLDB-2001), Rome, Italy, 2001.

[88] Pradeep Ravikumar and William Cohen. A hierarchical graphical model for record linkage. In Proceedings of the Conference on Uncertainty in Artificial Intelligence (UAI), Banff, Alberta, Canada, July 2004.

[89] Philip Resnik. Using information content to evaluate semantic similarity in a taxonomy. In International Joint Conference on Artificial Intelligence (IJ$C A I)$, Montreal, Quebec, Canada, 1995.

[90] Philip Resnik. Selectional preference and sense disambiguation. In Proceedings of ACL Siglex Workshop on Tagging Text with Lexical Semantics, Why, What and How?, Washington, DC, USA, 1997.

[91] Philip Resnik and David Yarowsky. Distinguishing systems and distinguishing senses: new evaluation methods for word sense disambiguation. Natural Language Engineering, 5(2), 1999.

[92] Eric Ristad and Peter Yianilos. Learning string edit distance. IEEE Transactions on Pattern Analysis and Machine Intelligence, 20(5):522-532, 1998.

[93] Michal Rosen-Zvi, Tom Griffiths, Mark Steyvers, and Padhraic Smyth. The author-topic model for authors and documents. In Proceedings of the Conference on Uncertainty in Artificial Intelligence, Banff, Alberta, Canada, 2004.

[94] Sunita Sarawagi and Anuradha Bhamidipaty. Interactive deduplication using active learning. In Proceedings of the Eighth ACM SIGKDD International Conference on Knowledge Discovery and Data Mining (KDD-2002), Edmonton, Alberta, Canada, 2002.

[95] Hinrich Schutze. Automatic word sense discrimination. Computational Linguistics, 24(1):97-123, 1998.

[96] Vivek Sehgal, Lise Getoor, and Peter Viechnicki. Entity resolution in geospatial data integration. In ACM International Symposium on Advances in Geographic Information Systems, Arlington, VA, USA, 2006.

[97] Jianbo Shi and Jitendra Malik. Normalized cuts and image segmentation. IEEE Transactions on Pattern Analysis and Machine Intelligence, 22(8):888$905,2000$. 
[98] Parag Singla and Pedro Domingos. Multi-relational record linkage. In Proceedings of 3rd Workshop on Multi-Relational Data Mining at ACM SIGKDD, Seattle, WA, USA, 2004.

[99] Ben Taskar, Abbeel Pieter, and Daphne Koller. Discriminative probabilistic models for relational data. In Uncertainty in Artificial Intelligence: Proceedings of the Eighteenth Conference (UAI), San Francisco, CA, USA, 2002.

[100] Sheila Tejada, Craig Knoblock, and Steven Minton. Learning object identification rules for information integration. Information Systems Journal, 26(8):635-656, 2001.

[101] Ben Wellner, Andrew McCallum, Fuchun Peng, and Michael Hay. An integrated, conditional model of information extraction and coreference with application to citation matching. In Conference on Uncertainty in Artificial Intelligence (UAI), Banff, Alberta, Canada, 2004.

[102] William Winkler. The state of record linkage and current research problems. Technical report, Statistical Research Division, U.S. Census Bureau, Washington, DC, 1999.

[103] William Winkler. Methods for record linkage and Bayesian networks. Technical report, Statistical Research Division, U.S. Census Bureau, Washington, DC, 2002.

[104] David Yarowsky. Word-sense disambiguation using statistical models of Roget's categories trained on large corpora. In Proceedings of the International Conference on Computational Linguistics, Nantes, France, 1992.

[105] David Yarowsky. One sense per collocation. In Proceedings os the ARPA Human Language Technology Workshop, Princeton, NJ, USA, 1993.

[106] David Yarowsky. Unsupervised word sense disambiguation rivaling supervised methods. In Meeting of the Association for Computational Linguistics, pages 189-196, Cambridge, MA, USA, 1995. 